\title{
The Third Kind Of Particles
}

\author{
ShaoXu Ren \\ Institute of physical science and Engineering \\ Tongji University, 200092, Shanghai, China \\ Corresponding email: shaoxu-ren@hotmail.com
}

\begin{abstract}
There are two kinds of spin particles in nature, the Boson and the Fermion. Those with integer value spin $0 \hbar, 1 \hbar, 2 \hbar \ldots$ are called Bosons, with half-integer value spin $\hbar / 2,3 \hbar / 2,5 \hbar / 2 \ldots$ are called Fermions. It is well known that everything in the universe is made of Bosons and Fermions.

The spin representations of Boson and the Fermion in conventional quantum mechanics are expressed by Hermitian matrices, which are finite dimensional matrices.

Are there so-called the Third Kind Of Particles (TKP), for an example, whose spin maybe $\hbar / 3, \hbar / 4, \hbar / 5, \hbar / 6 \ldots$, which are neither Bosons nor Fermions?

This article concerns about the possible math figure of TKP. More detailed material related to derivative process and ideas evolvement of TKP are given.
\end{abstract}

\section{keywords}

The Third Kind Of Particles TKP; Boson; Fermion; Hermitian matrices; non-Hermitian matrices; Hermitian self-adjoint; positive definite non-Hermitian self-adjoint; finite dimensional matrices; infinite dimensional matrices

\section{Introduction}

All physical observables of conventional quantum mechanics are Hermitian operators. These Hermitian operators $Z$ are defined in Euclidian Space. They satisfy the so-called Hermiticity relation $Z^{+}=Z$ and have real eigenvalues.

But we know that some non-Hermitian operators could also have real eigenvalues; some operators possessing real eigenvalues might be non-Hermitian operators. The Hermiticity of an operator is only a sufficient condition, which guarantees real eigenvalues, it is not a necessary condition.

In recent years, much intensive research efforts have been made in the field of non-Hermitian Hamiltonians with real eigenvalues by PHHQP cite: [1], International Workshop on Pseudo-Hermitian Hamiltonians in Quantum Physics

This paper focuses on the topic of the construction of non-Hermitian angular momentum:

1) Non-Hermitian orbital angular momentum operator $\mathbb{L}_{j}$ are given, we find the eigenvalues of non-Hermitian orbital angular operator $\mathbb{L}_{3}$ can be nonintegral and the wavefunctions of $\mathbb{L}_{3}$ still remain to be single-values

2) Non-Hermitian spin angular momentum operator $\xi_{j, n}^{( \pm)}(j=1,2,3)$ are given, we find the eigenvalue $n$ of non-Hermitian spin operator $\xi_{j, n}^{( \pm)}$can be those of neither Bosons nor Fermions, such kind of spin particles are called The Third Kind Of Particles, TKP. TKP exist in three system, which are not Anyons. 


\section{Why Does conventional spin angular momentum only...... Conventional Spin Angular Momentum Only Possess Eigenvalues with Integer and Half-integer ?}

a) Using commutation (1) that between angular momentum operator $J^{2}$ and operator $J_{3}$

$$
\left[J^{2}, J_{3}\right]_{-}=0
$$

and two eigen-equations (2),(3)

$$
\begin{aligned}
J^{2}|\mu, \lambda\rangle & =\mu|\mu, \lambda\rangle, & \mu \geq 0 \\
J_{3}|\mu, \lambda\rangle & =\lambda|\mu, \lambda\rangle, & \lambda^{2} \geq 0
\end{aligned}
$$

obtain

$$
\left(J^{2}-J_{3}^{2}\right)|\mu, \lambda\rangle=\left(\mu-\lambda^{2}\right)|\mu, \lambda\rangle
$$

b) As $J^{2}=J_{3}^{2}+J_{1}^{2}+J_{2}^{2}$, another eigen-equation (5) is given as

$$
\left(J_{1}^{2}+J_{2}^{2}\right)|\mu, \lambda\rangle=\left(J^{2}-J_{3}^{2}\right)|\mu, \lambda\rangle=\left(\mu-\lambda^{2}\right)|\mu, \lambda\rangle
$$

Due to $J_{1}^{2}+J_{2}^{2}$ is positive Hermitian operator, (5) implies

$$
\begin{aligned}
\mu-\lambda^{2} & \geqq 0 \\
\text { or } \quad \lambda^{2} & \leqq \mu
\end{aligned}
$$

(7) means So $\lambda^{2}$, or $\lambda$ is restricted by $\mu$ !

or by following two recurrence conditions (12),(15)

or by following two formulas (14),(17)

or by being restricted under conditions (21),(22)

c) Further, there exist a top state $\left|\mu, \lambda_{\max }\right\rangle$ such that it can't be raised, suppose $\lambda=\lambda_{\max }$. that is

$$
J^{+}\left|\mu, \lambda_{\max }\right\rangle=0
$$

then

$$
\begin{aligned}
& J^{-} J^{+}\left|\mu, \lambda_{\max }\right\rangle=0 \\
& \left(J^{2}-J_{3}^{2}-J_{3}\right)\left|\mu, \lambda_{\max }\right\rangle=0 \\
& \left.\underline{\left(\mu-\lambda_{\max }^{2}-\lambda_{\text {max }}\right.}\right)\left|\mu, \lambda_{\text {max }}\right\rangle=0
\end{aligned}
$$

(13) showing that

$$
\underline{\mu=\lambda_{\max }^{2}+\lambda_{\max }}
$$


d) Similar processing, there exist a bottom state $\left.\mu, \lambda_{\min }\right\rangle$ such that it can't be lowered, suppose $\lambda=\lambda_{\min }$. that is

$$
J^{-}\left|\mu, \lambda_{\min }\right\rangle=0
$$

then

$$
\begin{aligned}
& J^{+} J^{-}\left|\mu, \lambda_{\text {min }}\right\rangle=0 \\
& \left(J^{2}-J_{3}^{2}+J_{3}\right)\left|\mu, \lambda_{\min }\right\rangle=0 \\
& \left(\mu-\lambda_{\text {min }}^{2}+\lambda_{\text {min }}\right)\left|\mu, \lambda_{\text {min }}\right\rangle=0
\end{aligned}
$$

(16) showing that

$$
\underline{\mu}=\lambda_{\min }^{2}-\lambda_{\min }
$$

e) From $\star$ (14) and *, (17), get

$$
\underline{\mu=\lambda_{\max }^{2}+\lambda_{\max }=\lambda_{\min }^{2}-\lambda_{\min }}
$$

obtain

$$
\lambda_{\min } \boldsymbol{\nabla}=-\lambda_{\max }
$$

f) There are $k$ states between $\left|\mu, \lambda_{\min }\right\rangle$ and $\left|\mu, \lambda_{\max }\right\rangle$

$$
k=\lambda_{\max }-\lambda_{\min } \boldsymbol{\nabla}=\lambda_{\max }-\left(-\lambda_{\max }\right)=2 \lambda_{\max }
$$

hence the maximum value of angular momentun of particle is

$$
\begin{aligned}
\lambda_{\max } & =k / 2 \\
\text { where } \quad k & =0,1,2,3 \ldots
\end{aligned}
$$

Two important expressions (23) and (24) are given, see below:

g) Substituting (21) into (14), obtain the eigenvalue (23) of $J^{2}$

$$
\mu=\lambda_{\max }^{2}+\lambda_{\max } \star=\underline{\lambda_{\max }}\left(\underline{\lambda_{\max }}+1\right)=\underline{k / 2}(\underline{k / 2}+1)
$$

h) The dimensional formula $D$ of angular momentum is given by (24),

$$
D=2 \lambda_{\max }+1=k+1
$$

Formula (24) is suitable for both orbit and spin.

i) For orbital angular momentum, its eigenvalue state $|\mu, \lambda\rangle$ (3) leads

take

$$
k=0,2,4,6, \ldots
$$

$$
\text { eigenvalue of orbital } \lambda_{\max }=k / 2=0,1,2,3, \ldots \quad \text { integer }
$$

dimensionality of function $D=2 \lambda_{\max }+1=1,3,5,7 \ldots$ 
j) For spin angular momentum, its eigenvalue state $|\mu, \lambda\rangle(3)$, there are two choices of $k$. leads two kinds of spin particle as below

The First Kind of Particles : Boson Particles:

$$
\begin{aligned}
\text { take } & =0,2,4,6, \ldots \\
\text { eigenvalues of spin } \lambda_{\max } & =k / 2=0,1,2,3, \ldots \quad \text { integer } \\
\text { dimensionality of matrix } \quad D & =2 \lambda_{\max }+1=1,3,5,7 \ldots
\end{aligned}
$$

The Second Kind of Particles : Fermion Particles:

take

$$
k=1,3,5,7, \ldots
$$

eigenvalues of $\operatorname{spin} \lambda_{\max }=k / 2=1 / 2,3 / 2,5 / 2,7 / 2, \ldots$ half-integer

dimensionality of matrix $D=2 \lambda_{\max }+1=2,4,6,8, \ldots$

\section{What Will Happen if Condition (6) is Broken ? If Condition (6) Is Broken?}

A) Obviously if: the restriction (6) or equivalent to (9) or (12), (15) on operation $J^{+}|\mu, \lambda\rangle$ and operation $J^{-}|\mu, \lambda\rangle$ are removed, then there will appear infinite eigenvectors $\left|\mu, \lambda_{j}\right\rangle$ of $J_{3}$ :

$$
\begin{aligned}
J_{3}\left|\mu, \lambda_{j}\right\rangle & =\lambda_{j}\left|\mu, \lambda_{j}\right\rangle \\
\text { or } j & =\lambda_{\max } \Rightarrow+\infty \\
j & =\lambda_{\min } \Rightarrow-\infty
\end{aligned}
$$

B) Specially further if: the restriction (6) or (7) is broken, and is changed into the restriction (30) or (31)

$$
\begin{aligned}
& \mu-\lambda^{2}<0 \\
& \text { or } \lambda^{2}>\mu
\end{aligned}
$$

(30) implies: eigenvalues (5) of $J_{1}^{2}+J_{2}^{2}$ should be less than zero, that is

$$
\left(J_{1}^{2}+J_{2}^{2}\right)|\mu, \lambda\rangle=\left(\mu-\lambda^{2}\right)|\mu, \lambda\rangle=(\text { negative eigenvalues })|\mu, \lambda\rangle
$$

(32) shows:

$$
\begin{aligned}
& J_{1}^{2}+J_{2}^{2} \text { is no long a positive definite operator } \\
& \text { Hence } \\
& J_{1} \text { is non-Hermitian operator ! } \\
& J_{2} \text { is non-Hermitian operator ! }
\end{aligned}
$$

C) Formula (34) is one of author's motivation for being engrossed in Non-Hermitian angular momentum and TKP. 


\section{The Flow of This Paper Consists of Three Parts:}

\section{Part 1}

0 Why does ......? ; What Will happen If .....

1 Non-Hermitian Spin Angular Momentum $\overrightarrow{\mathbb{T}}$

2 Non-Hermitian Orbital Angular Momentum $\overrightarrow{\mathbb{L}}$

3 Eigenvalues and Eigenvalues Functions of $\mathbb{L}_{3}$

4 The Recurrence Formulae of Normalized Wavefunctions $\Phi_{m, n}: C_{m} \mathbf{E}_{m, n}$ Part 2

5 Semi-Infinite Dimensional Matrices $\Pi_{j, n}^{( \pm)}$: Spin Hierarchy (SH)

6 Infinite Dimensional Representations $\xi_{j, n}^{( \pm)}$: Chaos Spin Hierarchy (CSH)

7 Spin $0 \hbar$ CSH $\{0,+0,(+)\}, \quad \Omega_{j,+0}$

8 Spin $\hbar / 2$ CSH $\{3 / 4,+1 / 2,(+)\}, \quad \Omega_{j,+1 / 2}$

9 Spin $\hbar / 3$ CSH $\{4 / 9,+1 / 3,(+)\}, \quad \Delta_{j,+1 / 3}$

Part 3

10 Non-Hermitian Momentum $\overrightarrow{\mathbb{P}}$, Phase Factor of Fractional Statistics

11 Conclusion

Appendix : Infinitesimal Rotation of TKP

In chapter $\mathbf{0}$, After reviewing the math picture of angular momentum in conventional quantum mechanics, author postlates the key to TKP is to define the construction of non-Hermitian angular momentum operators and to extend the dimensionality of matrix representations of those operators to semi-infinite dimensional, infinite dimensional space.

The possible math figure of TKP

$J_{1}$ and $J_{2}$ should be infinite (semi-infinite) dimensional non-Hermitian matrices

$J^{2}$ and $J_{3}$ are infinite (semi-infinite) dimensional Hermitian diagonal matrices

Chapter 1 introduces non-Hermitian two dimensions spinor matrices $\overrightarrow{\mathbb{T}}$, which contain one space variable $\varphi$. In space $\alpha=h(\varphi), \overrightarrow{\mathbb{T}}$ are good spin operators. 
In chapter $2, \overrightarrow{\mathbb{T}}$ are applied to construct non-Hermitian orbital angular momentum operator $\mathbb{L}_{j}$ in space $\alpha=\mathrm{h}(\varphi) \mathrm{g}(\theta)$.

Chapter 3 shows that $\mathbb{L}_{3}$ can present nonintegral eigenvalues and the wavefunctions of $\mathbb{L}_{3}$ still remain to be single-values.

In chapter 4, it is marvellously revealed that the recurrence formulae obtained from rising operator $\mathbb{L}_{+}$and lowering operator $\mathbb{L}_{-}$, are no bounded.

The substance from chapter 2 to chapter $\mathbf{4}$ are related to space coordinates.

And the mathematical underpinnings of the next five chapters, chapter 5 to chapter 9, are tightly relevent to matrices whose math elements are pure complex numbers.

Chapter 5 introduces notions, ket vector $|m, n\rangle$ and bra vector $\langle m, n \| \equiv\langle m, n| \alpha$ with $m=0,1,2,3 \ldots$, to construct the bases of linear space, in which semi-infinite dimensional matrix representations $\Pi_{j, n}^{( \pm)}$of orbital angular momentum $\mathbb{L}_{j}$ of TKP are given. $\Pi_{j, n}^{( \pm)}$are called Spin Hierarchy (SH)

Chapter 6 extends the range of quantum number $m$ to be $m=0, \pm 1, \pm 2, \pm 3 \ldots$, then infinite dimensional matrix representations, $\xi_{j, n}^{( \pm)}$are obtained. $\xi_{j, n}^{( \pm)}$are called Chaos Spin Hierarchy (CSH)

The values of quantum number $m$ quoted in chapter 5 and chapter $\mathbf{6}$ is ranging between 0 and $\infty$, as expected in chapter $\mathbf{0}$.

In order to illustrate the character of CSH, three typical spin particles are analysed in Chapter 7, 8, 9.

Chapter 7 is Boson case $\Omega_{j,+0}$ of $\mathbf{C S H}$, where quantum number $n=n_{+0}=0$. $\Omega_{j,+0}$ named Island Operator, has three diagonal blocks in its matrix representations.

Chapter 8 is Fermion case $\Omega_{j,+1 / 2}$ of $\mathbf{C S H}$, where quantum number $n=n_{+1 / 2}=+1 / 2 . \Omega_{j,+1 / 2}$ named Island Operator, has three diagonal blocks in its matrix representations.

In chapter 9, quantum number $n$ is taken to be the greatest non-integer and non-half-integer, $n_{+1 / 3}=+1 / 3$. Use symbol $\Delta_{j,+1 / 3}$, named Ocean Operator, for CSH matrix representations, that has two diagonal blocks.

Chapter 10 studies non-Hermitian momentum $\overrightarrow{\mathbb{P}}$, investigates a special practical applications of gauge invariance in space $\mathrm{f}(r) \mathrm{g}(\theta) \mathrm{h}(\varphi)$. It is argued that the phenomenon of phase factor of fractional statistics could be explained by the concept of TKP which are more physical realistic than Anyons are. 


\section{Non-Hermitian Spin Angular Momentum $\overrightarrow{\mathbb{T}}$}

We start from an example of $\hbar / 2$ spin angular momentum, its spin representation (1) is the well known $2 \times 2$ dimensional matrix. Its three components $S_{1}, S_{2}, S_{3}$ are all Hermitian matrices (2) and their eigenvalues are all $\pm \hbar / 2$.

$$
\begin{gathered}
\vec{S}=\frac{1}{2}\left(\begin{array}{ll}
0 & 1 \\
1 & 0
\end{array}\right), \quad \frac{1}{2}\left(\begin{array}{cc}
0 & -i \\
+i & 0
\end{array}\right), \quad \frac{1}{2}\left(\begin{array}{cc}
1 & 0 \\
0 & -1
\end{array}\right) \\
S_{1}^{+}=S_{1}, \quad S_{2}^{+}=S_{2}, \quad S_{3}^{+}=S_{3}
\end{gathered}
$$

$\vec{S}$ satisfies commutation relations

$$
S_{j} S_{k}-S_{k} S_{j}=i \hbar S_{l}
$$

$j, k, l=1,2,3$ are circulative. For convenience, sometimes we choose $\hbar=1$.

\subsection{Non-Hermitian Spin Angular Momentum $\overrightarrow{\mathbb{T}}$}

Now introduce a set of new operators as following

$$
\overrightarrow{\mathbb{T}}=\frac{1}{2}\left(\begin{array}{cc}
0 & e^{-i \varphi} \\
e^{+i \varphi} & 0
\end{array}\right), \frac{1}{2}\left(\begin{array}{cc}
-i \eta & -i \kappa e^{-i \varphi} \\
i \kappa e^{+i \varphi} & i \eta
\end{array}\right), \frac{1}{2}\left(\begin{array}{cc}
\kappa & \eta e^{-i \varphi} \\
-\eta e^{+i \varphi} & -\kappa
\end{array}\right)
$$

where $\kappa, \eta$ are real numbers and

$$
\mathbb{T}_{1}^{2}=\mathbb{T}_{2}^{2}=\mathbb{T}_{3}^{2}=\frac{1}{4}, \quad \kappa^{2}-\eta^{2}=1
$$

$\mathbb{T}_{1}, \mathbb{T}_{2}, \mathbb{T}_{3}$ obey commutation relation

$$
\overrightarrow{\mathbb{T}} \times \overrightarrow{\mathbb{T}}=i \overrightarrow{\mathbb{T}}
$$

Obviously $\mathbb{T}_{1}$ is a Hermitian matrix, but $\mathbb{T}_{2}$ and $\mathbb{T}_{3}$ are non-Hermitian matrices.

$$
\mathbb{T}_{1}^{+}=\mathbb{T}_{1}, \quad \mathbb{T}_{2}^{+} \neq \mathbb{T}_{2}, \quad \mathbb{T}_{3}^{+} \neq \mathbb{T}_{3}
$$

but the eigenvalues of $\mathbb{T}_{1}, \mathbb{T}_{2}, \mathbb{T}_{3}$ are still real numbers $\pm 1 / 2$.

Note when $\eta$ approaches to zero, $\overrightarrow{\mathbb{T}}(4)$ back to $\vec{S}(2)$

In the following paragraphs, it is shown that the math defect (7) of $\overrightarrow{\mathbb{T}}$ could be corrected from researching the inner product space of spin operator. 


\subsection{Hermitian Self-Adjoint Representation of Operator $Z$ : Hermitian Operator $Z^{+}=Z$}

Frist, we define the inner product space

$$
(f, g) \equiv \int d \tau f^{*} g=(g, f)^{*}
$$

superscript sign $*$ is complex conjugatioin. (8) represents integral for continuous variable, and represents matrix scalar multiplication for discontinuous variable. $f$ and $g$ are the vector functions in inner product space.

Then, adjoint operation representation of an operator $Z$ in inner product space (8) can be defined as the operator $Z^{+}$such that

$$
\left(g, Z^{+} f\right) \equiv(f, Z g)^{*}=(Z g, f)
$$

Where

$$
\begin{aligned}
\left(g, Z^{+} f\right) & \equiv(f, Z g)^{*} \quad \Leftrightarrow \quad Z^{+} \equiv\left(Z^{*}\right)^{\sim} \\
(f, Z g)^{*} & =(Z g, f)
\end{aligned}
$$

$\sim$ denotes transpose of a matrix $Z$. If the right side of (9) satisfies

$$
(Z g, f)=(g, Z f)
$$

then (9) becomes

$$
\left(g, Z^{+} f\right) \equiv(f, Z g)^{*}=(Z g, f)=(g, Z f)
$$

we get operator relation

$$
Z^{+}=Z
$$

In case of (12), operator $Z$ is said to be "Hermitian self-adjoint", or to be "self-adjoint" or "Hermitian". As yet all the operators of conventional quantum mechanics are postulated to be Hermitian operators. Sometimes, space (8) is called Hermitian Space. Operator $Z$ satisfying formula (9) is said to be "positive definite operator" in inner product space (8). 


\subsection{Positive definite non-Hermitian Self-Adjoint Representation of Operator Z: Non-Hermitian self-adjoint Operator $Z^{\oplus}=Z$}

Extending the definition (8) of the inner product space to space (13)

$$
(\alpha f, g) \equiv \int d \tau(\alpha f)^{*} g=(g, \alpha f)^{*}
$$

Here $\alpha$ is a metric coefficient operator. $\alpha^{+}=\alpha, \alpha$ is introduced to be the sign of the curve of space. when $\alpha \rightarrow 1,(13) \rightarrow(8)$, space is flat.

Hermitian Space (10) is flat space; when $\alpha \neq 1$, space becomes bent and warped.

Positive Definite Non-Hermitian adjoint operation of an operator $Z$ in inner product space (13) is defined by operator $Z^{\oplus}, " \oplus "$ called circled dag, such that

$$
\begin{array}{cc}
\left(\alpha g, Z^{\oplus} f\right)=(\alpha f, Z g)^{*} \\
\text { If } & Z^{\oplus}=Z
\end{array}
$$

then the $Z$ is said to be "Positive Definite Non-Hermitian self-adjoint Operator". in case of (15), cite: [2]

Next we are going to seek for the explicit expressions of $Z^{\oplus}$, in the case of $Z$ that are derivative operator (A) and matrix operator (B). 
A) Firstly, turn to the definition (14) of adjoint representation $\partial_{x}^{\oplus}$ of a derivative operator $\partial_{x} \equiv \frac{\partial}{\partial x}$, we have

$$
\int d x(\alpha g)^{*} \partial_{x}^{\oplus} f=\left(\int d x(\alpha f)^{*} \partial_{x} g\right)^{*}
$$

We know what is $\partial_{x}$, but not know what $\partial_{x}^{\oplus}$ means, we want to find out the explicit expression of operator $\partial_{x}^{\oplus}$ in the left side of (16).

From the right side of $(16),(F \equiv \alpha f)$, we have

$$
\begin{aligned}
\left(\int d x(\alpha f)^{*} \partial_{x} g\right)^{*} & =\int d x \alpha f \partial_{x} g^{*}=\int d x F \partial_{x} g^{*}=\left.F g^{*}\right|_{a} ^{b}-\int d x\left(\partial_{x} F\right) g^{*} \\
& =0-\int d x\left(\partial_{x} F\right) g^{*}=-\int d x\left(\partial_{x} \alpha f\right) g^{*}=-\int d x g^{*}\left(\partial_{x} \alpha f\right) \\
& =-\int d x g^{*}\left[\alpha\left(\partial_{x} f\right)+\left(\partial_{x} \alpha\right) f\right]=-\int d x g^{*}\left[\alpha \partial_{x}+\left(\partial_{x} \alpha\right)\right] f
\end{aligned}
$$

From the left side of (16), we have

$$
\begin{aligned}
\int d x(\alpha g)^{*} \partial_{x}^{\oplus} f & =\int d x \alpha^{*} g^{*} \partial_{x}^{\oplus} f=\int d x g^{*}\left(\alpha^{*}\right)^{\sim} \partial_{x}^{\oplus} f \\
& =\int d x g^{*} \alpha^{+} \partial_{x}^{\oplus} f=\int d x g^{*} \alpha \partial_{x}^{\oplus} f
\end{aligned}
$$

Comparing formula (18) with formula (17), we deduce that

$$
\int d x g^{*} \alpha \partial_{x}^{\oplus} f=-\int d x g^{*}\left[\alpha \partial_{x}+\left(\partial_{x} \alpha\right)\right] f
$$

further

$$
\begin{aligned}
\alpha \partial_{x}^{\oplus} & =-\alpha \partial_{x}-\left(\partial_{x} \alpha\right) \\
\partial_{x}^{\oplus} & =-\partial_{x}-\alpha^{-1}\left(\partial_{x} \alpha\right)
\end{aligned}
$$

and

$$
\begin{aligned}
\left(-i \partial_{x}\right)^{\oplus} & =-i \partial_{x}-i \alpha^{-1}\left(\partial_{x} \alpha\right) \\
\left(-i \partial_{x}-i \frac{1}{2} \alpha^{-1} \partial_{x} \alpha\right)^{\oplus} & =-i \partial_{x}-i \frac{1}{2} \alpha^{-1} \partial_{x} \alpha
\end{aligned}
$$

B) Secondly, turn to the definition (14) of adjoint representation $Z^{\oplus}$ of a matrix operator $Z$ in inner product space $(\alpha f, g)$. Postulating

$$
\alpha=\left(\begin{array}{ll}
\alpha_{1} & \alpha_{2} \\
\alpha_{3} & \alpha_{4}
\end{array}\right), \quad \alpha^{+}=\alpha, \quad \text { Det } \alpha=1
$$

then base on $\left(\alpha g, Z^{\oplus} f\right)=(\alpha f, Z g)^{*}(14)$, following we can find the explicit expression of matrix operator $Z^{\oplus}$ : 
From the right side of (14), we have

$$
\begin{aligned}
(\alpha f, Z g)^{*} & =\left[\left(f_{1}, f_{2}\right) *\left(\begin{array}{ll}
\alpha_{1} & \alpha_{2} \\
\alpha_{3} & \alpha_{4}
\end{array}\right)\left(\begin{array}{ll}
z_{1} & z_{2} \\
z_{3} & z_{4}
\end{array}\right)\left(\begin{array}{l}
g_{1} \\
g_{2}
\end{array}\right)\right]^{*} \\
& =\left[\left(g_{1}, g_{2}\right)\left[\left(\begin{array}{ll}
\alpha_{1} & \alpha_{2} \\
\alpha_{3} & \alpha_{4}
\end{array}\right)\left(\begin{array}{ll}
z_{1} & z_{2} \\
z_{3} & z_{4}
\end{array}\right)\right]^{\sim}\left(\begin{array}{l}
f_{1} \\
f_{2}
\end{array}\right)^{*}\right]^{*} \\
& =\left(g_{1}, g_{2}\right)^{*}\left[\left(\begin{array}{ll}
\alpha_{1} & \alpha_{2} \\
\alpha_{3} & \alpha_{4}
\end{array}\right)\left(\begin{array}{ll}
z_{1} & z_{2} \\
z_{3} & z_{4}
\end{array}\right)\right]^{\sim *}\left(\begin{array}{l}
f_{1} \\
f_{2}
\end{array}\right) \\
& =\left(g_{1}, g_{2}\right)^{*}\left[\left(\begin{array}{ll}
\alpha_{1} & \alpha_{2} \\
\alpha_{3} & \alpha_{4}
\end{array}\right)\left(\begin{array}{ll}
z_{1} & z_{2} \\
z_{3} & z_{4}
\end{array}\right)\right]^{+}\left(\begin{array}{l}
f_{1} \\
f_{2}
\end{array}\right)
\end{aligned}
$$

From the left side of (14), we have

$$
\left(\alpha g, Z^{\oplus} f\right)=\left(\begin{array}{ll}
g_{1} & g_{2}
\end{array}\right)^{*}\left(\begin{array}{ll}
\alpha_{1} & \alpha_{2} \\
\alpha_{3} & \alpha_{4}
\end{array}\right)\left(\begin{array}{ll}
z_{1} & z_{2} \\
z_{3} & z_{4}
\end{array}\right)^{\oplus}\left(\begin{array}{l}
f_{1} \\
f_{2}
\end{array}\right)
$$

Comparing formula (26) with formula (25), we deduce that

$$
\begin{aligned}
\left(\begin{array}{ll}
\alpha_{1} & \alpha_{2} \\
\alpha_{3} & \alpha_{4}
\end{array}\right)\left(\begin{array}{ll}
z_{1} & z_{2} \\
z_{3} & z_{4}
\end{array}\right)^{\oplus} & =\left[\left(\begin{array}{ll}
\alpha_{1} & \alpha_{2} \\
\alpha_{3} & \alpha_{4}
\end{array}\right)\left(\begin{array}{ll}
z_{1} & z_{2} \\
z_{3} & z_{4}
\end{array}\right)\right]^{+} \\
& =\left(\begin{array}{ll}
z_{1} & z_{2} \\
z_{3} & z_{4}
\end{array}\right)^{+}\left(\begin{array}{ll}
\alpha_{1} & \alpha_{2} \\
\alpha_{3} & \alpha_{4}
\end{array}\right)^{+}=\left(\begin{array}{ll}
Z_{1} & z_{2} \\
z_{3} & z_{4}
\end{array}\right)^{+}\left(\begin{array}{ll}
\alpha_{1} & \alpha_{2} \\
\alpha_{3} & \alpha_{4}
\end{array}\right)
\end{aligned}
$$

Further obtain

$$
\left(\left(\begin{array}{ll}
Z_{1} & Z_{2} \\
Z_{3} & Z_{4}
\end{array}\right)^{\oplus}=\left(\begin{array}{ll}
\alpha_{1} & \alpha_{2} \\
\alpha_{3} & \alpha_{4}
\end{array}\right)^{-1}\left(\begin{array}{ll}
Z_{1} & Z_{2} \\
z_{3} & z_{4}
\end{array}\right)^{+}\left(\begin{array}{ll}
\alpha_{1} & \alpha_{2} \\
\alpha_{3} & \alpha_{4}
\end{array}\right)\right.
$$

or

$$
Z^{\oplus}=\alpha^{-1} Z^{+} \alpha
$$

\section{Note}

\section{Positive definite non-Hermitian Self-Adjoint Representation $Z^{\oplus}$ of Operator $Z$}

when $Z$ is derivative operator $Z=\partial_{x}$, then $\partial_{x}^{\oplus}=-\partial_{x}-\alpha^{-1}\left(\partial_{x} \alpha\right)$

will be used in constructing non-Hermitian ortital angular momentum operator $\overrightarrow{\mathbb{L}}$ and non-Hermitian momentum operator $\overrightarrow{\mathbb{P}}$

when $Z$ is matrix operator,

then

$$
Z^{\oplus}=\alpha^{-1} Z^{+} \alpha
$$

will be used in constructing non-Hermitian spin operator $\overrightarrow{\mathbb{T}}$, just see next...... 


\subsection{Non-Hermitian Spin Angular Momentum Operator $\overrightarrow{\mathbb{T}}$ Is a Good Operator}

Again pay more attention to the fact: $\mathbb{T}_{1}^{+}=\mathbb{T}_{1}, \quad \mathbb{T}_{2}^{+} \neq \mathbb{T}_{2}, \quad \mathbb{T}_{3}^{+} \neq \mathbb{T}_{3}$

$\mathbb{T}_{1}$ is Hermitian operator, however, $\mathbb{T}_{2}$ and $\mathbb{T}_{3}$ are not, so math symbol Hermitian adjoint "+" is not a good adjoin operator operation for non-Hermitian Spin Angular Momentum Operator $\overrightarrow{\mathbb{T}}$.

Now, instead of Hermitian adjoint operation, dag "+", by a new math symbol adjoint circled dag " $\oplus$ "

$$
\text { "+" (9) } \quad \Rightarrow \quad \text { "๑" (14) }
$$

Hermitian Adjoin $\Rightarrow$ Positive Definite Non-Hermitian Adjoint

Base on the definition formula (29), we try to find out a suitable metric coefficient operator $\alpha$ (24), which can ensure the following math operations

$$
\mathbb{T}_{1}^{\oplus}=\mathbb{T}_{1}, \quad \mathbb{T}_{2}^{\oplus}=\mathbb{T}_{2}, \quad \mathbb{T}_{3}^{\oplus}=\mathbb{T}_{3}
$$

In what follows we are going to show how to approach the goal.

Matrices $\mathbb{T}_{1}, \mathbb{T}_{2}, \mathbb{T}_{3}$ can be expressed by matrices $S_{1}, S_{2}, S_{3}$ as following

$$
\begin{aligned}
\mathbb{T}_{1} & =\cos \varphi S_{1}+\sin \varphi S_{2} \\
\mathbb{T}_{2} & =\kappa\left(\cos \varphi S_{2}-\sin \varphi S_{1}\right)-i \eta S_{3} \\
\mathbb{T}_{3} & =+i \eta\left(\cos \varphi S_{2}-\sin \varphi S_{1}\right)+\kappa S_{3}
\end{aligned}
$$

Firstly, taking the adjoint operator $[\overrightarrow{\mathbb{T}}]^{\oplus}$ of $\overrightarrow{\mathbb{T}}$. we gain

$$
\begin{aligned}
{\left[\mathbb{T}_{1}\right]^{\oplus}=} & \cos \varphi\left[S_{1}\right]^{\oplus}+\sin \varphi\left[S_{2}\right]^{\oplus} \\
= & +\cos \varphi S_{1}+\sin \varphi S_{2}+\cos \varphi\left\{\left[S_{1}\right]^{\oplus}-S_{1}\right\}+\sin \varphi\left\{\left[S_{2}\right]^{\oplus}-S_{2}\right\} \\
= & \mathbb{T}_{1}+\cos \varphi\left\{\left[S_{1}\right]^{\oplus}-S_{1}\right\}+\sin \varphi\left\{\left[S_{2}\right]^{\oplus}-S_{2}\right\} \\
{\left[\mathbb{T}_{2}\right]^{\oplus=} } & +\kappa\left(\cos \varphi\left[S_{2}\right]^{\oplus}-\sin \varphi\left[S_{1}\right]^{\oplus}\right)+i \eta\left[S_{3}\right]^{\oplus} \\
= & \kappa\left(\cos \varphi S_{2}-\sin \varphi S_{1}\right)-i \eta S_{3} \\
& +\kappa\left\{\cos \varphi\left\{\left[S_{2}\right]^{\oplus}-S_{2}\right\}-\sin \varphi\left\{\left[S_{1}\right]^{\oplus}-S_{1}\right\}\right\}+i \eta\left(\left[S_{3}\right]^{\oplus}+S_{3}\right) \\
= & \mathbb{T}_{2}+\kappa\left\{\cos \varphi\left\{\left[S_{2}\right]^{\oplus}-S_{2}\right\}-\sin \varphi\left\{\left[S_{1}\right]^{\oplus}-S_{1}\right\}\right\}+i \eta\left(\left[S_{3}\right]^{\oplus}+S_{3}\right) \\
{\left[\mathbb{T}_{3}\right]^{\oplus=} } & -i \eta\left(\cos \varphi\left[S_{2}\right]^{\oplus}-\sin \varphi\left[S_{1}\right]^{\oplus}\right)+\kappa\left[S_{3}\right]^{\oplus} \\
= & i \eta\left(\cos \varphi S_{2}-\sin \varphi S_{1}\right)+\kappa S_{3} \\
& -i \eta\left\{\cos \varphi\left(\left[S_{2}\right]^{\oplus}+S_{2}\right)-\sin \varphi\left(\left[S_{1}\right]^{\oplus}+S_{1}\right)\right\}+\kappa\left(\left[S_{3}\right]^{\oplus}-S_{3}\right) \\
= & \mathbb{T}_{3}-i \eta\left\{\cos \varphi\left(\left[S_{2}\right]^{\oplus}+S_{2}\right)-\sin \varphi\left(\left[S_{1}\right]^{\oplus}+S_{1}\right)\right\}+\kappa\left(\left[S_{3}\right]^{\oplus}-S_{3}\right)
\end{aligned}
$$


From the above three formulas, it is obviously that if $[\overrightarrow{\mathbb{T}}]^{\oplus}=\overrightarrow{\mathbb{T}}$, the following three formulas must be satisfied

$$
\begin{array}{r}
\cos \varphi\left(\left[S_{1}\right]^{\oplus}-S_{1}\right)+\sin \varphi\left(\left[S_{2}\right]^{\oplus}-S_{2}\right)=0 \\
+\kappa\left\{\cos \varphi\left\{\left[S_{2}\right]^{\oplus}-S_{2}\right\}-\sin \varphi\left\{\left[S_{1}\right]^{\oplus}-S_{1}\right\}\right\}+i \eta\left(\left[S_{3}\right]^{\oplus}+S_{3}\right)=0 \\
-i \eta\left\{\cos \varphi\left(\left[S_{2}\right]^{\oplus}+S_{2}\right)-\sin \varphi\left(\left[S_{1}\right]^{\oplus}+S_{1}\right)\right\}+\kappa\left(\left[S_{3}\right]^{\oplus}-S_{3}\right)=0
\end{array}
$$

Up to now, math expressions (37),(38),(39) are just formalities, we should design a concrete Positive Definite Non-Hermitian Adjoint operation.

After careful exploration, at long last the suitable candidate (24) is found out ! , that could satisfies requirements of (37),(38),(39). we get

$$
\alpha=\left(\begin{array}{ll}
\alpha_{1} & \alpha_{2} \\
\alpha_{3} & \alpha_{4}
\end{array}\right)=\mathrm{h}(\varphi)=\kappa+2 \eta \mathbb{T}_{1}=\left(\begin{array}{cc}
\kappa & \eta e^{-i \varphi} \\
\eta e^{+i \varphi} & \kappa
\end{array}\right)
$$

where

$$
\text { Det } \mathrm{h}(\varphi)=1 . \quad \kappa^{2}-\eta^{2}=1
$$

Applying (1) and the definition (29) of adjoint operator of operator $\vec{Z}=\vec{S}$ in inner product space $(\alpha f, g)$

$$
Z^{\oplus}=\alpha^{-1} Z^{+} \alpha \quad \Rightarrow \quad S^{\oplus}=\alpha^{-1} S^{+} \alpha
$$

after calculation (43)

$$
[\vec{S}]^{\oplus}=\frac{1}{2}[\vec{\sigma}]^{\oplus}=\frac{1}{2} \mathrm{~h}(\varphi)^{-1} \vec{\sigma} \mathrm{h}(\varphi)
$$

then the adjoint operator $S_{j}^{\oplus}$ can be expressed in terms of $S_{j}$ and $\mathbb{T}_{k}$, namely

$$
\begin{array}{llll}
{\left[\sigma_{1}\right]^{\oplus}=\sigma_{1}+4 i \eta \sin \varphi \mathbb{T}_{3}} & \Rightarrow & {\left[S_{1}\right]^{\oplus}=S_{1}+2 i \eta \sin \varphi \mathbb{T}_{3}} \\
{\left[\sigma_{2}\right]^{\oplus}=\sigma_{2}-4 i \eta \cos \varphi \mathbb{T}_{3}} & \Rightarrow & {\left[S_{2}\right]^{\oplus}=S_{2}-2 i \eta \cos \varphi \mathbb{T}_{3}} \\
{\left[\sigma_{3}\right]^{\oplus}=\sigma_{3}+4 i \eta \mathbb{T}_{2}} & \Rightarrow & {\left[S_{3}\right]^{\oplus}=S_{3}+2 i \eta \mathbb{T}_{2}}
\end{array}
$$

Put the above three expressions into (37),(38),(39), and use following formulae (47.1),(47.2),(47.3)

$$
\begin{aligned}
\mathbb{T}_{1} & =+\cos \varphi S_{1}+\sin \varphi S_{2} \\
\kappa \mathbb{T}_{2}+i \eta \mathbb{T}_{3} & =-\sin \varphi S_{1}+\cos \varphi S_{2} \\
\kappa \mathbb{T}_{3}-i \eta \mathbb{T}_{2} & =S_{3}
\end{aligned}
$$


Then, we can obtain following expressions (48),(49),(50), further (37),(38),(39) are verified. see below processing:

$$
\begin{aligned}
& \text { The left side of (37) } \\
= & \cos \varphi\left(\left[S_{1}\right]^{\oplus}-S_{1}\right)+\sin \varphi\left(\left[S_{2}\right]^{\oplus}-S_{2}\right) \\
= & \cos \varphi\left(+2 i \eta \sin \varphi \mathbb{T}_{3}\right)+\sin \varphi\left(-2 i \eta \cos \varphi \mathbb{T}_{3}\right) \\
= & 0 \text { The right side of }(37)
\end{aligned}
$$

The left side of (38)

$$
\begin{aligned}
& =+\kappa\left\{\cos \varphi\left\{\left[S_{2}\right]^{\oplus}-S_{2}\right\}-\sin \varphi\left\{\left[S_{1}\right]^{\oplus}-S_{1}\right\}\right\}+i \eta\left(\left[S_{3}\right]^{\oplus}+S_{3}\right) \\
& =+\kappa\left\{\cos \varphi\left\{-2 i \eta \cos \varphi \mathbb{T}_{3}\right\}-\sin \varphi\left\{+2 i \eta \sin \varphi \mathbb{T}_{3}\right\}\right\}+i \eta\left(2 S_{3}+2 i \eta \mathbb{T}_{2}\right) \\
& =+2 i \eta\left\{-\kappa \mathbb{T}_{3}+S_{3}+i \eta \mathbb{T}_{2}\right\}=0 \text { The right side of (38) }
\end{aligned}
$$

The left side of (39)

$$
\begin{aligned}
& =-i \eta\left\{\cos \varphi\left(\left[S_{2}\right]^{\oplus}+S_{2}\right)-\sin \varphi\left(\left[S_{1}\right]^{\oplus}+S_{1}\right)\right\}+\kappa\left(\left[S_{3}\right]^{\oplus}-S_{3}\right) \\
& =-i \eta\left\{\cos \varphi\left(2 S_{2}-2 i \eta \cos \varphi \mathbb{T}_{3}\right)-\sin \varphi\left(2 S_{1}+2 i \eta \sin \varphi \mathbb{T}_{3}\right\}+\kappa\left(2 i \eta \mathbb{T}_{2}\right)\right. \\
& =-2 i \eta\left\{\cos \varphi S_{2}-\sin \varphi S_{1}-i \eta \mathbb{T}_{3}-\kappa \mathbb{T}_{2}\right\}=0 \text { The right side of (39) }
\end{aligned}
$$

The above three formulae could ensure $\mathbb{T}_{1}^{\oplus}(34), \mathbb{T}_{2}^{\oplus}(35), \mathbb{T}_{3}^{\oplus}(36)$ to equal to $\mathbb{T}_{1}, \mathbb{T}_{2}, \mathbb{T}_{3}, \quad\left[\mathbb{T}_{1}^{\oplus}=\mathbb{T}_{1}, \mathbb{T}_{2}^{\oplus}=\mathbb{T}_{2}, \mathbb{T}_{3}^{\oplus}=\mathbb{T}_{3}\right.$ (32) ] or (51)

$$
[\overrightarrow{\mathbb{T}}]^{\oplus}=\overrightarrow{\mathbb{T}}
$$

Next, using (29), we can again directly prove (51).

$$
\begin{aligned}
{\left[\mathbb{T}_{1}\right]^{\oplus} } & =\mathrm{h}^{-1}(\varphi) \mathbb{T}_{1}^{+} \mathrm{h}(\varphi) \\
& \left.=\left(\begin{array}{cc}
\kappa & -\eta e^{-i \varphi} \\
-\eta e^{+i \varphi} & \kappa
\end{array}\right) \frac{1}{2}\left(\begin{array}{cc}
0 & e^{-i \varphi} \\
e^{+i \varphi} & 0
\end{array}\right)\right)^{+}\left(\begin{array}{cc}
\kappa & \eta e^{-i \varphi} \\
\eta e^{+i \varphi} & \kappa
\end{array}\right) \\
& =\frac{1}{2}\left(\begin{array}{cc}
\kappa & -\eta e^{-i \varphi} \\
-\eta e^{+i \varphi} & \kappa
\end{array}\right)\left(\begin{array}{cc}
0 & e^{-i \varphi} \\
e^{+i \varphi} & 0
\end{array}\right)\left(\begin{array}{cc}
\kappa & \eta e^{-i \varphi} \\
\eta e^{+i \varphi} & \kappa
\end{array}\right) \\
& =\frac{1}{2}\left(\begin{array}{cc}
\kappa & -\eta e^{-i \varphi} \\
-\eta e^{+i \varphi} & \kappa
\end{array}\right)\left(\begin{array}{cc}
\eta & \kappa e^{-i \varphi} \\
\kappa e^{+i \varphi} & \eta
\end{array}\right) \\
& =\frac{1}{2}\left(\begin{array}{cc}
0 & \left(\kappa^{2}-\eta^{2}\right) e^{-i \varphi} \\
\left(\kappa^{2}-\eta^{2}\right) e^{+i \varphi} & 0
\end{array}\right)=\frac{1}{2}\left(\begin{array}{cc}
0 & e^{-i \varphi} \\
e^{+i \varphi} & 0
\end{array}\right)=\mathbb{T}_{1}
\end{aligned}
$$




$$
\begin{aligned}
& {\left[\mathbb{T}_{2}\right]^{\oplus}=\mathrm{h}^{-1}(\varphi) \mathbb{T}_{2}^{+} \mathrm{h}(\varphi)} \\
& =\left(\begin{array}{cc}
\kappa & -\eta e^{-i \varphi} \\
-\eta e^{+i \varphi} & \kappa
\end{array}\right) \frac{1}{2}\left(\begin{array}{cc}
-i \eta & -i \kappa e^{-i \varphi} \\
+i \kappa e^{+i \varphi} & +i \eta
\end{array}\right)^{+}\left(\begin{array}{cc}
\kappa & \eta e^{-i \varphi} \\
\eta e^{+i \varphi} & \kappa
\end{array}\right) \\
& \frac{1}{2}\left(\begin{array}{cc}
\kappa & -\eta e^{-i \varphi} \\
-\eta e^{+i \varphi} & \kappa
\end{array}\right)\left(\begin{array}{cc}
+i \eta & -i \kappa e^{-i \varphi} \\
+i \kappa e^{+i \varphi} & -i \eta
\end{array}\right)\left(\begin{array}{cc}
\kappa & \eta e^{-i \varphi} \\
\eta e^{+i \varphi} & \kappa
\end{array}\right) \\
& =\frac{1}{2}\left(\begin{array}{cc}
\kappa & -\eta e^{-i \varphi} \\
-\eta e^{+i \varphi} & \kappa
\end{array}\right)\left(\begin{array}{cc}
0 & -i\left(\kappa^{2}-\eta^{2}\right) e^{-i \varphi} \\
i\left(\kappa^{2}-\eta^{2}\right) e^{+i \varphi} & 0
\end{array}\right) \\
& =\frac{1}{2}\left(\begin{array}{cc}
\kappa & -\eta e^{-i \varphi} \\
-\eta e^{+i \varphi} & \kappa
\end{array}\right)\left(\begin{array}{cc}
0 & -i e^{-i \varphi} \\
i e^{+i \varphi} & 0
\end{array}\right) \\
& =\frac{1}{2}\left(\begin{array}{cc}
-i \eta & -i \kappa e^{-i \varphi} \\
+i \kappa e^{+i \varphi} & +i \eta
\end{array}\right)=\mathbb{T}_{2} \\
& {\left[\mathbb{T}_{3}\right]^{\oplus}=\mathrm{h}^{-1}(\varphi) \mathbb{T}_{3}^{+} \mathrm{h}(\varphi)} \\
& =\left(\begin{array}{cc}
\kappa & -\eta e^{-i \varphi} \\
-\eta e^{+i \varphi} & \kappa
\end{array}\right) \frac{1}{2}\left(\begin{array}{cc}
\kappa & \eta e^{-i \varphi} \\
-\eta e^{+i \varphi} & -\kappa
\end{array}\right)^{+}\left(\begin{array}{cc}
\kappa & \eta e^{-i \varphi} \\
\eta e^{+i \varphi} & \kappa
\end{array}\right) \\
& =\frac{1}{2}\left(\begin{array}{cc}
\kappa & -\eta e^{-i \varphi} \\
-\eta e^{+i \varphi} & \kappa
\end{array}\right)\left(\begin{array}{cc}
\kappa & -\eta e^{-i \varphi} \\
\eta e^{+i \varphi} & -\kappa
\end{array}\right)\left(\begin{array}{cc}
\kappa & \eta e^{-i \varphi} \\
\eta e^{+i \varphi} & \kappa
\end{array}\right) \\
& =\frac{1}{2}\left(\begin{array}{cc}
\kappa & -\eta e^{-i \varphi} \\
-\eta e^{+i \varphi} & \kappa
\end{array}\right)\left(\begin{array}{cc}
\kappa^{2}-\eta^{2} & 0 \\
0 & -\left(\kappa^{2}-\eta^{2}\right)
\end{array}\right) \\
& =\frac{1}{2}\left(\begin{array}{cc}
\kappa & -\eta e^{-i \varphi} \\
-\eta e^{+i \varphi} & \kappa
\end{array}\right)\left(\begin{array}{cc}
1 & 0 \\
0 & -1
\end{array}\right) \\
& =\frac{1}{2}\left(\begin{array}{cc}
\kappa & \eta e^{-i \varphi} \\
-\eta e^{+i \varphi} & -\kappa
\end{array}\right)=\mathbb{T}_{3}
\end{aligned}
$$

Formulae (52), (53) and (54) show

$$
[\overrightarrow{\mathbb{T}}]^{\oplus}=\mathrm{h}^{-1}(\varphi) \overrightarrow{\mathbb{T}}^{+} \mathrm{h}(\varphi)=\overrightarrow{\mathbb{T}}
$$

In the new space $\alpha=\mathrm{h}(\varphi)$ (40), $\vec{S}$ becomes a non-positive definite non-Hermitian operator, but $\overrightarrow{\mathbb{T}}$ is a positive definite non-Hermitian operator, which are good angular momentum operators, which contain one variable $\varphi$.

\begin{tabular}{|cccccc|}
\hline Note & Space Curvature $\alpha$ \\
\multicolumn{1}{c}{$\alpha=1$} & $\alpha=\mathrm{h}(\varphi)=\kappa+2 \eta \mathbb{T}_{1}$ & \\
{$\left[S_{1}\right]^{+}=S_{1}$} & $\Rightarrow\left[S_{1}\right]^{\oplus} \neq S_{1} \Rightarrow$ & $\left.\Rightarrow \mathbb{T}_{1}\right]^{\oplus}=\mathbb{T}_{1}$ & $(56.1)$ \\
{$\left[S_{2}\right]^{+}=S_{2}$} & $\Rightarrow\left[S_{2}\right]^{\oplus} \neq S_{2} \Rightarrow$ & {$\left[\mathbb{T}_{2}\right]^{\oplus}=\mathbb{T}_{2}$} \\
{$\left[S_{3}\right]^{+}=S_{3}$} & $\Rightarrow\left[S_{3}\right]^{\oplus} \neq S_{3} \Rightarrow$ & {$\left[\mathbb{T}_{3}\right]^{\oplus}=\mathbb{T}_{3}$}
\end{tabular}




\section{Non-Hermitian Orbital Angular Momentum $\overrightarrow{\mathbb{L}}$}

2.1 Hermitian orbital angular momentum are expressed by

$$
\begin{aligned}
& l_{1}=+i \sin \varphi \partial_{\theta}-\cot \theta \cos \varphi l_{3} \\
& l_{2}=-i \cos \varphi \partial_{\theta}-\cot \theta \sin \varphi l_{3} \\
& l_{2}=-i \cos \varphi \partial_{\theta}-\cot \theta \sin \varphi l_{3}
\end{aligned}
$$

they satisfy

$$
l_{1}^{+}=l_{1}, \quad l_{2}^{+}=l_{2}, \quad l_{3}^{+}=l_{3}
$$

Now we extend the definition of metric curvature $\alpha$ from one coordinate function $\mathrm{h}(\varphi)(1-40)$ to two coordinate functions $\mathrm{h}(\varphi) \mathrm{g}(\theta)(5)$. Choose $\alpha$ to be the metric curvature of $\varphi$ and $\theta$ space, given by

$$
\alpha=\mathrm{h}(\varphi) \mathrm{g}(\theta) ; \quad \mathrm{h}(\varphi)=\kappa+2 \eta \mathbb{T}_{1}, \quad \mathrm{~g}(\theta)=\sin ^{1+4 m_{0}} \theta
$$

In space $\alpha$ (5), by means of $\partial_{x}^{\oplus}=-\partial_{x}-\alpha^{-1}\left(\partial_{x} \alpha\right)$ (1-21), we have

$$
\begin{aligned}
\partial_{\varphi}^{\oplus}= & -\partial_{\varphi}-(\mathrm{h}(\varphi))^{-1}\left(\partial_{\varphi} \mathrm{h}(\varphi)\right)=-\partial_{\varphi}-2 \eta \mathbb{T}_{2} \\
\partial_{\theta}^{\oplus}= & -\partial_{\theta}-(\mathrm{g}(\theta))^{-1}\left(\partial_{\theta} \mathrm{g}(\theta)\right)=-\partial_{\theta}-\left(1+4 m_{0}\right) \cot \theta \\
& l_{1}^{\oplus}=l_{1}+i 4 m_{0} \sin \varphi \cot \theta+2 i \eta \cot \theta \cos \varphi \mathbb{T}_{2} \\
& l_{2}^{\oplus}=l_{2}-i 4 m_{0} \cos \varphi \cot \theta+2 i \eta \cot \theta \sin \varphi \mathbb{T}_{2} \\
& l_{3}^{\oplus}=l_{3}-i 2 \eta \mathbb{T}_{2}
\end{aligned}
$$

$$
\text { Note } \quad l_{1}^{\oplus} \neq l_{1}, \quad l_{2}^{\oplus} \neq l_{2}, \quad l_{3}^{\oplus} \neq l_{3}
$$

Now in space (5) $\alpha=\mathrm{h}(\varphi) \mathrm{g}(\theta) ; \mathrm{h}(\varphi)=\kappa+2 \eta \mathbb{T}_{1}, \mathrm{~g}(\theta)=\sin ^{1+4 m_{0}} \theta$

$$
\text { define new operators } \quad \overrightarrow{\mathbb{L}} \equiv \frac{1}{2}\left\{\vec{l}^{\oplus}+\vec{l}\right)
$$

further obtain

$$
\begin{aligned}
& \mathbb{L}_{1}=+i \sin \varphi\left(\partial_{\theta}+2 m_{0} \cot \theta\right)-\cot \theta \cos \varphi \mathbb{L}_{3} \\
& \mathbb{L}_{2}=-i \cos \varphi\left(\partial_{\theta}+2 m_{0} \cot \theta\right)-\cot \theta \sin \varphi \mathbb{L}_{3} \\
& \mathbb{L}_{3}=l_{3}-i \eta \mathbb{T}_{2}=-i \partial_{\varphi}-i \eta \mathbb{T}_{2}
\end{aligned}
$$

Obviously

$$
\mathbb{L}_{1}^{\oplus}=\mathbb{L}_{1}, \quad \mathbb{L}_{2}^{\oplus}=\mathbb{L}_{2}, \quad \mathbb{L}_{3}^{\oplus}=\mathbb{L}_{3}
$$

Further

$\overrightarrow{\mathbb{L}}$ are Positive Definite Non-Hermitiana self-djoint Operators each component of operators $\mathbb{L}_{j}$ includes Hermitian orbital angular momentum $l_{j}$ and some non-Hermitian operators 
It can be shown that non-Hermitian operator $\overrightarrow{\mathbb{L}}$ obeys the angular momentum commutation relation just as the conventional Hermitian orbital angular momentum operator $\vec{l}$ does.

$$
\overrightarrow{\mathbb{L}} \times \overrightarrow{\mathbb{L}}=i \overrightarrow{\mathbb{L}}
$$

Commutation rules (17) shows that non-Hermitian operators, $\mathbb{L}_{1}(13), \mathbb{L}_{2}(14)$, $\mathbb{L}_{3}(15)$ are orbital angular momentum operators.

\subsection{Properties of $\mathbb{L}^{2}, \mathbb{L}_{+}, \mathbb{L}_{-}, \mathbb{L}_{3}$}

Square operator

$$
\begin{aligned}
\overrightarrow{\mathbb{L}}^{2} & =\mathbb{L}_{1}^{2}+\mathbb{L}_{2}^{2}+\mathbb{L}_{3}^{2} \\
& =-\left\{\partial_{\theta}^{2}+\left(1+4 m_{0}\right) \cot \theta \partial_{\theta}-(\sin \theta)^{-2}\left(\mathbb{L}_{3}^{2}-4 m_{0}^{2}\right)-4 m_{0}^{2}-2 m_{0}\right\}
\end{aligned}
$$

Some results about rising operator $\mathbb{L}_{+}$and lowering operator $\mathbb{L}_{-}$:

Using (13),(14) we have

$$
\begin{aligned}
& \mathbb{L}_{+}=\mathbb{L}_{1}+i \mathbb{L}_{2}=e^{+i \varphi}\left\{+\partial_{\theta}-\cot \theta\left(\mathbb{L}_{3}-2 m_{0}\right)\right\} \\
& \mathbb{L}_{-}=\mathbb{L}_{1}-i \mathbb{L}_{2}=e^{-i \varphi}\left\{-\partial_{\theta}-\cot \theta\left(\mathbb{L}_{3}+2 m_{0}\right)\right\}
\end{aligned}
$$

The following formulae ban be expressed by (15),(19) and (20),(21)

$$
\begin{gathered}
\mathbb{L}_{+} \mathbb{L}_{-}=\mathbb{L}^{2}-\mathbb{L}_{3}^{2}-\mathbb{L}_{3} \\
\mathbb{L}_{-} \mathbb{L}_{+}=\mathbb{L}^{2}-\mathbb{L}_{3}^{2}+\mathbb{L}_{3} \\
\mathbb{L}_{+} \mathbb{L}_{-}-\mathbb{L}_{-} \mathbb{L}_{+}=2 \mathbb{L}_{3} \\
\mathbb{L}_{+} \mathbb{L}_{-}+\mathbb{L}_{-} \mathbb{L}_{+}=2\left\{\mathbb{L}^{2}-\mathbb{L}_{3}^{2}\right\} \\
{\left[\mathbb{L}_{3}, \mathbb{L}_{+}\right]_{-}=+\mathbb{L}_{+}} \\
{\left[\mathbb{L}_{3}, \mathbb{L}_{+}\right]_{-}=+\mathbb{L}_{+}} \\
\mathbb{L}^{2}=\frac{1}{2}\left(\mathbb{L}_{+} \mathbb{L}_{-}+\mathbb{L}_{-} \mathbb{L}_{+}\right)+\mathbb{L}_{3}^{2} \\
\mathbb{L}_{1}^{2}+\mathbb{L}_{2}^{2}=\frac{1}{2}\left(\mathbb{L}_{+} \mathbb{L}_{-}+\mathbb{L}_{-} \mathbb{L}_{+}\right) \\
+\mathbb{L}_{-} \mathbb{L}_{+}=2\left\{\mathbb{L}^{2}-\mathbb{L}_{3}^{2}\right\} \\
\mathbb{L}_{1}=\frac{1}{2}\left\{\mathbb{L}_{+}+\mathbb{L}_{-}\right\} \\
\mathbb{L}_{2}=\frac{1}{2 \mathrm{i}}\left\{\mathbb{L}_{+}-\mathbb{L}_{-}\right\}
\end{gathered}
$$

(28) and (22) (23) show

$$
\left[\mathbb{L}^{2}, \mathbb{L}_{3}\right]_{-}=0
$$

(33) shows $\mathbb{L}^{2}$ and $\mathbb{L}_{3}$ have common eigenfunction. 


\section{Eigenvalues and}

\section{Eigenvalues Functions of $\mathbb{L}_{3}$}

3.1 Now let us turn to discuss eigenvalues of non-Hermitian operator $\mathbb{L}_{3}$

$$
\begin{aligned}
\mathbb{L}_{3} & =l_{3}-i \eta \mathbb{T}_{2}=l_{3}-\frac{1}{2} i \eta\left[\begin{array}{cc}
-i \eta & -i \kappa e^{-i \varphi} \\
i \kappa e^{+i \varphi} & i \eta
\end{array}\right] \\
& =\left[\begin{array}{cc}
l_{3}-\frac{1}{2} \eta^{2} & -\frac{1}{2} \eta \kappa e^{-i m \varphi} \\
+\frac{1}{2} \eta \kappa e^{+i m \varphi} & l_{3}+\frac{1}{2} \eta^{2}
\end{array}\right]
\end{aligned}
$$

Operator $\mathbb{L}_{3}$ (1) yields two groups of eigenvalue functions, $\phi_{m}^{+2 m_{0}}$ and $\phi_{m}^{-2 m_{0}}$. Starting with $\phi_{m}^{+2 m_{0}}$

$$
\begin{gathered}
\mathbb{L}_{3} \phi_{m}^{+2 m_{0}}=\mu \phi_{m}^{+2 m_{0}}=0 \\
{\left[\begin{array}{cc}
m-1-\frac{1}{2} \eta^{2}-\mu & -\frac{1}{2} \eta \kappa e^{-i \varphi} \\
+\frac{1}{2} \eta \kappa e^{+i \varphi} & m+\frac{1}{2} \eta^{2}-\mu
\end{array}\right]\left[\begin{array}{c}
D_{1} e^{i(m-1) \varphi} \\
D_{2} e^{i m \varphi}
\end{array}\right]=0}
\end{gathered}
$$

Equivalently determinant Det

$$
\left|\begin{array}{cc}
m-1-\frac{1}{2} \eta^{2}-\mu & -\frac{1}{2} \eta \kappa \\
+\frac{1}{2} \eta \kappa & m+\frac{1}{2} \eta^{2}-\mu
\end{array}\right|=0
$$

Evaluating the determinant of (4)

$$
\begin{aligned}
\text { Det } & =\left(m-\mu-\frac{1}{2} \eta^{2}-1\right)\left(m-\mu+\frac{1}{2} \eta^{2}\right)+\frac{1}{4} \eta^{2} \kappa^{2} \\
& =\left(m-\mu-\frac{1}{2} \eta^{2}\right)\left(m-\mu+\frac{1}{2} \eta^{2}\right)-\left(m-\mu+\frac{1}{2} \eta^{2}\right)+\frac{1}{4} \eta^{2} \kappa^{2} \\
& =(m-\mu)^{2}-\frac{1}{4} \eta^{4}-(m-\mu)-\frac{1}{2} \eta^{2}+\frac{1}{4} \eta^{2} \kappa^{2} \\
& =(m-\mu)^{2}-(m-\mu)-\frac{1}{2} \eta^{2}+\frac{1}{4} \eta^{2}\left(\kappa^{2}-\eta^{2}\right) \\
& =(m-\mu)^{2}-(m-\mu)-\frac{1}{4} \eta^{2}=0
\end{aligned}
$$

The solution of quadratic equation of the determinant (5) is given as

$$
m-\mu=\frac{1}{2}\left(+1 \pm \sqrt{1+\eta^{2}}\right)=\frac{1}{2}(+1 \pm \kappa)
$$

we get the eigenvalues of equation (2)

$$
\begin{aligned}
& \mu=\mu_{1}=m-\frac{1}{2}(\kappa+1)=m-2 m_{0} \\
& \mu=\mu_{2}=m+\frac{1}{2}(\kappa-1)=m+2 m_{0}
\end{aligned}
$$

where

$$
\frac{1}{2}(\kappa-1) \equiv 2 m_{0}
$$


Likewise for $\phi_{m}^{-2 m_{0}}$

$$
\mathbb{L}_{3} \phi_{m}^{-2 m_{0}}=\mu \phi_{m}^{-2 m_{0}}
$$

we have

$$
\left[\begin{array}{cc}
m-\frac{1}{2} \eta^{2}-\mu & -\frac{1}{2} \eta \kappa e^{-i \varphi} \\
+\frac{1}{2} \eta \kappa e^{+i \varphi} & m+1+\frac{1}{2} \eta^{2}-\mu
\end{array}\right]\left[\begin{array}{c}
C_{1} e^{i m \varphi} \\
C_{2} e^{i(m+1) \varphi}
\end{array}\right]=0
$$

Equivalently

$$
\left[\begin{array}{cc}
m-\frac{1}{2} \eta^{2}-\mu & -\frac{1}{2} \eta \kappa \\
+\frac{1}{2} \eta \kappa & m+1+\frac{1}{2} \eta^{2}-\mu
\end{array}\right]\left[\begin{array}{l}
C_{1} \\
C_{2}
\end{array}\right]=0
$$

Evaluating the determinant of (12)

$$
\begin{aligned}
\text { Det } & =\left(m-\mu-\frac{1}{2} \eta^{2}\right)\left(m-\mu+\frac{1}{2} \eta^{2}+1\right)+\frac{1}{4} \eta^{2} \kappa^{2} \\
& =(m-\mu)^{2}+(m-\mu)-\frac{1}{4} \eta^{2}=0
\end{aligned}
$$

The solution of quadratic equation of the determinant (13) is given as

$$
m-\mu=\frac{1}{2}\left(-1 \pm \sqrt{1+\eta^{2}}\right)=\frac{1}{2}(-1 \pm \kappa)
$$

we get the eigenvalues of equation (10)

$$
\begin{aligned}
& \mu=\mu_{3}=m-\frac{1}{2}(\kappa-1) \\
& \mu=\mu_{4}=m+\frac{1}{2}(\kappa+1)
\end{aligned}
$$

Because when $\eta$ approaches to zero $(\kappa=1), \mathbb{L}_{3}$ equals to $l_{3}$, the eigenvalues of $\mathbb{L}_{3}$ and the eigenvalues $l_{3}$ should be the same. So the reasonable solutions are $\mu_{2}$ and $\mu_{3}$.

$$
\begin{aligned}
& \text { for } \phi_{m}^{+2 m_{0}} \text { (2): } \mu_{+}=\mu_{2}=m+\frac{1}{2}(\kappa-1)=m+2 m_{0} \\
& \text { for } \phi_{m}^{-2 m_{0}}(10): \quad \mu_{-}=\mu_{3}=m-\frac{1}{2}(\kappa-1)=m-2 m_{0} \\
& \mathbb{L}_{3} \phi_{m}^{+2 m_{0}}=\mu_{+} \phi_{m}^{+2 m_{0}}=\left(m+2 m_{0}\right) \phi_{m}^{+2 m_{0}} \\
& \mathbb{L}_{3} \phi_{m}^{-2 m_{0}}=\mu_{-} \phi_{m}^{-2 m_{0}}=\left(m-2 m_{0}\right) \phi_{m}^{-2 m_{0}}
\end{aligned}
$$


normalized functions

$$
\begin{aligned}
& \phi_{m}^{+2 m_{0}}=\frac{1}{\sqrt{4 \pi}}\left[\begin{array}{c}
-\sqrt{\kappa-1} e^{i(m-1) \varphi} \\
+\sqrt{\kappa+1} e^{i m \varphi}
\end{array}\right] \\
& \phi_{m}^{-2 m_{0}}=\frac{1}{\sqrt{4 \pi}}\left[\begin{array}{c}
+\sqrt{\kappa+1} e^{i m \varphi} \\
-\sqrt{\kappa-1} e^{i(m+1) \varphi}
\end{array}\right]
\end{aligned}
$$

Orthogonality-normalization integrals are given as follows

$$
\begin{aligned}
& \int_{0}^{2 \pi} d \varphi\left(\phi_{m_{1}}^{ \pm 2 m_{0}}\right) * \mathrm{~h}(\varphi) \phi_{m_{2}}^{ \pm 2 m_{0}}=\delta_{m_{1}, m_{2}} \\
& \int_{0}^{2 \pi} d \varphi\left(\phi_{m_{1}}^{ \pm 2 m_{0}}\right) * \mathrm{~h}(\varphi)\left(\phi_{m_{2}}^{\mp 2 m_{0}}\right)^{*}=0
\end{aligned}
$$

3.2 Let us look at two limiting cases of our special interest in (2) and (10) 1) As

$$
\begin{aligned}
2 m_{0} & =0, \quad \mathbb{L}_{3} \Rightarrow l_{3}=-i \partial / \partial_{\varphi} \\
\phi_{m}^{+0} & \approx \phi_{m}^{-0} \Rightarrow \phi_{m}=\frac{1}{\sqrt{2 \pi}} e^{i m \varphi}
\end{aligned}
$$

Non-Hermitian operator $\mathbb{L}_{3}$ backs to Hermitian operator $l_{3}$, and two spinor solutions $\phi_{m}^{+2 m_{0}}, \phi_{m}^{-2 m_{0}}$ degenerate to a scalar solution $\phi_{m}$ of $l_{3}$

2) As

$$
\begin{aligned}
m & =0, \quad \mathbb{L}_{3} \phi_{0}^{ \pm 2 m_{0}}=\mu_{0} \hbar \phi_{0}^{ \pm 2 m_{0}}= \pm 2 m_{0} \hbar \phi_{0}^{ \pm 2 m_{0}} \\
\mu_{0} & = \pm 2 m_{0}
\end{aligned}
$$

$2 m_{0} \hbar$ is just the so-called intrinsic and inherent orbital angular momentum of the quantum particle.

\section{Note}

It should point out that $\mathbb{L}_{3}$ is an non-Hermitian operator, however its eigenvalues $\mu_{ \pm}$(19),(20) can be real numbers.

When $m \pm 2 m_{0}$ is nonintegrals, its eigenfunctions $\phi_{m}^{+2 m_{0}}$ (21) and $\phi_{m}^{-2 m}$

still remain to be single-valuedfunctions!

In conventional quantum mechanics, eigenvalues of orbital angular momentum should be integral numbers, however, eigenvalues of non-Hermitian orbital angular momentum $\mathbb{L}_{3}$ could be nonintegral. 


\section{The Recurrence Formulae of Normalized Wavefunctions $\Phi_{m, n}=C_{m} \mathrm{E}_{m, n}$}

Note

After discussion of eigenvalues and wavefunctions of $\mathbb{L}_{3}$, it is natural to wonder about what will happen ?

if we use the other two non-Hermitian orbital angular momentums

$\mathbb{L}_{1}(2-13), \mathbb{L}_{2}$, (2-14) or their combination,

raising operator $\mathbb{L}_{+}(2-20)$, lowering operator $\mathbb{L}_{-}(2-21)$ to act on two spinor ground state wavefunctions of $\mathbb{L}_{3}, \quad(3-27), \phi_{m=0}^{+2 m_{0}}$, and $\phi_{m=0}^{-2 m_{0}}$

The Recurrence Formulae of $\Phi_{m, n}$, with infinite series, appear ! here $\Phi_{m, n}$ are the common normalized wavefunctions of $\mathbb{L}^{2}$ and $\mathbb{L}_{3}$ (2-33)

\subsection{The Influence of $\mathbb{L}^{2}$ on Eigenfunctions $\phi_{m}^{ \pm 2 m_{0}}$ of $\mathbb{L}_{3}$}

Firistly, consider the eigenvalue equation of $\mathbb{L}^{2}$

$$
\mathbb{L}^{2} \phi_{m}^{ \pm 2 m_{0}}=v \phi_{m}^{ \pm 2 m_{0}}
$$

Using (2-19), the left side of (1) becomes

$$
\begin{aligned}
\mathbb{L}^{2} \phi_{m}^{ \pm 2 m_{0}} & =-\left\{\partial_{\theta}^{2}+\left(1+4 m_{0}\right) \cot \theta \partial_{\theta}-\sin ^{-2} \theta\left(\mathbb{L}_{3}^{2}-4 m_{0}^{2}\right)-4 m_{0}^{2}-2 m_{0}\right\} \phi_{m}^{ \pm 2 m_{0}} \\
& =\left\{0+\sin ^{-2} \theta\left\{\left(m \pm 2 m_{0}\right)^{2}-4 m_{0}^{2}\right\}+4 m_{0}^{2}+2 m_{0}\right\} \phi_{m}^{ \pm 2 m_{0}} \\
& =\left\{\left(m^{2} \pm 4 m m_{0}\right) \sin ^{-2} \theta+4 m_{0}^{2}+2 m_{0}\right\} \phi_{m}^{ \pm 2 m_{0}}
\end{aligned}
$$

The eigenvalue $v$ that in the right side of (1), should be a real constant, so the coefficient $m^{2} \pm 4 m m_{0}$ of function $\sin ^{-2} \theta$ in the right side of (2) must be zero. that is

$$
m^{2} \pm 4 m m_{0}=0
$$

Formula (3) shows: only in the case of quantun number $m=0$, can $2 m_{0}$ remain to be an independent quantun number of quantum number $m$, that is

$$
m=0 \quad \Rightarrow \quad 2 m_{0} \text { be independent }
$$


Hence (2) turns to (6)

$$
\begin{aligned}
& \mathbb{L}_{3} \phi_{0}^{ \pm 2 m_{0}}= \pm 2 m_{0} \phi_{0}^{ \pm 2 m_{0}} \\
& \mathbb{L}^{2} \phi_{0}^{ \pm 2 m_{0}}=2 m_{0}\left(2 m_{0}+1\right) \phi_{0}^{ \pm 2 m_{0}}
\end{aligned}
$$

As a matter of convenience, we introduce the following marks

$$
\begin{aligned}
& n_{+}=+2 m_{0}=t / 2 \\
& n_{-}=-2 m_{0}=s / 2
\end{aligned}
$$

Hence

$$
\begin{aligned}
& \mathbb{L}_{3} \phi_{0}^{ \pm 2 m_{0}}=n_{ \pm} \hbar \phi_{0}^{ \pm 2 m_{0}} \\
& \mathbb{L}^{2} \phi_{0}^{ \pm 2 m_{0}}=n_{ \pm}\left(n_{ \pm} \pm 1\right) \hbar^{2} \phi_{0}^{ \pm 2 m_{0}}
\end{aligned}
$$

4.2 Two Families $\left(\Delta_{m}^{+2 m_{0}}, \Delta_{m}^{-2 m_{0}}\right)$ of Spinor Ground states $\left(\phi_{0}^{+2 m_{0}}, \phi_{0}^{-2 m_{0}}\right)$ of Orbital Angular Momentum $\mathbb{L}_{3}$

From $\mathbb{L}_{+}(2-20), \mathbb{L}_{-}(2-21)$ and $(3-21),(3-22)$ of $\mathbb{L}_{3}$, we have

$$
\begin{aligned}
& \mathbb{L}_{+} \phi_{m}^{ \pm 2 m_{0}}=-\cot \theta\left(m \pm 2 m_{0}-2 m_{0}\right) \phi_{m+1}^{ \pm 2 m_{0}} \\
& \mathbb{L}_{-} \phi_{m}^{ \pm 2 m_{0}}=-\cot \theta\left(m \pm 2 m_{0}+2 m_{0}\right) \phi_{m-1}^{ \pm 2 m_{0}}
\end{aligned}
$$

Next, we analyse the details of (11),(12) carefully. Because of the restriction on quantum number $m(3)$, it is better to start from ground state $\phi_{0}^{ \pm 2 m_{0}}, m=0$ to research the regularity of the action of $\mathbb{L}_{+}$and $\mathbb{L}_{-}$on $\phi_{m}^{ \pm 2 m_{0}}$, hence

For groundstate $\phi_{0}^{+2 m_{0}}$

$$
\begin{aligned}
& \mathbb{L}_{+} \phi_{0}^{+2 m_{0}}=0 \\
& \mathbb{L}_{-} \phi_{0}^{+2 m_{0}}=-4 m_{0} \cot \theta \phi_{-1}^{+2 m_{0}}
\end{aligned}
$$

For groundstate $\phi_{0}^{-2 m_{0}}$

$$
\begin{aligned}
& \mathbb{L}_{+} \phi_{0}^{-2 m_{0}}=+4 m_{0} \cot \theta \phi_{+1}^{-2 m_{0}} \\
& \mathbb{L}_{-} \phi_{0}^{-2 m_{0}}=0
\end{aligned}
$$

Comparison (13),(14) with (15),(16), it ia shown that the effect of $\mathbb{L}_{+}$and $\mathbb{L}_{-}$ on $\phi_{0}^{+2 m_{0}}$ are quite contrary to the effect on $\phi_{0}^{-2 m_{0}}$.

\subsection{Normalized Wavefunctions $\Phi_{m}$ of The Family Members $E_{m}$ of Spinor Ground State Family $\Delta_{m}^{-2 m_{0}}$}

Focus on researching the effect of $\mathbb{L}_{+}$and $\mathbb{L}_{-}$on $\phi_{0}^{-2 m_{0}}\left(n_{-}=n=s / 2\right)$. After a lengthy detailed calculations, we obtain the recurrence formulas below 


$$
\mathrm{E}_{0}=\phi_{0}^{-2 m_{0}}
$$

$$
\begin{aligned}
\mathbb{L}_{+} \mathrm{E}_{0} & =-2 n \mathrm{E}_{1} \\
\mathbb{L}_{-} \mathrm{E}_{0} & =0
\end{aligned}
$$

$\mathrm{E}_{1}=\cot \theta \phi_{+1}^{-2 m_{0}}$

$$
\begin{aligned}
& \mathbb{L}_{+} \mathrm{E}_{1}=\mathrm{E}_{2} \\
& \mathbb{L}_{-} \mathrm{E}_{1}=\mathrm{E}_{0}
\end{aligned}
$$

$$
\begin{array}{r}
\mathrm{E}_{2}=\left\{-(2 n+2) \sin ^{-2} \theta+(2 n+1)\right\} \phi_{+2}^{-2 m_{0}} \\
\mathbb{L}_{+} \mathrm{E}_{2}=+(2 n+2) \mathrm{E}_{3} \\
\mathbb{L}_{-} \mathrm{E}_{2}=-2(2 n+1) \mathrm{E}_{1}
\end{array}
$$

$$
\mathrm{E}_{3}=\left\{(2 n+4) \sin ^{-2} \theta-(2 n+1)\right\} \cot \theta \phi_{+3}^{-2 m_{0}}
$$

$$
\begin{aligned}
& \mathbb{L}_{+} \mathrm{E}_{3}=+\mathrm{E}_{4} \\
& \mathbb{L}_{-} \mathrm{E}_{3}=-3 \mathrm{E}_{2}
\end{aligned}
$$

$$
\mathrm{E}_{4}=\left\{-(2 n+6)(2 n+4) \sin ^{-4} \theta+2(2 n+4)(2 n+3) \sin ^{-2} \theta-(2 n+3)(2 n+1)\right\} \phi_{+4}^{-2 m_{0}}
$$

$$
\begin{aligned}
& \mathbb{L}_{+} \mathrm{E}_{4}=+(2 n+4) \mathrm{E}_{5} \\
& \mathbb{L}_{-} \mathrm{E}_{4}=-4(2 n+3) \mathrm{E}_{3}
\end{aligned}
$$

$$
\mathrm{E}_{5}=\left\{(2 n+8)(2 n+6) \sin ^{-4} \theta-2(2 n+6)(2 n+3) \sin ^{-2} \theta+(2 n+3)(2 n+1)\right\} \cot \theta \phi_{+5}^{-2 m_{0}}
$$

$$
\begin{aligned}
& \mathbb{L}_{+} \mathrm{E}_{5}=(\cdot \cdot) \mathrm{E}_{6} \\
& \mathbb{L}_{-} \mathrm{E}_{5}=-5 \mathrm{E}_{4}
\end{aligned}
$$

Called $E_{m}\left\{E_{0}, E_{1}, E_{2}, \ldots,\right\}$ the family members of ground state $\Delta_{m}^{-2 m_{0}}$

\section{Note}

Unluckily, it seems no hint about the regularity of the recurrence formulas of family members $\left\{E_{0}, E_{1}, E_{2}, \ldots\right\}$ in the above results from (17).till (22)

There must be something ommitted by us. 


\subsection{Normalized Wavefunctions $\Phi_{m}$} as

Normalized wavefunctions $\Phi_{m}$ of the family members $E_{m}$ of $\Delta_{m}^{-2 m_{0}}$ are defined

$$
\begin{aligned}
& \Phi_{m}=C_{m} \mathrm{E}_{m}=C_{m} \xi_{m}(\theta) \phi_{m}^{-2 m_{0}}(\varphi) \\
& \mathrm{E}_{m}=\mathrm{E}_{m}(s)=\mathrm{E}_{m}\left(n \equiv n_{-}=-2 m_{0}\right)=\xi_{m}(\theta) \phi_{m}^{-2 m_{0}}(\varphi) \\
\Delta_{m}^{-2 m_{0}}= & \left\{\mathrm{E}_{0}, \mathrm{E}_{1}, \mathrm{E}_{2}, \ldots \mathrm{E}_{m} \ldots \ldots\right\} \\
= & \left\{\xi_{0}(\theta) \phi_{0}^{-2 m_{0}}(\varphi), \xi_{1}(\theta) \phi_{1}^{-2 m_{0}}(\varphi), \xi_{2}(\theta) \phi_{2}^{-2 m_{0}}(\varphi), \ldots \xi_{m}(\theta) \phi_{m}^{-2 m_{0}}(\varphi) \ldots \ldots\right\}
\end{aligned}
$$

$C_{m}$ are the constants, normalized constant, could be found from the normalization condition (26)

$$
J \equiv \int_{0}^{\pi} d \theta \operatorname{g}(\theta, \lambda) \int_{0}^{2 \pi} d \varphi \Phi_{m}^{+} \mathrm{h}(\varphi, \eta) \Phi_{m}=1
$$

Where $\mathrm{g}(\theta, \lambda)=\sin ^{2 \lambda} \theta \equiv \sin ^{\kappa} \theta, \quad \mathrm{h}(\varphi, \eta)=\kappa+2 \eta \mathbb{T}_{1}$ (2-5) and some marks (27) below

$$
2 \lambda=1+4 m_{0}=\kappa=\sqrt{1+\eta^{2}}=1-2 n=1-s
$$

1) Put $\Phi_{m}(23)$ into $J(26)$, recall (3-23), (26) is simplified as

$$
\begin{aligned}
J & =\left|C_{m}\right|^{2} \int_{0}^{\pi} d \theta \mathrm{g}(\theta) \int_{0}^{2 \pi} d \varphi\left\{\xi_{+m}(\theta) \phi_{m}^{-2 m_{0}}(\varphi)\right\}^{+} \mathrm{h}(\varphi)\left\{\xi_{m}(\theta) \phi_{m}^{-2 m_{0}}(\varphi)\right\} \\
& =\left|C_{m}\right|^{2} \int_{0}^{\pi} d \theta \mathrm{g}(\theta)\left\{\xi_{m}(\theta)\right\}^{2} \int_{0}^{2 \pi} d \varphi\left\{\phi_{m}^{-2 m_{0}}(\varphi)\right\}^{+} \mathrm{h}(\varphi)\left\{\phi_{m}^{-2 m_{0}}(\varphi)\right\} \\
& =\left|C_{m}\right|^{2} \int_{0}^{\pi} d \theta \sin ^{2 \lambda} \theta\left\{\xi_{m}(\theta)\right\}^{2}=1
\end{aligned}
$$

Where

$$
\begin{aligned}
C_{m} & =C_{0 m} / \sqrt{I_{m}} \\
I_{m} & =\int_{0}^{\pi} d \theta \sin ^{2 \lambda} \theta\left\{\xi_{m}(\theta)\right\}^{2}
\end{aligned}
$$


The integrand polynomials $\xi_{m}(\theta)$ come from the families (17), (18), (19), (20), (21), (22)

$$
\begin{aligned}
\xi_{0}(\theta) & =1 \\
\xi_{+1}(\theta) & =\cot \theta \\
\xi_{+2}(\theta) & =-(2 n+2) \sin ^{-2} \theta+(2 n+1) \\
\xi_{+3}(\theta) & =\left\{+(2 n+4) \sin ^{-2} \theta-(2 n+1)\right\} \cot \theta \\
\xi_{+4}(\theta) & =-(2 n+6)(2 n+4) \sin ^{-4} \theta+2(2 n+4)(2 n+3) \sin ^{-2} \theta-(2 n+3)(2 n+1) \\
\xi_{+5}(\theta) & =\left\{(2 n+8)(2 n+6) \sin ^{-4} \theta-2(2 n+6)(2 n+3) \sin ^{-2} \theta+(2 n+3)(2 n+1)\right\} \cot \theta \\
& \quad C_{0 m}= \pm 1, \pm i
\end{aligned}
$$

$C_{0 m}$ is phase factor, has the effect of adjusting $\Phi_{m}$ to the best form that could ensure the recurrence formulas of $\Phi_{m}$ to be the most symmetrical construction.

2) Recall

$$
I(\lambda) \equiv \int_{0}^{\pi} d \theta \sin ^{2 \lambda} \theta=\frac{\pi}{2^{2 \lambda}} \frac{\Gamma(2 \lambda+1)}{\Gamma^{2}(\lambda+1)}
$$

further we obtain the following results:

$$
\begin{aligned}
\text { 1) } \int_{0}^{\pi} \sin ^{2 \lambda} \theta \cdot \cot ^{2} \theta d \theta & =-\frac{1}{2 n} I(\lambda) \\
\text { 2) } \int_{0}^{\pi} \sin ^{2 \lambda} \theta \cdot \sin ^{-2} \theta \cot ^{2} \theta d \theta & =-\frac{1}{2 n} \frac{2 n-1}{2 n+2} I(\lambda) \\
\text { 3) } \int_{0}^{\pi} \sin ^{2 \lambda} \theta \cdot \sin ^{-4} \theta \cot ^{2} \theta d \theta & =-\frac{1}{2 n} \frac{2 n-1}{2 n+2} \frac{2 n+1}{2 n+4} I(\lambda)
\end{aligned}
$$

With the help of the above math preparation, substitute (31), (32), (33), (34), (35), (36) into integral (30), we find

$$
\begin{aligned}
& I_{0}=I(\lambda) \\
& I_{1}=-\frac{1}{2 \mathrm{n}} I(\lambda) \\
& I_{2}=+\frac{2 \mathrm{n}+1}{\mathrm{n}} I(\lambda) \\
& I_{3}=-\frac{3(2 \mathrm{n}+1)}{\mathrm{n}(2 \mathrm{n}+2)} I(\lambda) \\
& I_{4}=+\frac{24(2 \mathrm{n}+3)(2 \mathrm{n}+1)}{2 \mathrm{n}(2 \mathrm{n}+2)} I(\lambda)
\end{aligned}
$$


Further, we get normalized constants $C_{m}$ (29) of integral (30) with subscript index $m=0,1,2,3,4$

$$
\begin{aligned}
C_{0} & =C_{00} / \sqrt{I_{0}}=C_{00} / \sqrt{I(\lambda)} \\
C_{1} & =C_{01} / \sqrt{I_{1}}=C_{01} \sqrt{-2 n} / \sqrt{I(\lambda)}=i C_{01} \sqrt{2 n} / \sqrt{I(\lambda)} \\
C_{2} & =C_{02} / \sqrt{I_{2}}=C_{02} \sqrt{n /(2 n+1)} / \sqrt{I(\lambda)} \\
C_{3} & =C_{03} / \sqrt{I_{3}}=C_{03} \sqrt{-n(2 n+2) / 3(2 n+1)} / \sqrt{I(\lambda)} \\
& =i C_{03} \sqrt{n(2 n+2) / 3(2 n+1)} / \sqrt{I(\lambda)} \\
C_{4} & =C_{04} / \sqrt{I_{4}}=C_{40} \sqrt{2 n(2 n+2) / 24(2 n+1)(2 n+3)} / \sqrt{I(\lambda)}
\end{aligned}
$$

Choosing phase foctors $C_{0 j}$ above as below

$$
C_{00}=+1, \quad C_{01}=+1, \quad C_{02}=+1, \quad C_{03}=-1, \quad C_{04}=-1
$$

Finally, we arrive at the normalized wavefunctions $\Phi_{m}$ of the family members $\mathrm{E}_{m}$ of $\Delta_{m}^{-2 m_{0}}$

$$
\begin{aligned}
\left\{\Phi_{0}, \Phi_{1}, \Phi_{2}, \ldots \Phi_{m}, \ldots\right\}=\left\{C_{0} \mathrm{E}_{0}, C_{1} \mathrm{E}_{1}, C_{2} \mathrm{E}_{2}, \ldots C_{m} \mathrm{E}_{m}, \ldots\right\} \\
\Phi_{0}=+I^{-1 / 2} \cdot \mathrm{E}_{0} \\
\Phi_{1}=+i \sqrt{2 n} I^{-1 / 2} \cdot \mathrm{E}_{1} \\
\Phi_{2}=+\sqrt{2 n / 2 !(2 n+1)} I^{-1 / 2} \cdot \mathrm{E}_{2} \\
\Phi_{3}=-i \sqrt{2 n(2 n+2) / 3 !(2 n+1)} I^{-1 / 2} \cdot \mathrm{E}_{3} \\
\Phi_{4}=-\sqrt{2 n(2 n+2) / 4 !(2 n+1)(2 n+3)} I^{-1 / 2} \cdot \mathrm{E}_{4}
\end{aligned}
$$

They satisfy normalization condition (26). 


\subsection{The Recurrence Formulas of $\Phi_{m}$, resulted from $\mathbb{L}_{+}, \mathbb{L}_{-}$}

Base on normalized wavefunctions $\Phi_{m}(54),(55),(56),(57),(58)$, we spell out the more meaning of the following operators calculation processing

$$
\begin{aligned}
& \mathbb{L}_{+} \Phi_{0}=+I^{-1 / 2}(\lambda) \cdot \mathbb{L}_{+} \mathrm{E}_{0}=+I^{-1 / 2}(\lambda) \cdot-2 n \mathrm{E}_{1} \\
& =+i \sqrt{2 n} \cdot+i \sqrt{2 n} I^{-1 / 2} E_{1} \\
& =\quad+i \sqrt{2 n} \Phi_{1} \\
& \mathbb{L}_{-} \Phi_{0}=+I^{-1 / 2}(\lambda) \cdot \mathbb{L}_{-} \mathrm{E}_{0}=+I^{-1 / 2}(\lambda) \cdot 0 \\
& =0 \\
& \mathbb{L}_{+} \Phi_{1}=+i \sqrt{2 n} I^{-1 / 2}(\lambda) \cdot \mathbb{L}_{+} \mathrm{E}_{1}=+i \sqrt{2 n} I^{-1 / 2}(\lambda) \cdot \mathrm{E}_{2} \\
& =+i \sqrt{2(2 n+1)} \cdot+\sqrt{2 n / 2 !(2 n+1)} I^{-1 / 2} \mathrm{E}_{2} \\
& =+i \sqrt{2(2 n+1)} \Phi_{2} \\
& \mathbb{L}_{-} \Phi_{1}=+i \sqrt{2 n} I^{-1 / 2}(\lambda) \cdot \mathbb{L}_{-} \mathrm{E}_{1}=+i \sqrt{2 n} I^{-1 / 2}(\lambda) \cdot \mathrm{E}_{0} \\
& =+i \sqrt{2 n} \cdot I^{-1 / 2}(\lambda) \mathrm{E}_{0} \\
& =+i \sqrt{2 n} \Phi_{0} \\
& \mathbb{L}_{+} \Phi_{2}=+\sqrt{2 n / 2 !(2 n+1)} I^{-1 / 2} \cdot \mathbb{L}_{+} \mathrm{E}_{2} \\
& =+\sqrt{2 n / 2 !(2 n+1)} I^{-1 / 2} \cdot+(2 n+2) \mathrm{E}_{3} \\
& =+i \sqrt{3(2 n+2)} \cdot-i \sqrt{2 n(2 n+2) / 3 !(2 n+1)} I^{-1 / 2} \mathrm{E}_{3} \\
& =+i \sqrt{3(2 n+2)} \Phi_{3} \\
& \mathbb{L}_{-} \Phi_{2}=+\sqrt{2 n / 2 !(2 n+1)} I^{-1 / 2} \cdot \mathbb{L}_{-} \mathrm{E}_{2} \\
& =+\sqrt{2 n / 2 !(2 n+1)} I^{-1 / 2} \cdot-2(2 n+1) \mathrm{E}_{1} \\
& =\quad+i \sqrt{2(2 n+1)} \cdot+i \sqrt{2 n} I^{-1 / 2} \mathrm{E}_{1}=+i \sqrt{2(2 n+1)} \Phi_{1}
\end{aligned}
$$




$$
\begin{aligned}
& \mathbb{L}_{+} \Phi_{3}=-i \sqrt{2 n(2 n+2) / 3 !(2 n+1)} I^{-1 / 2} \cdot \mathbb{L}_{+} \mathrm{E}_{3} \\
& =-i \sqrt{2 n(2 n+2) / 3 !(2 n+1)} I^{-1 / 2} \cdot \mathrm{E}_{4} \\
& =+i \sqrt{4(2 n+3)} \cdot-\sqrt{2 n(2 n+2) / 4 !(2 n+1)(2 n+3)} I^{-1 / 2} \mathrm{E}_{4} \\
& =+i \sqrt{4(2 n+3)} \Phi_{4} \\
& \mathbb{L}_{-} \Phi_{3}=-i \sqrt{2 n(2 n+2) / 3 !(2 n+1)} I^{-1 / 2} \cdot \mathbb{L}_{-} \mathrm{E}_{3} \\
& =-i \sqrt{2 n(2 n+2) / 3 !(2 n+1)} I^{-1 / 2} \cdot-3 \mathrm{E}_{2} \\
& =+i \sqrt{3(2 n+2)} \cdot+\sqrt{2 n / 2 !(2 n+1)} I^{-1 / 2} \mathrm{E}_{2} \\
& =+i \sqrt{3(2 n+2)} \Phi_{2} \\
& \mathbb{L}_{+} \Phi_{4}=-\sqrt{2 n(2 n+2) / 4 !(2 n+1)(2 n+3)} I^{-1 / 2} \cdot \mathbb{L}_{+} \mathrm{E}_{4} \\
& =-\sqrt{2 n(2 n+2) / 4 !(2 n+1)(2 n+2)} I^{-1 / 2} \cdot+(2 n+4) \mathrm{E}_{5} \\
& =+i \sqrt{5(2 n+4)} \cdot+i \sqrt{2 n(2 n+2)(2 n+4) / 5 !(2 n+1)(2 n+2)} I^{-1 / 2} \mathrm{E}_{5} \\
& =+i \sqrt{5(2 n+4)} \Phi_{5} \\
& \mathbb{L}_{-} \Phi_{4}=-\sqrt{2 n(2 n+2) / 4 !(2 n+1)(2 n+3)} I^{-1 / 2} \cdot \mathbb{L}_{-} \mathrm{E}_{4} \\
& =-\sqrt{2 n(2 n+2) / 4 !(2 n+1)(2 n+3)} I^{-1 / 2} \cdot-4(2 n+3) \mathrm{E}_{3} \\
& =+i \sqrt{4(2 n+3)} \cdot-i \sqrt{2 n(2 n+2) / 3 !(2 n+1)} I^{-1 / 2} \mathrm{E}_{3} \\
& =+i \sqrt{4(2 n+3)} \Phi_{3}
\end{aligned}
$$


Briefly

$$
\begin{array}{ll}
\mathbb{L}_{+} \Phi_{0}=+i \sqrt{1(2 n+0)} \Phi_{1}, & \mathbb{L}_{-} \Phi_{0}=0 \\
\mathbb{L}_{+} \Phi_{1}=+i \sqrt{2(2 n+1)} \Phi_{2}, & \mathbb{L}_{-} \Phi_{1}=+i \sqrt{1(2 n+0)} \Phi_{0} \\
\mathbb{L}_{+} \Phi_{2}=+i \sqrt{3(2 n+2)} \Phi_{3}, & \mathbb{L}_{-} \Phi_{2}=+i \sqrt{2(2 n+1)} \Phi_{1} \\
\mathbb{L}_{+} \Phi_{3}=+i \sqrt{4(2 n+3)} \Phi_{4}, & \mathbb{L}_{-} \Phi_{3}=+i \sqrt{3(2 n+2)} \Phi_{2} \\
\mathbb{L}_{+} \Phi_{4}=+i \sqrt{5(2 n+4)} \Phi_{5}, & \mathbb{L}_{-} \Phi_{4}=+i \sqrt{4(2 n+3)} \Phi_{3}
\end{array}
$$

Obviously! the above results show the regulation of the recurrence formulas of quantum wavefunctions $\Phi_{m}$, the regulation can be extend to the case of $m \Rightarrow \infty$.

By orthogonality-normalization integral (3-23), the normalization condition (26) can further be written into orthogonal-normalization condition (74)

$$
\int_{0}^{\pi} d \theta \mathrm{g}(\theta, \lambda) \int_{0}^{2 \pi} d \varphi \Phi_{k}^{+} \mathrm{h}(\varphi) \Phi_{j}=\delta_{k j}
$$

(54),(55)(56),(57),(58) show: $\Phi_{m}$ is also the function of parameter $n$, introduce vector state $|m, n\rangle$ to represent function $\Phi_{m}=\Phi_{m}(n)$, then

$$
\Phi_{m} \Rightarrow \Phi_{m, n} \equiv|m, n\rangle
$$

Further, the recurrence formulas (69),(70),(71),(72),(73) can be written as the following universal expressions (76) and (77)

$$
\begin{aligned}
& \mathbb{L}_{+}|m, n\rangle=i \sqrt{(m+1)(2 n+m)}|m+1, n\rangle \\
& \mathbb{L}_{-}|m, n\rangle=i \sqrt{m(2 n+m-1)}|m-1, n\rangle
\end{aligned}
$$

where

$$
\begin{aligned}
m & =0,1,2,3, \ldots \ldots \\
2 n & =s=-4 m_{0}
\end{aligned}
$$

The values of $m$ in (78), can be extend to less than zero (80), although (76) and (77) are derived from condition $m=0,1,2,3,4, \ldots \ldots$

Later, we will see in case of (80)

$$
m=0, \pm 1, \pm 2, \pm 3, \ldots \ldots
$$

$\mathbb{L}_{+}$and $\mathbb{L}_{+}$still remain all the properties of angular momentum, and recurrence formulas (76),(77) are still valid. 
Utilize (76),(77), we obtain

$$
\begin{aligned}
\mathbb{L}_{-} \cdot \mathbb{L}_{+}|m, n\rangle & =i \sqrt{(m+1)(2 n+m)} \cdot \mathbb{L}_{-}|m+1, n\rangle \\
& =i \sqrt{(m+1)(2 n+m)} \cdot i \sqrt{(m+1)(2 n+m)}|m, n\rangle \\
& =-(m+1)(2 n+m)|m, n\rangle \\
\mathbb{L}_{+} \cdot \mathbb{L}_{-}|m, n\rangle & =i \sqrt{m(2 n+m-1)} \cdot \mathbb{L}_{+}|m-1, n\rangle \\
& =i \sqrt{m(2 n+m-1)} \cdot i \sqrt{m(2 n+m-1)}|m, n\rangle \\
& =-m(2 n+m-1)|m, n\rangle
\end{aligned}
$$

Then obtain

$$
\begin{aligned}
\left(\mathbb{L}_{+} \mathbb{L}_{-}-\mathbb{L}_{-} \mathbb{L}_{+}\right)|m, n\rangle & =\{-m(2 n+m)+m+m(2 n+m)+2 n+m\}|m, n\rangle \\
& =2(m+n)|m, n\rangle=2 \mathbb{L}_{3}|m, n\rangle \\
\left(\mathbb{L}_{+} \mathbb{L}_{-}+\mathbb{L}_{-} \mathbb{L}_{+}\right)|m, n\rangle & =\{-m(2 n+m)+m-m(2 n+m)-2 n-m\}|m, n\rangle \\
& =\left\{-2 n m-m^{2}+m-2 n m-m^{2}-2 n-m\right\}|m, n\rangle \\
& =2\left\{-2 n m-m^{2}-n^{2}+n^{2}-n\right\}|m, n\rangle \\
& =2\left\{n(n-1)-(m+n)^{2}\right\}|m, n\rangle \\
& =2\left\{n(n-1)-\mathbb{L}_{3}^{2}\right\}|m, n\rangle
\end{aligned}
$$

Recall

$\mathbb{L}_{+} \mathbb{L}_{-}-\mathbb{L}_{-} \mathbb{L}_{+}=2 \mathbb{L}_{3}(2-24)$ and $\mathbb{L}_{+} \mathbb{L}_{-}+\mathbb{L}_{-} \mathbb{L}_{+}=2\left\{\mathbb{L}^{2}-\mathbb{L}_{3}^{2}\right\}(2-25)$

So from (84), we obtain

$$
\mathbb{L}^{2}|m, n\rangle=n(n-1)|m, n\rangle
$$

from (3), (3-20), we get

$$
\mathbb{L}_{3}|m, n\rangle=(m+n)|m, n\rangle
$$

(4-10), is a special case of (85), when for $n_{-}=n$ and $\phi_{0}^{ \pm 2 m_{0}}=|0, n\rangle$

$$
\mathbb{L}^{2}|0, n\rangle=n(n-1)|0, n\rangle
$$

\section{Note}

$$
\text { Formulas (69),(70),(71),(72),(73) }\{(76),(77)\}
$$

are elegance

such kind of recurrence formulas, never have been seen before in the frame of angular momentum theory they should have to bring something unexcepted to physical picture ! 


\section{Semi-Infinite Dimensional Matrices $\Pi_{j}^{( \pm)}$: Spin Hierarchy (SH)}

Representation of Orbital Angular Momentum $\mathbb{L}_{j}$ in linear space $\langle \pm m, n \|$, $| \pm m, n\rangle, \quad(m>0)$

Semi-Infinite Dimensional Matrices $\Pi_{j}^{( \pm)}$are called Spin Hierarchy (SH)

5.1 It will be convenient to use Dirac bra-ket notation to represent the bases of $\alpha$ linear space, when we deal with matrix representations of orbital angular momentum $\mathbb{L}_{j}$.

The bases of $\alpha$ space are marked with the symbols $\langle m, n \|$ and $\mid m, n\rangle$ :

$$
\begin{aligned}
& \text { ket vector (rightvector) }|m, n\rangle \equiv \Phi_{m, n} \\
& \text { bra vector (leftvector) }\left\langle m, n \| \equiv\langle m, n| \alpha=\langle m, n| \mathrm{h}(\varphi) \mathrm{g}(\theta)=\Phi_{m, n} \mathrm{~h}(\varphi) \mathrm{g}(\theta)\right.
\end{aligned}
$$

Then orthogonal-normalization condition (4-74) turns into

$$
\langle\mu, n \| m, n\rangle=\delta_{\mu, m} \equiv \int_{0}^{\pi} d \theta \mathrm{g}(\theta, \lambda) \int_{0}^{2 \pi} d \varphi \Phi_{\mu}^{+} \mathrm{h}(\varphi) \Phi_{m}=\delta_{\mu m}
$$

From (4-76) and (4-77), we have

$$
\begin{aligned}
\left\langle\mu, n \| \mathbb{L}_{+} \mid m, n\right\rangle & =\langle\mu, n \| i \sqrt{(m+1)(2 n+m)} \mid m+1, n\rangle \\
& =i \sqrt{(m+1)(2 n+m)} \delta_{\mu, m+1} \\
\left\langle\mu, n \| \mathbb{L}_{-} \mid m, n\right\rangle & =\langle\mu, n \| i \sqrt{m(2 n+m-1)} \mid m-1, n\rangle \\
& =i \sqrt{m(2 n+m-1)} \delta_{\mu, m-1}
\end{aligned}
$$

After substituting explicit sequence numbers of $\mu$ and $m$ into (4),(5), two series, (4.j) and (5.j) are given

For (4.j)

$$
\begin{aligned}
& \left\langle 1, n \| \mathbb{L}_{+} \mid 0, n\right\rangle=\langle 1, n \| i \sqrt{1(2 n+0)} \mid 1, n\rangle=i \sqrt{1(2 n+0)} \\
& \left\langle 2, n \| \mathbb{L}_{+} \mid 1, n\right\rangle=\langle 2, n \| i \sqrt{2(2 n+1)} \mid 2, n\rangle=i \sqrt{2(2 n+1)} \\
& \left\langle 3, n \| \mathbb{L}_{+} \mid 2, n\right\rangle=\langle 3, n \| i \sqrt{3(2 n+2)} \mid 3, n\rangle=i \sqrt{3(2 n+2)} \\
& \left\langle 4, n \| \mathbb{L}_{+} \mid 3, n\right\rangle=\langle 4, n \| i \sqrt{4(2 n+3)} \mid 4, n\rangle=i \sqrt{4(2 n+3)} \\
& \left\langle 5, n \| \mathbb{L}_{+} \mid 4, n\right\rangle=\langle 5, n \| i \sqrt{5(2 n+4)} \mid 5, n\rangle=i \sqrt{5(2 n+4)}
\end{aligned}
$$


For 5.j)

$$
\begin{aligned}
& \left\langle\mu, n \| \mathbb{L}_{-} \mid 0, n\right\rangle=0 \\
& \left\langle 0, n \| \mathbb{L}_{-} \mid 1, n\right\rangle=\left\langle\Phi_{0} \| i \sqrt{1(2 n+0)} \mid \Phi_{0}\right\rangle=i \sqrt{1(2 n+0)} \\
& \left\langle 1, n \| \mathbb{L}_{-} \mid 2, n\right\rangle=\left\langle\Phi_{1} \| i \sqrt{2(2 n+1)} \mid \Phi_{1}\right\rangle=i \sqrt{2(2 n+1)} \\
& \left\langle 2, n \| \mathbb{L}_{-} \mid 3, n\right\rangle=\left\langle\Phi_{2} \| i \sqrt{3(2 n+2)} \mid \Phi_{2}\right\rangle=i \sqrt{3(2 n+2)} \\
& \left\langle 3, n \| \mathbb{L}_{-} \mid 4, n\right\rangle=\left\langle\Phi_{3} \| i \sqrt{4(2 n+3)} \mid \Phi_{3}\right\rangle=i \sqrt{4(2 n+3)}
\end{aligned}
$$

By means of (4), (5), obtain

$$
\begin{aligned}
\left\langle\mu, n \| \mathbb{L}_{1} \mid m, n\right\rangle & =\frac{1}{2}\left\langle\mu, n \|\left(\mathbb{L}_{+}+\mathbb{L}_{-}\right) \mid m, n\right\rangle \\
& =\frac{1}{2}\left(i \sqrt{(m+1)(2 n+m)} \delta_{\mu, m+1}+i \sqrt{m(2 n+m-1)} \delta_{\mu, m-1}\right) \\
\left\langle\mu, n \| \mathbb{L}_{2} \mid m, n\right\rangle & =\frac{1}{2 i}\left\langle\mu, n \|\left(\mathbb{L}_{+}-\mathbb{L}_{-}\right) \mid m, n\right\rangle \\
& =\frac{1}{2}\left(\sqrt{(m+1)(2 n+m)} \delta_{\mu, m+1}-\sqrt{m(2 n+m-1)} \delta_{\mu, m-1}\right)
\end{aligned}
$$

From (4-86), obtain

$$
\left\langle m, n|| \mathbb{L}_{3} \mid m, n\right\rangle=(m+n)|m, n\rangle
$$

From (4-85), obtain

$$
\left\langle m, n \| \mathbb{L}^{2} \mid m, n\right\rangle=n(n-1)|m, n\rangle
$$

From (4-81),(4-82), obtain

then we have

$$
\begin{aligned}
& \left\langle m, n \| \mathbb{L}_{+} \mathbb{L}_{-} \mid m, n\right\rangle=-m(2 n+m-1) \\
& \left\langle m, n \| \mathbb{L}_{-} \mathbb{L}_{+} \mid m, n\right\rangle=-(m+1)(2 n+m)
\end{aligned}
$$

$$
\begin{aligned}
\left\langle m, n|| \mathbb{L}_{+} \mathbb{L}_{-}-\mathbb{L}_{-} \mathbb{L}_{+} \mid m, n\right\rangle & =2(m+n)=2\left\langle m, n \| \mathbb{L}_{3} \mid m, n\right\rangle \\
\left\langle m, n|| \mathbb{L}_{-} \mathbb{L}_{+}+\mathbb{L}_{-} \mathbb{L}_{+} \mid m, n\right\rangle & =\left\{n(n-1)-(m+n)^{2}\right\} \\
& =2\left(\left\langle m, n|| \mathbb{L}^{2} \mid m, n\right\rangle-\left\langle m, n \| \mathbb{L}_{3}^{2} \mid m, n\right\rangle\right) \\
& =2\left\langle m, n|| \mathbb{L}_{1}^{2}+\mathbb{L}_{2}^{2} \mid m, n\right\rangle
\end{aligned}
$$

5.2 Semi-Infinite Dimensional Matrix Element Representations $\Pi_{+}^{(-)}, \Pi_{-}^{(-)}, \Pi_{3}^{(-)}$, $\left(\Pi^{(-)}\right)^{2}$ of $\mathbb{L}_{+}, \mathbb{L}_{-}, \mathbb{L}_{3}, \mathbb{L}^{2}$, which arising from Spinor Ground State Family $\Delta_{m}^{-2 m_{0}}$

We will set up some tables which based on the matrix elements obtained in the previous work, then use these tables to make out semi-infinite dimension matrix $\Pi_{j}^{(-)}$. 
Using the series of matrix elements (4.j) (5.j), obtain the table1,table2 table1 $\left\langle\mu, n \| \mathbb{L}_{+} \mid m, n\right\rangle \Leftrightarrow$ matrix $\Pi_{+}^{(-)}$

\begin{tabular}{|ccccccc|}
\hline$\left\langle\mu, n \| \mathbb{L}_{+} \mid m, n\right\rangle$ & $|0, n\rangle$ & $|1, n\rangle$ & $|2, n\rangle$ & $|3, n\rangle$ & $|4, n\rangle$ \\
$\langle 0, n \|$ & 0 & 0 & 0 & 0 & 0 & $\cdot$ \\
$\langle 1, n \|$ & $i \sqrt{2 n}$ & 0 & 0 & 0 & 0 & $\cdot$ \\
$\langle 2, n \|$ & 0 & $i \sqrt{2(2 n+1)}$ & 0 & 0 & 0 & $\cdot$ \\
$\langle 3, n \|$ & 0 & 0 & $i \sqrt{3(2 n+2)}$ & 0 & 0 & $\cdot$ \\
$\langle 4, n \|$ & 0 & 0 & 0 & $i \sqrt{4(2 n+3)}$ & 0 &. \\
$\ldots$ & $\ldots$ & $\ldots$ & $\ldots$ & $\ldots$ & $\ldots$ \\
\hline
\end{tabular}

table2 $\left\langle\mu, n \| \mathbb{L}_{-} \mid m, n\right\rangle \Leftrightarrow$ matrix $\Pi_{-}^{(-)}$

\begin{tabular}{|ccccccc|}
\hline$\left\langle\mu, n \| \mathbb{L}_{-} \mid m, n\right\rangle$ & $|0, n\rangle$ & $|1, n\rangle$ & $|2, n\rangle$ & $|3, n\rangle$ & $|4, n\rangle$ &. \\
$\langle 0, n \|$ & 0 & $i \sqrt{2 n}$ & 0 & 0 & 0 &. \\
$\langle 1, n \|$ & 0 & 0 & $i \sqrt{2(2 n+1)}$ & 0 & 0 &. \\
$\langle 2, n \|$ & 0 & 0 & 0 & $i \sqrt{3(2 n+2)}$ & 0 &. \\
$\langle 3, n \|$ & 0 & 0 & 0 & 0 & $i \sqrt{4(2 n+3)}$ &. \\
$\langle 4, n \|$ & 0 & 0 & 0 & 0 & 0 &. \\
$\ldots$ & $\ldots$ & $\ldots$ & $\ldots$ & $\ldots$ & $\ldots$ &. \\
\hline
\end{tabular}

Using matrix elements (8), obtain

table $3\left\langle\mu, n \| \mathbb{L}_{3} \mid m, n\right\rangle \Leftrightarrow$ matrix $\Pi_{3}^{(-)}$

\begin{tabular}{|c|c|c|c|c|c|}
\hline$\left\langle\mu, n|| \mathbb{L}_{3} \mid m, n\right\rangle$ & $|0, n\rangle$ & $|1, n\rangle$ & $|2, n\rangle$ & $|3, n\rangle$ & $|4, n\rangle$ \\
\hline$\langle 0, n \|$ & $n$ & 0 & 0 & 0 & 0 \\
\hline$\langle 1, n \|$ & 0 & $n+1$ & 0 & 0 & 0 \\
\hline$\langle 2, n \|$ & 0 & 0 & $n+2$ & 0 & 0 \\
\hline$\langle 3, n \|$ & 0 & 0 & 0 & $n+3$ & 0 \\
\hline$\langle 4, n \|$ & 0 & 0 & 0 & 0 & $n+4$ \\
\hline$\bullet \cdot$ & $\bullet \cdot$ & $\cdots$ & $\cdots \cdot$ & $\cdots$ & $\cdots$ \\
\hline
\end{tabular}

Using matrix elements (9), obtain

table $4\left\langle\mu, n \| \mathbb{L}^{2} \mid m, n\right\rangle \Leftrightarrow$ matrix $\left(\Pi^{(-)}\right)^{2}$

\begin{tabular}{|ccccccc|}
\hline$\left\langle\mu, n \| \mathbb{L}^{2} \mid m, n\right\rangle$ & $|0, n\rangle$ & $|1, n\rangle$ & $|2, n\rangle$ & $|3, n\rangle$ & $|4, n\rangle$ & $\cdot$ \\
$\langle 0, n \|$ & $n(n-1)$ & 0 & 0 & 0 & 0 & $\bullet$ \\
$\langle 1, n \|$ & 0 & $n(n-1)$ & 0 & 0 & 0 & $\bullet$ \\
$\langle 2, n \|$ & 0 & 0 & $n(n-1)$ & 0 & 0 & $\bullet$ \\
$\langle 3, n \|$ & 0 & 0 & 0 & $n(n-1)$ & 0 & $\bullet$ \\
$\langle 4, n \|$ & 0 & 0 & 0 & 0 & $n(n-1)$ & $\bullet$ \\
$\cdots$ & $\cdots$ & $\cdots$ & $\cdots$ & $\cdots$ & $\cdots$ & $\bullet$ \\
\hline
\end{tabular}


5.3 Semi-Infinite Dimensional Matrix Element Representations $\Pi_{+}^{(+)}, \Pi_{-}^{(+)}, \Pi_{3}^{(+)}$, $\left(\Pi^{(+)}\right)^{2}$ of $\mathbb{L}_{+}, \mathbb{L}_{-}, \mathbb{L}_{3}, \mathbb{L}_{+}^{2}$ which arising from Spinor Ground State Family $\Delta_{m}^{+2 m_{0}}$

On the analogy of the above table1,2,3,4 related to $\Pi_{j}^{(-)}$, which arising from $\Delta_{m}^{-2 m_{0}}$, table5,6,7,8 related to matrix $\Pi_{j}^{(+)}$, which from $\Delta_{m}^{+2 m_{0}}$, could be obtained:

table5 $\left\langle-\mu, n \| \mathbb{L}_{+} \mid-m, n\right\rangle \Leftrightarrow$ matrix $\Pi_{+}^{(+)}$

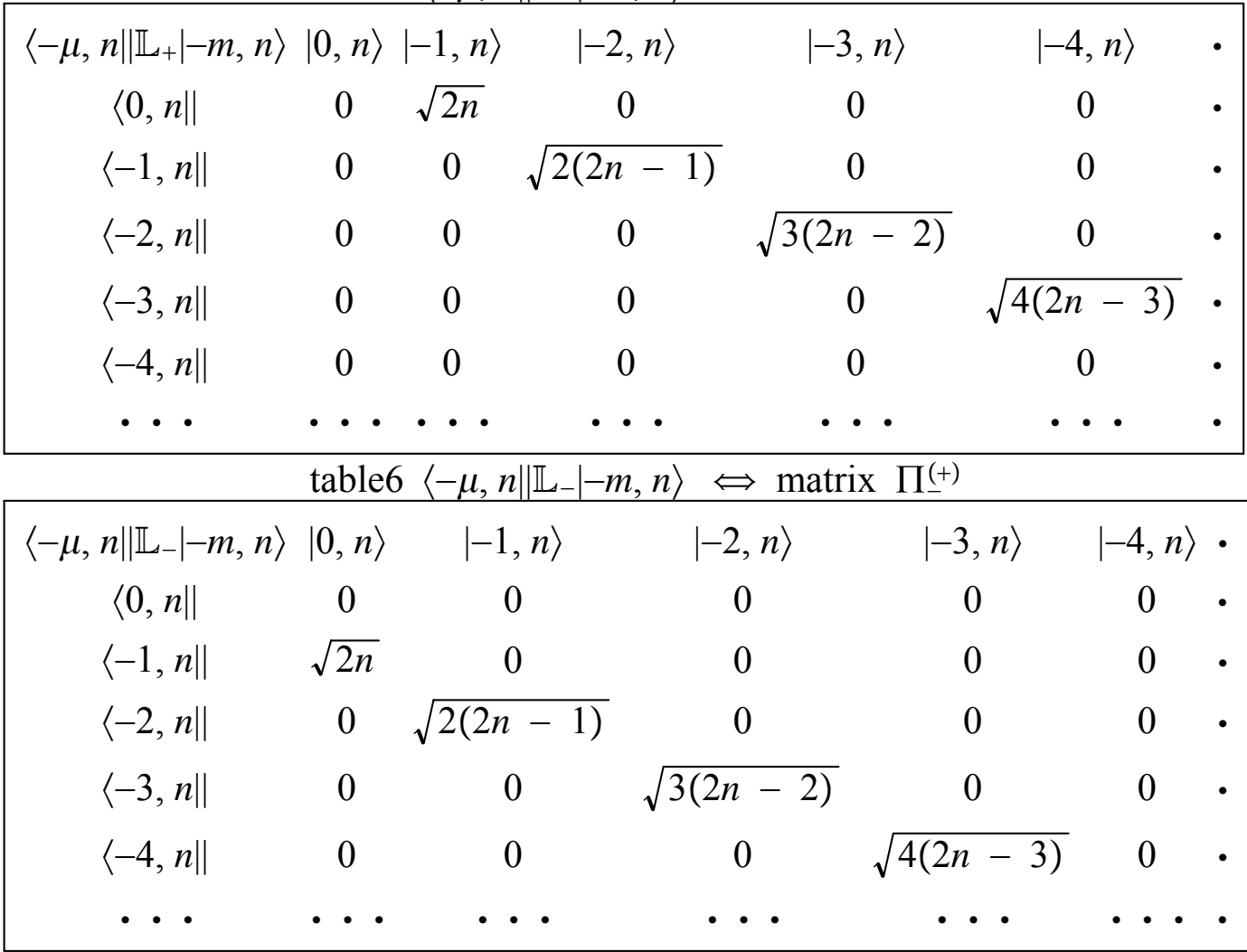

table7 $\left\langle-\mu, n \| \mathbb{L}_{3} \mid-m, n\right\rangle \Leftrightarrow$ matrix $\Pi_{3}^{(+)}$

$\left\langle-\mu, n\left|\mathbb{L}_{3}\right|-m, n\right\rangle|0, n\rangle|-1, n\rangle|-2, n\rangle|-3, n\rangle|-4, n\rangle$.

$\langle 0, n \| \quad n \quad 0 \quad 0 \quad 0 \quad 0 \quad 0$

$\langle-1, n \| \quad 0 \quad n-1 \quad 0 \quad 0 \quad 0 \quad 0$

$\langle-2, n \| \quad 0 \quad 0 \quad n-2 \quad 0 \quad 0$

$\langle-3, n \| \quad 0 \quad 0 \quad 0 \quad n-3 \quad 0$

$\langle-4, n \| \quad 0 \quad 0 \quad 0 \quad 0 \quad n-4 \cdot$

... .............

table8 $\left\langle-\mu, n \| \mathbb{L}^{2} \mid-m, n\right\rangle \Leftrightarrow$ matrix $\left(\Pi^{(+)}\right)^{2}$

\begin{tabular}{ccccccc|}
$\left\langle-\mu, n \| \mathbb{L}^{2} \mid-m, n\right\rangle$ & $|0, n\rangle$ & $|-1, n\rangle$ & $|-2, n\rangle$ & $|-3, n\rangle$ & $|-4, n\rangle$ & $\cdot$ \\
$\langle 0, n \|$ & $n(n+1)$ & 0 & 0 & 0 & 0 & $\cdot$ \\
$\langle-1, n \|$ & 0 & $n(n+1)$ & 0 & 0 & 0 &. \\
$\langle-2, n \|$ & 0 & 0 & $n(n+1)$ & 0 & 0 & $\cdot$ \\
$\langle-3, n \|$ & 0 & 0 & 0 & $n(n+1)$ & 0 &. \\
$\langle-4, n \|$ & 0 & 0 & 0 & 0 & $n(n+1)$ & $\cdot$ \\
$\ldots$ & $\ldots$ & $\ldots$ & $\ldots$ & $\ldots$ & $\ldots$ &.
\end{tabular}


5.4 Matrices $\vec{\Pi}_{j}^{(+)}, \vec{\Pi}_{j}^{(-)}$

Right-circumrotatory spin matrix $\vec{\Pi}_{j}^{(+)}$comes from the same way of $\vec{\Pi}_{j}^{(-)}$

$$
\begin{aligned}
& \mathbb{L}_{+}|-m, n\rangle=\sqrt{m(2 n-m+1)}|-m+1, n\rangle \\
& \mathbb{L}_{-}|-m, n\rangle=\sqrt{(m+1)(2 n-m)}|-m-1, n\rangle
\end{aligned}
$$

Where

$$
\begin{aligned}
2 n & =2 n_{+}=-t=+4 m_{0} \\
m & =0,1,2,3, \ldots \ldots
\end{aligned}
$$

Left-circumrotatory spin matrix $\vec{\Pi}_{j}^{(-)}$results in (4-76), (4-77)

$$
\begin{aligned}
& \mathbb{L}_{+}|+m, n\rangle=i \sqrt{(m+1)(2 n+m)}|+m+1, n\rangle \\
& \mathbb{L}_{-}|+m, n\rangle=i \sqrt{m(2 n+m-1)}|+m+1, n\rangle
\end{aligned}
$$

Where

$$
\begin{aligned}
2 n & =2 n_{-}=-s=-4 m_{0} \\
m & =0,1,2,3, \ldots \ldots
\end{aligned}
$$

$\vec{\Pi}_{j}^{(+)}$and $\vec{\Pi}_{j}^{(-)}$are angular momentum operators, which satisfy angular momentum commutation relations.

$$
\vec{\Pi}_{j}^{( \pm)} \times \vec{\Pi}_{j}^{( \pm)}=i \vec{\Pi}_{j}^{( \pm)} \quad j=1,2,3
$$




\title{
6 Infinite Dimensional Representations: $\xi_{j, n}^{(\oplus)}$ Chaos Spin Hierarchy (CSH)
}

\author{
Infinite Dimensional Representations $\xi_{j, n}^{( \pm)}$are called Chaos Spin Hierarchy \\ (CSH)
}

6.1 Recalling spin hierarchy (SH), $\Pi^{(+)}$and $\Pi^{(-)}$(in previous chapter), that arise from the matrix elements table5, 6, 7, 8 and table1, 2, 3, 4 of orbital angular momentum operators $\mathbb{L}_{+}, \mathbb{L}_{-}, \mathbb{L}_{3}, \mathbb{L}^{2}$.

These matrix elements marked by indexes $\mu$ and $m$, which appear in the $\mu$ th row and the $m$ th column, are shown in table9. Where quantum numbers $\mu, m \geqslant 0$, $\mu$ and $m$ vary from zero to positive infinite. The minimum of $\mu=m$ is zero, which lies at the most top left corner $\circ$ of all matrix elements.

Table9 Spin Hierarchy down-semi-infinite dimensional matrix elements

\begin{tabular}{|c|c|c|c|c|c|c|}
\hline & & $\begin{array}{c}\Pi_{j, n}^{( \pm)} \\
\left\langle\mp \mu, n|| \mathbb{L}_{+}, \mathbb{L}_{-}, \mathbb{L}_{3}, \mathbb{L}^{2} \mid \mp m, n\right\rangle\end{array}$ & $\begin{array}{c}m=0 \\
|0, n\rangle\end{array}$ & $\begin{array}{l}m=1 \\
|\mp 1, n\rangle\end{array}$ & $\begin{array}{l}m=2 \\
|\mp 2, n\rangle\end{array}$ & • \\
\hline$\mu$ & $=0$ & $\langle 0, n \|$ & 00 & $\circ \circ$ & $\circ \circ$ & • \\
\hline$\mu$ & $=1$ & $\langle\mp 1, n \|$ & $\circ \circ$ & $\circ \circ$ & $\circ \circ$ & • \\
\hline$\mu$ & $=2$ & $\langle\mp 2, n \|$ & $\circ \circ$ & $\circ \circ$ & $\circ \circ$ & • \\
\hline & • & • & • & • & • & • \\
\hline & • & • & • & • & • & \\
\hline
\end{tabular}

If remove the restrictions on the values of $\mu$ and $m$ in table9, and postulate that $\mu m$ could be greater or less than zero, then down-semi-infinite dimensional matrices $\Pi_{j, n}^{( \pm)}$will turn to infinite dimensioal matrices $\xi_{j, n}^{( \pm)} \equiv \xi_{j, n_{ \pm}}$. then table9 turns to table10 and table11

table10 Spin Hierarchy from $\mathrm{SH}$ to $\mathrm{CSH}$

\begin{tabular}{|ccc|}
\hline Spin Hierarchy $(\mathrm{SH}) \Pi_{j, n}^{( \pm)}$ & $\Rightarrow$ & Chaos Spin Hierarchy $(\mathrm{CSH}) \xi_{j, n}^{( \pm)}$ \\
down-semi-infinite dimensional matrix & $\Rightarrow$ & infinite dimensioal matrix \\
$\mu, m=0,1,2,3, \ldots \ldots+\infty \geqslant 0$ & $\Rightarrow$ & $\mu, m=0, \pm 1, \pm 2, \pm 3, \ldots \ldots \pm \infty$ \\
\hline
\end{tabular}


Table11 Hierarchy infinite dimensional matrix elements of Chaos Spin

\begin{tabular}{|c|c|c|c|c|c|c|c|c|c|c|c|}
\hline & & $\begin{array}{c}\xi_{j, n}^{( \pm)} \\
\left\langle\mp \mu, n|| \mathbb{L}_{+}, \mathbb{L}_{-}, \mathbb{L}_{3}, \mathbb{L}^{2} \mid \mp m, n\right\rangle\end{array}$ & . & $\begin{array}{l}m= \\
\mid \pm 2\end{array}$ & $\begin{array}{l}=-2 \\
, n\rangle\end{array}$ & $\begin{array}{l}m= \\
\pm 1,\end{array}$ & $n>$ & $\begin{array}{l}m=0 \\
|0, n\rangle\end{array}$ & $\begin{array}{l}n=+ \\
|\mp 1, n\rangle\end{array}$ & $\begin{array}{l}m= \\
\mid \mp 2,\end{array}$ & $\begin{array}{l}+2 \\
n\rangle\end{array}$ \\
\hline & • & • & • & & • & • & & • & • & - & \\
\hline & • & • & • & & • & • & & • & • & - & \\
\hline$\mu$ & $=-2$ & $\langle \pm 2, n \|$ & • & $\times$ & $x$ & $\times$ & $\times$ & $\times \times$ & $\times \times$ & $\times$ & \\
\hline$\mu$ & $=-1$ & $\langle \pm 1, n \|$ & • & $\times$ & $\times$ & $\times$ & $\times$ & $\times \times$ & $\times \times$ & $\times$ & \\
\hline$\mu$ & $=0$ & $\langle 0, n \|$ & - & $\times$ & $\times$ & $\times$ & $\times$ & 00 & $\circ \circ$ & $\circ$ & $\circ$ \\
\hline$\mu$ & $=+1$ & $\langle\mp 1, n \|$ & • & $\times$ & $x$ & $\times$ & $\times$ & $\circ \circ$ & $\circ \circ$ & $\circ$ & $\circ$ \\
\hline$\mu$ & $=+2$ & $\langle\mp 2, n \|$ & • & $\times$ & $\times$ & $x$ & $\times$ & $\circ \circ$ & $\circ 0$ & $\circ$ & $\circ$ \\
\hline & • & • & • & & • & • & & • & • & - & 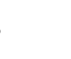 \\
\hline & • & - & • & & • & • & & • & • & • & \\
\hline
\end{tabular}

Call attentation to the following pair of correpondences:

\begin{tabular}{|c|}
\hline Spin Hierarchy \\
matrices $\Pi_{j, n}^{( \pm)}$ \\
$\mu, m$ \\
$=0, \ldots+\infty$ \\
in table 9
\end{tabular}

\section{Chaos Spin Hierarchy} matrices $\xi_{j, n}^{( \pm)}$ $\mu, m$
$=-\infty, \ldots, 0, \ldots+\infty$ in table 11

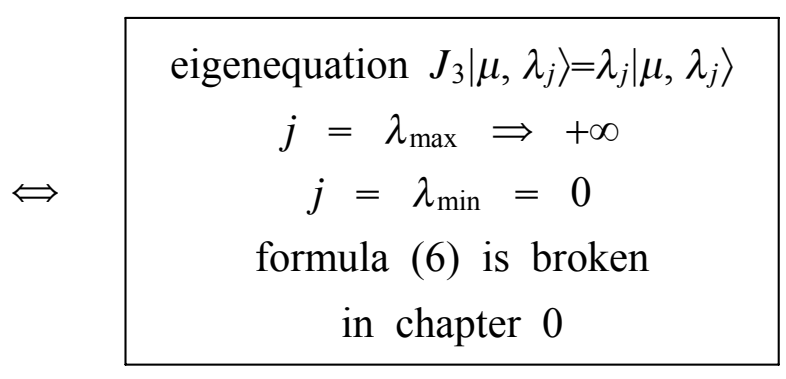

$\Leftrightarrow \quad \begin{gathered}\text { eigenequation } J_{3}\left|\mu, \lambda_{j}\right\rangle=\lambda_{j}\left|\mu, \lambda_{j}\right\rangle \\ j=\lambda_{\max } \Rightarrow+\infty \\ j=\lambda_{\min } \Rightarrow-\infty \\ \text { formula (6) is broken } \\ \text { in chapter } 0\end{gathered}$

6.2 Extent of Spin Hierarchy matrices $\Pi_{j}^{( \pm)}$

\section{to Chaos Spin Hierarchy matrices $\xi_{j, n}^{( \pm)}$}

Using formulas $\mathbb{L}_{1}=\frac{1}{2}\left\{\mathbb{L}_{+}+\mathbb{L}_{-}\right\}$and $\mathbb{L}_{2}=\frac{1}{2 \mathrm{i}}\left\{\mathbb{L}_{+}-\mathbb{L}_{-}\right\}$:

1) From matrix elements of table5, table6, table 7, table 8 of $\mathbf{S H} \Pi_{j}^{(+)}$, we have Chaos Spin Hierarchy matrices $\xi_{j, n}^{(+)}$

2) From matrix elements of table1, table 2 , table 3 , table 4 of $\mathbf{S H} \Pi_{j}^{(-)}$, we have matrix representations of CSH $\xi_{j, n}^{(-)}$ 
1) For Chaos Spin Hierarchy $(\mathbf{C S H}) \xi_{j, n}^{(+)}\left(\operatorname{simplified} \xi_{j}\left(n_{+}=n\right)\right)$ $\xi_{1}\left(n_{+}=n\right)=\frac{1}{2}$

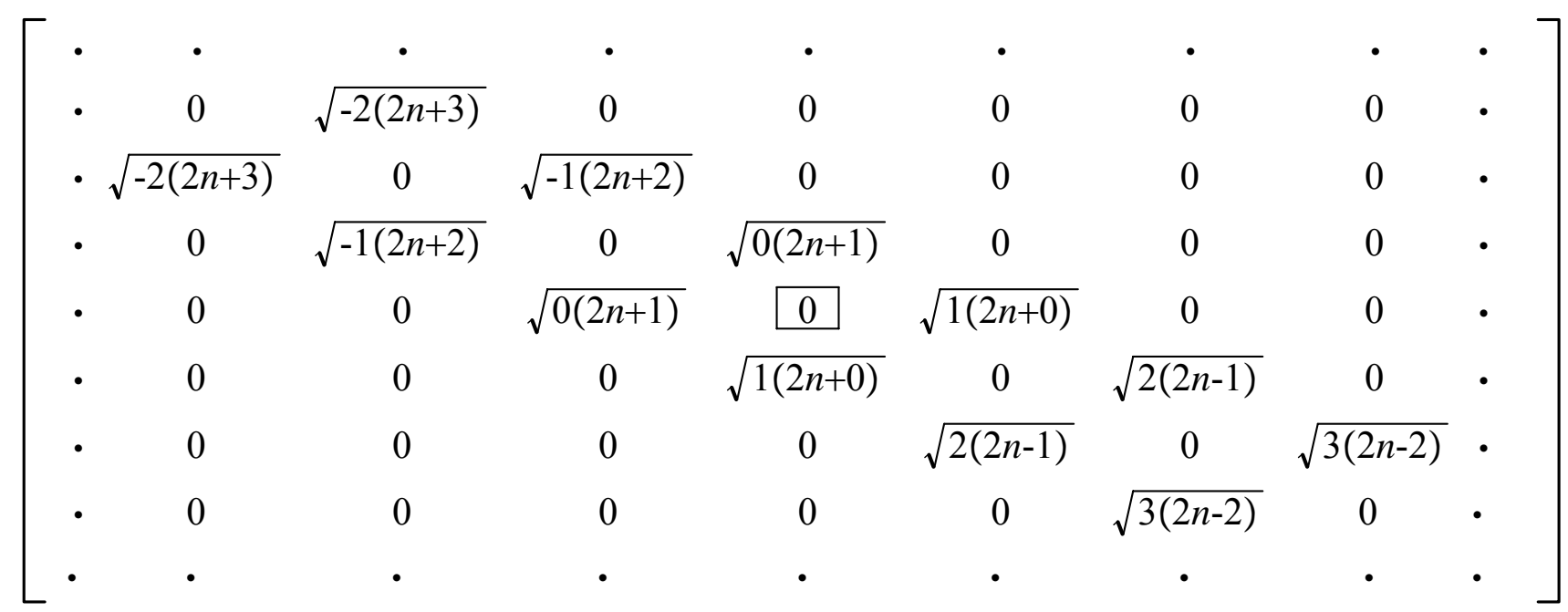

(1)

$\xi_{2}\left(n_{+}=n\right)=\frac{1}{2}$

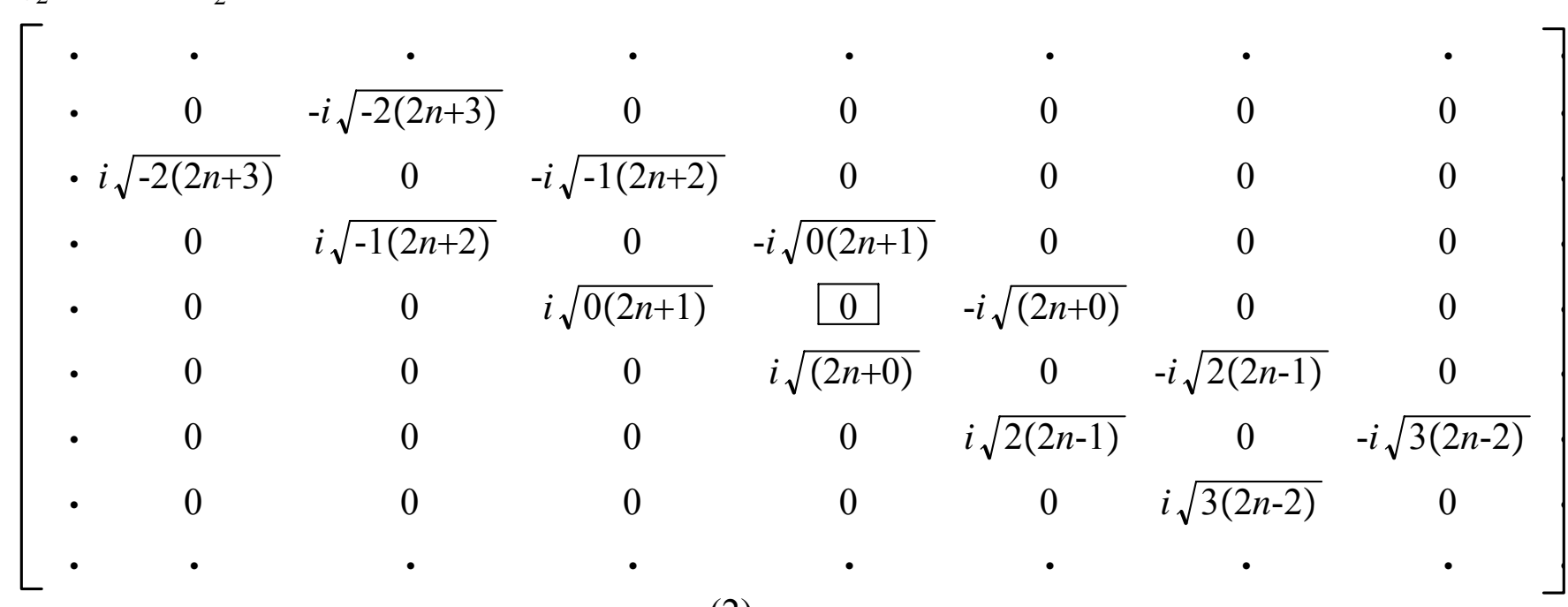

(2)

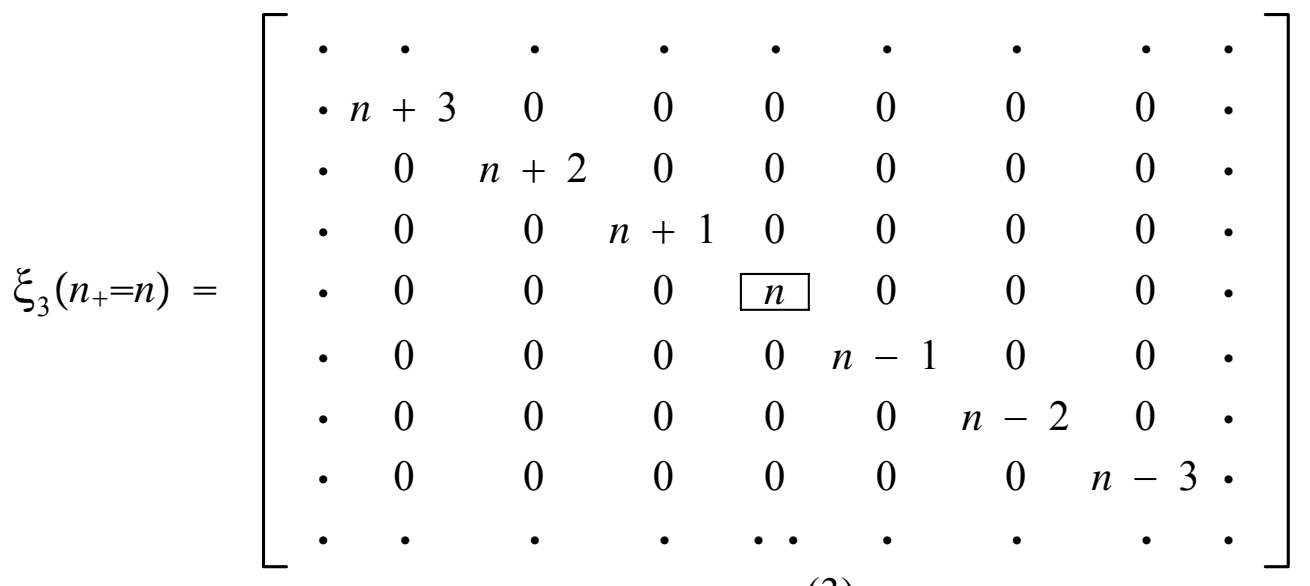

(3) 


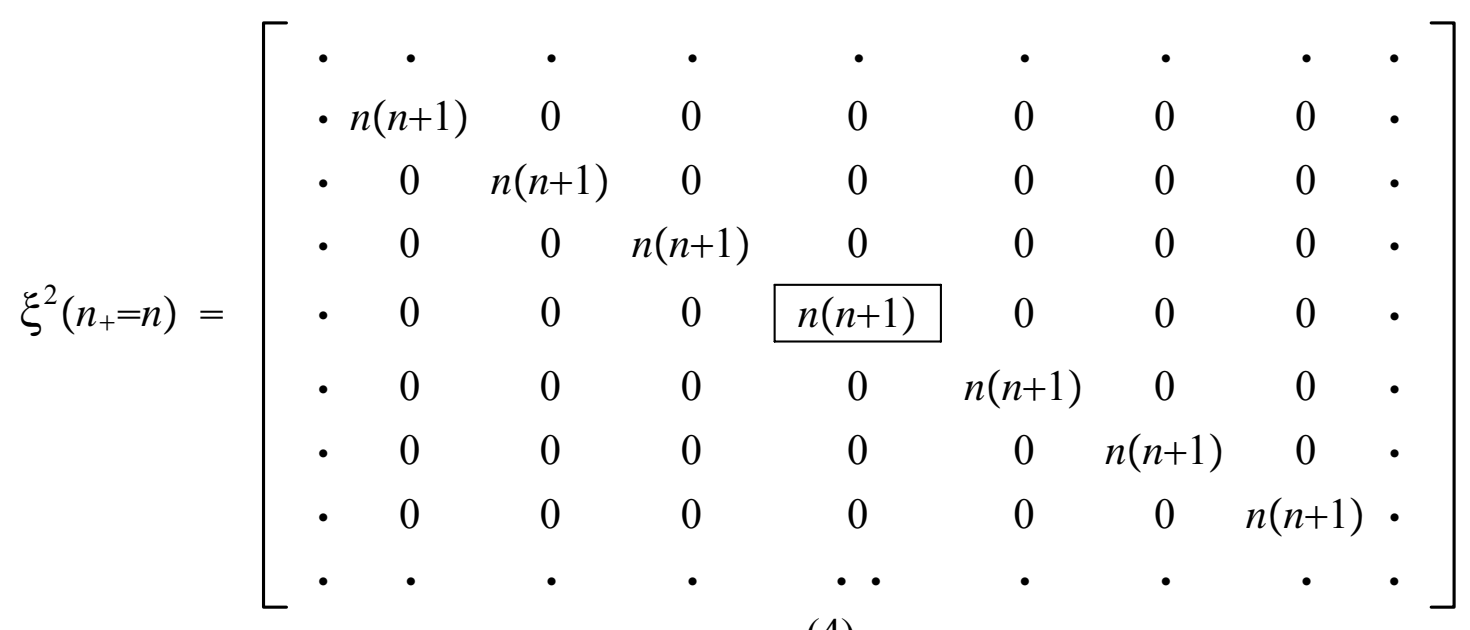

(4) 
2) For Chaos Spin Hierarchy $(\mathbf{C S H}) \xi_{j, n}^{(-)}\left(\operatorname{simplified} \xi_{j}\left(n_{-}=n\right)\right)$ $\xi_{1}\left(n_{-}=n\right)=\frac{1}{2}$

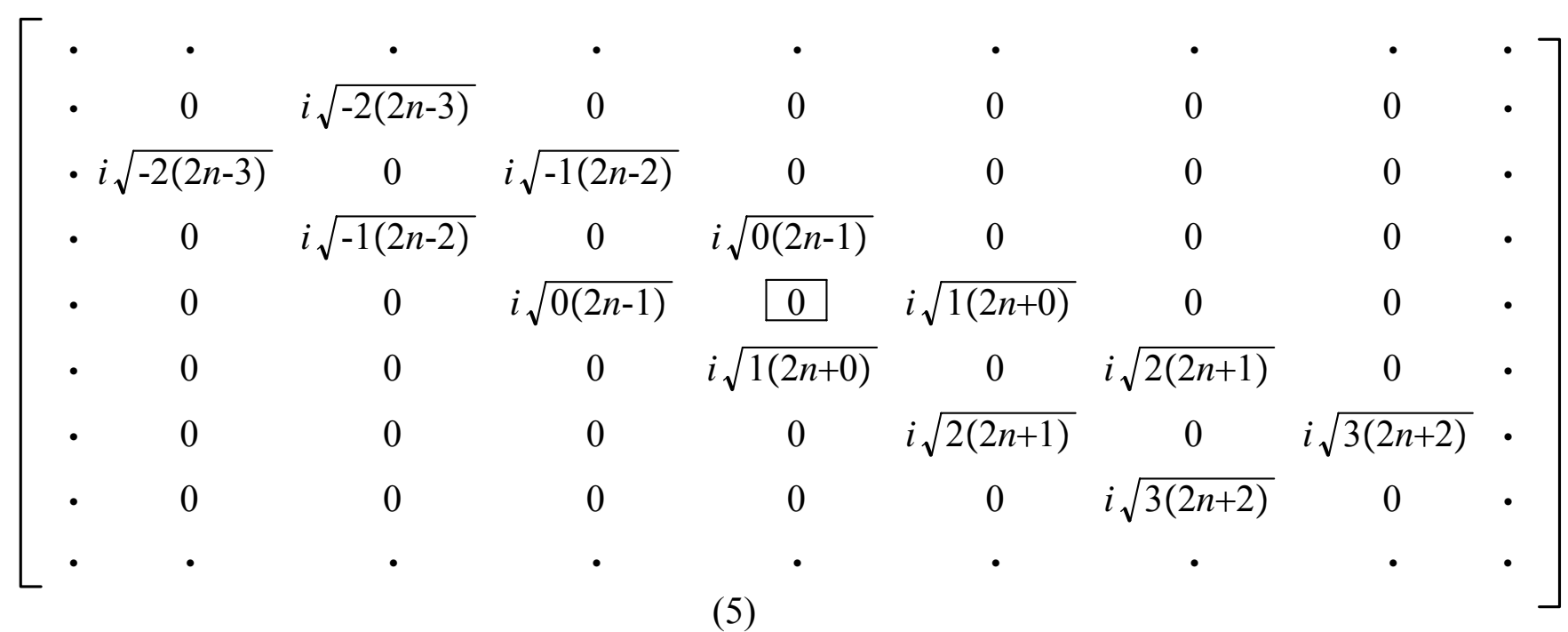

$\xi_{2}\left(n_{-}=n\right)=\frac{1}{2}$

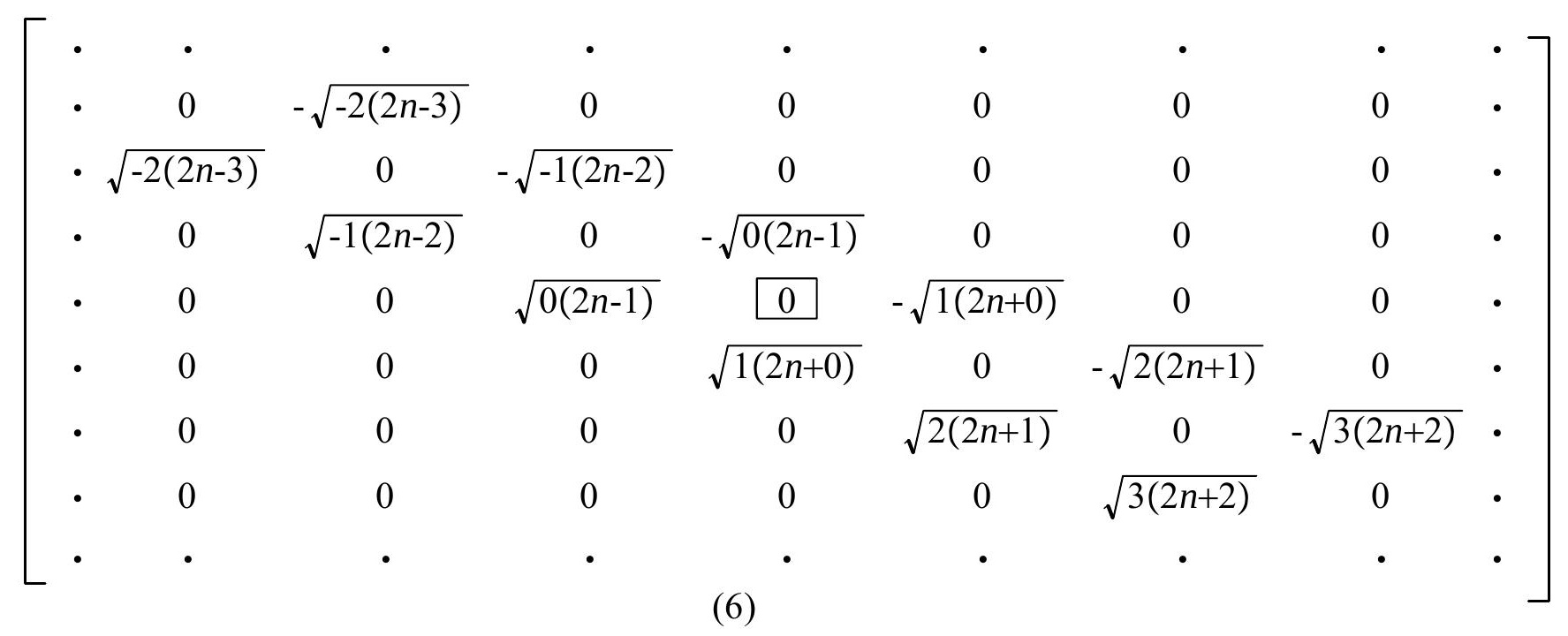

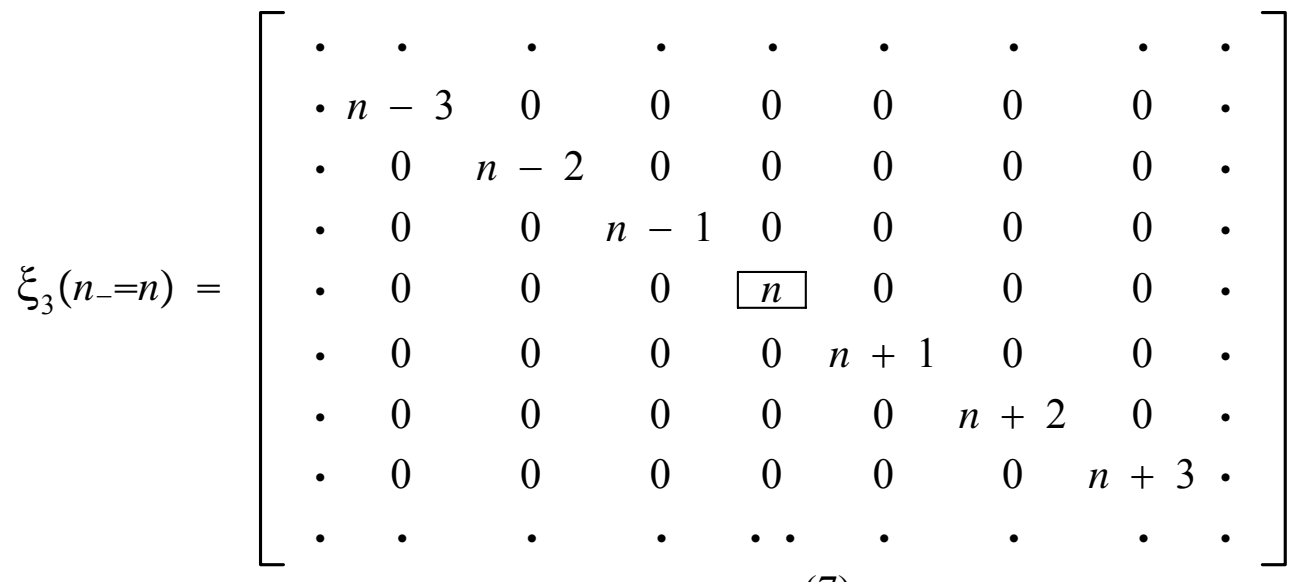




$$
\xi^{2}\left(n_{-}=n\right)=\left[\begin{array}{cccccccccc}
\bullet & \bullet & \bullet & \bullet & \bullet & \bullet & \bullet & \bullet & \bullet \\
\bullet & n(n-1) & 0 & 0 & 0 & 0 & 0 & 0 & \bullet \\
\bullet & 0 & n(n-1) & 0 & 0 & 0 & 0 & 0 & \bullet \\
\bullet & 0 & 0 & n(n-1) & 0 & 0 & 0 & 0 & \bullet \\
\bullet & 0 & 0 & 0 & n(n-1) & 0 & 0 & 0 & \bullet \\
\bullet & 0 & 0 & 0 & 0 & n(n-1) & 0 & 0 & \bullet \\
\bullet & 0 & 0 & 0 & 0 & 0 & n(n-1) & 0 & \bullet \\
\bullet & 0 & 0 & 0 & 0 & 0 & 0 & n(n-1) & \bullet \\
\bullet & \bullet & \bullet & \bullet & \bullet & \bullet & \bullet & \bullet & \bullet
\end{array}\right]
$$

There are two branches of Chaos Spin Hierarchy:

1) matrices $\xi_{1}\left(n_{+}\right)(1), \xi_{2}\left(n_{+}\right)(2), \xi_{3}\left(n_{+}\right)(3), \xi^{2}\left(n_{+}\right)(4)$ of $\xi_{j, n}^{(+)}$

2) matrices $\xi_{1}\left(n_{-}\right)(5), \xi_{2}\left(n_{-}\right)(6), \xi_{3}\left(n_{-}\right)(7), \xi^{2}\left(n_{-}\right)(8)$ of $\xi_{j, n}^{(-)}$

$\xi_{j}\left(n_{ \pm}\right)$are the fundamental roles in describing TKP's behavious we seek the objective of this paper is attained

Next paragraphs, we will give some explicit matrix representations of Chaos Spin Hierarchy, through three examples of $\xi_{j}\left(n_{+}=n\right)$ with $n_{+}=+0,+1 / 2,+1 / 3$. 


\section{Spin $0 \hbar \quad C S H\{0,+0,(+)\}, \Omega_{j,+0}$}

$$
\begin{array}{lrl}
\text { Symbol } & \{0,+0,(+)\} & \equiv\left\{\xi^{2}=0, n=+0, \quad(+)=n_{+}=n\right\} \\
\text { Here } & \Omega_{j,+0} \equiv \Omega_{j, n=n_{+}++0}^{(+)=n_{+}} \equiv \xi_{j,+0}^{(+)}
\end{array}
$$

7.1 Spin $0 \hbar$ particle is the simpliest rotational particle. In conventional quantum mechanics frame, the spin angular momentum operator of spin $0 \hbar$ particle is a zero-value $1 \times 1$ dimensional matrix. Its three components are as following

$$
S_{1,0}=S_{2,0}=S_{3,0}=0 \hbar
$$

The commutation rule is given below

$$
S_{j, 0} S_{k, 0}-S_{k, 0} S_{j, 0}=i \hbar S_{l, 0}
$$

Or:

$$
0_{j, 0} \cdot 0_{k, 0}-0_{k, 0} \cdot 0_{j, 0}=i \cdot 0_{l, 0}
$$

$\overrightarrow{0}=(0,0,0)$ is an indefinite orientational vector, but zero-value. $S_{j, 0}$ is a point model, lacks of stereo!

We will see that $S_{j, 0}$ actually just is the intrinsic angular momemtun of spin $0 \hbar$ particle, is merely the part of Island Ooperator $\Omega_{j,+0}=\xi_{j,+0}(15)$.

7.2 The following are the concrete expressions of $\Omega_{j,+0}$ base on (6-1),(6-2),(6-3)

Island operator $\Omega_{1,+0}=$

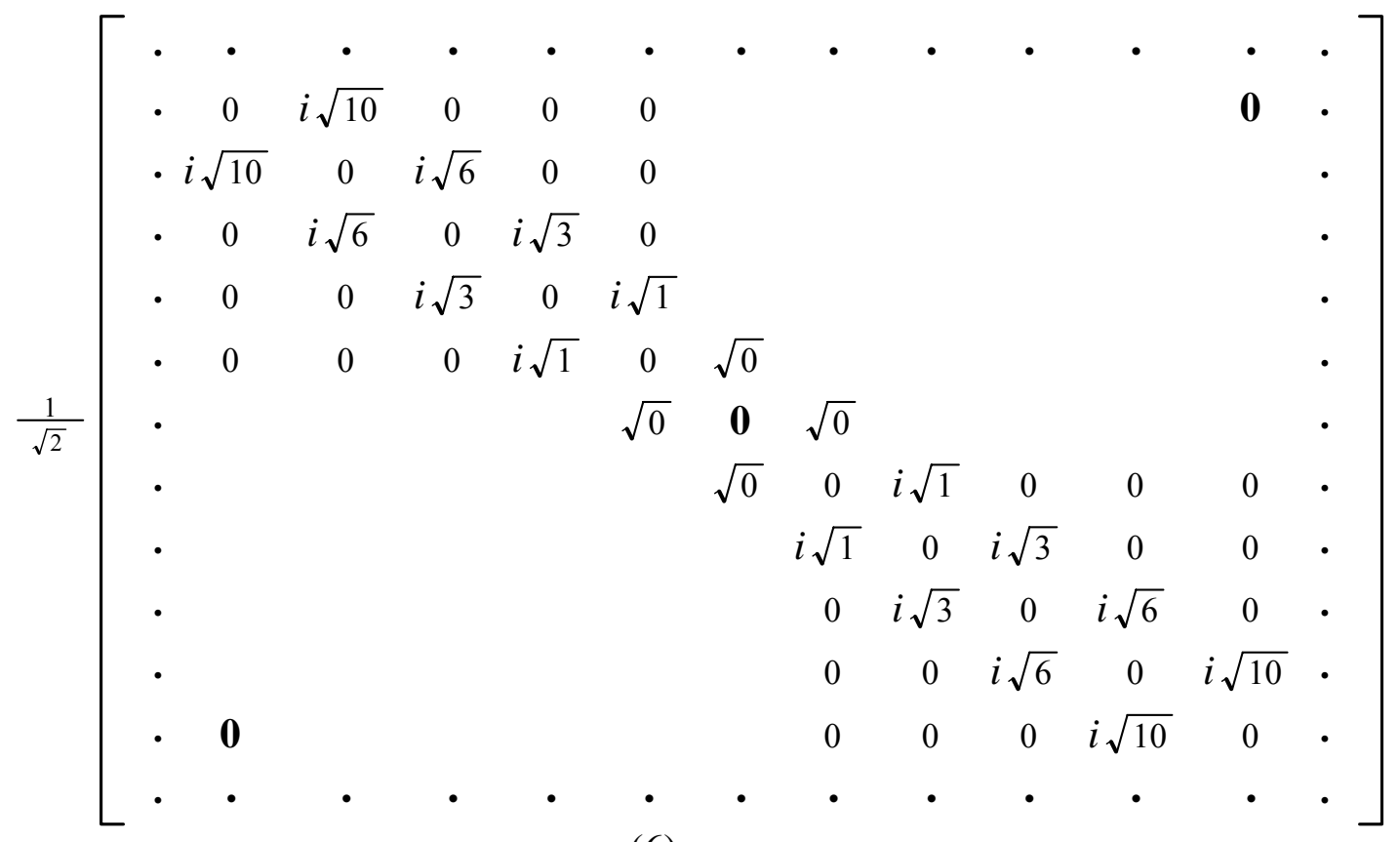


Island operator $\Omega_{2,+0}=$

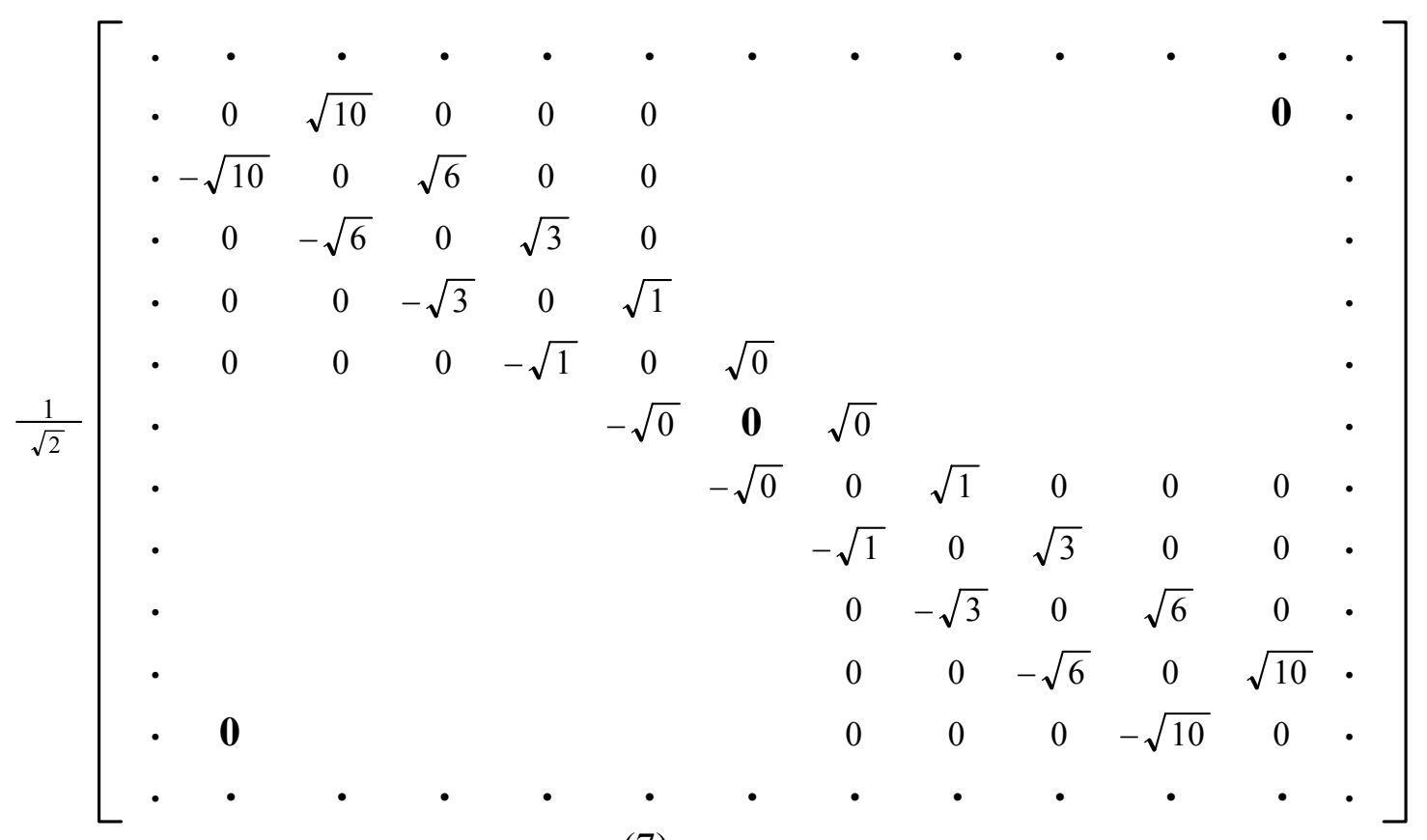

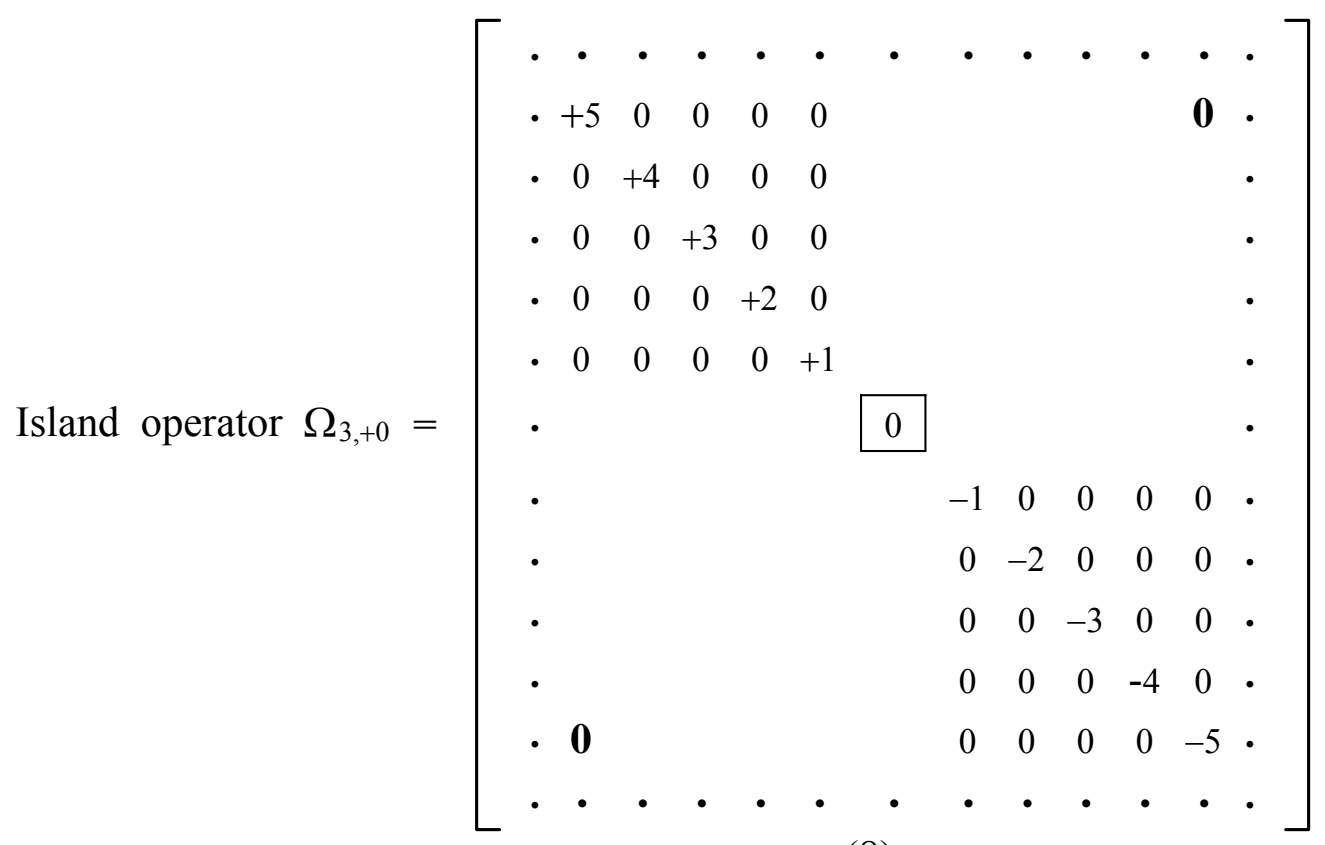

(8)

(6),(7),(8) obey angular momentum commutation relation

$$
\Omega_{j,+0} \Omega_{k,+0}-\Omega_{k,+0} \Omega_{j,+0}=i \Omega_{l,+0}, j, k . l=1,2,3
$$


7.3 Evaluation of $\Omega_{1,+0}^{2}, \Omega_{2,+0}^{2}, \Omega_{3+0}^{2}$ and $\Omega_{+0}^{2}$ get: $\Omega_{1,+0}^{2}=$

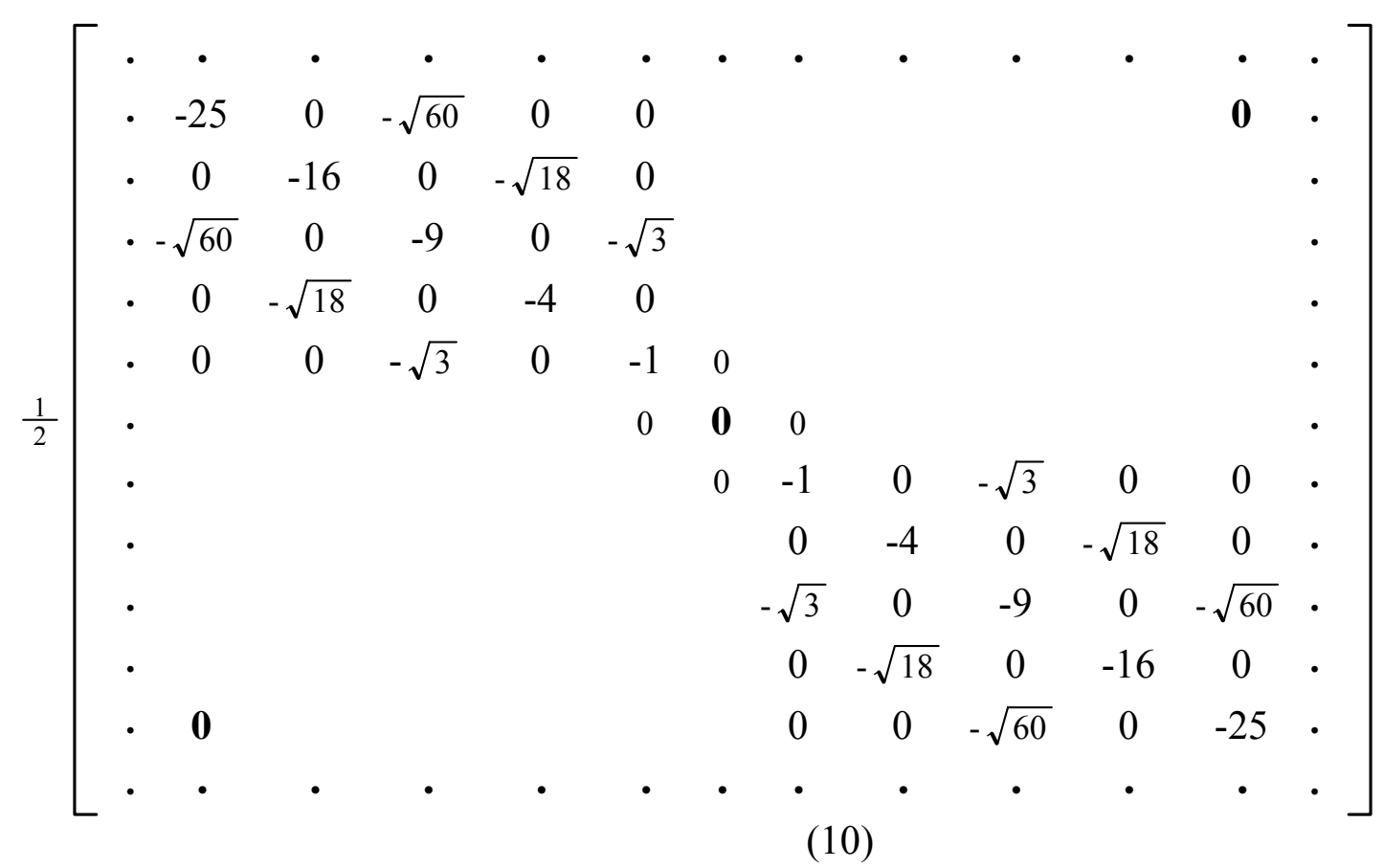

and $\Omega_{2,+0}^{2}=$

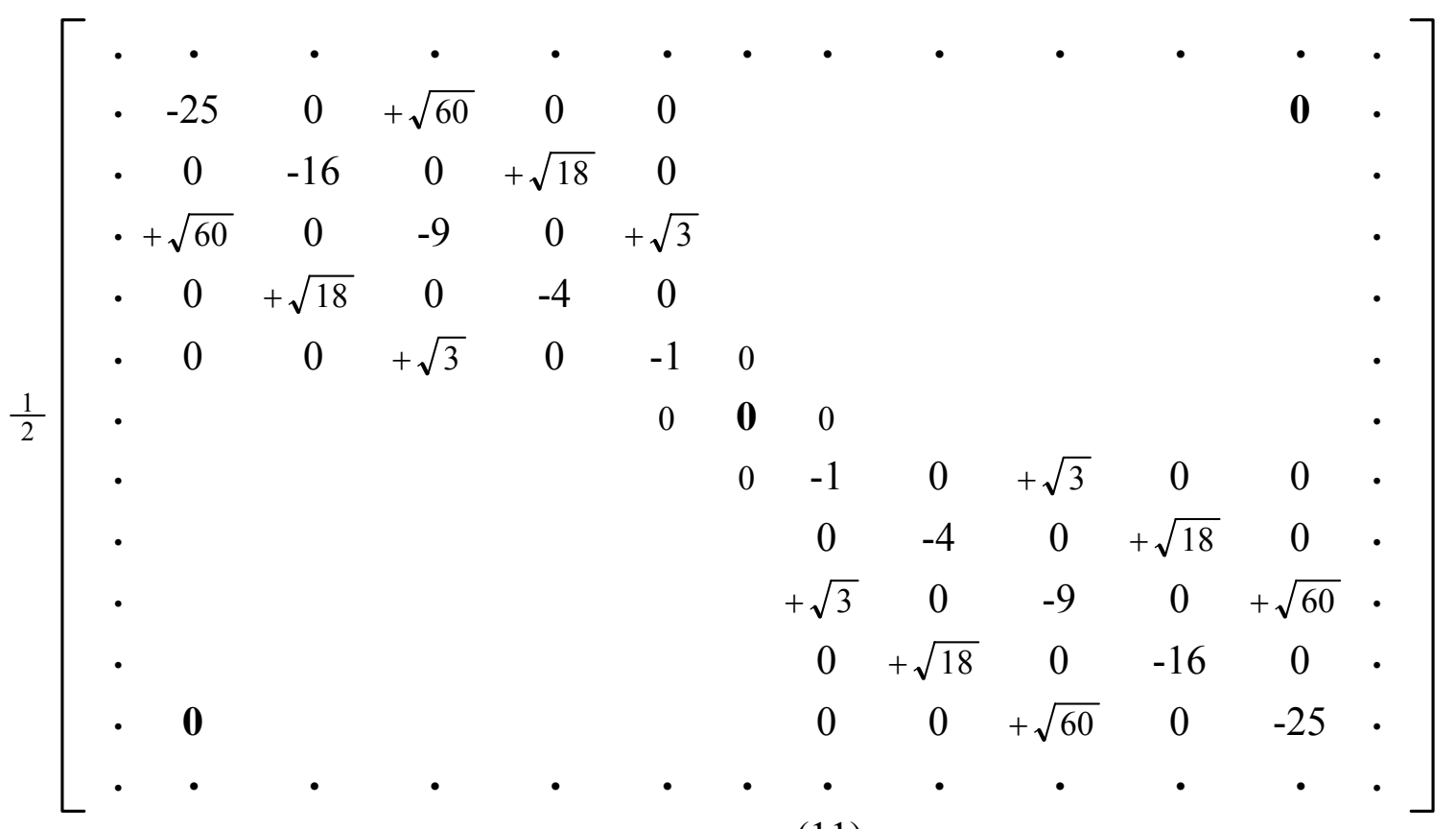


From (10) and (11), hence

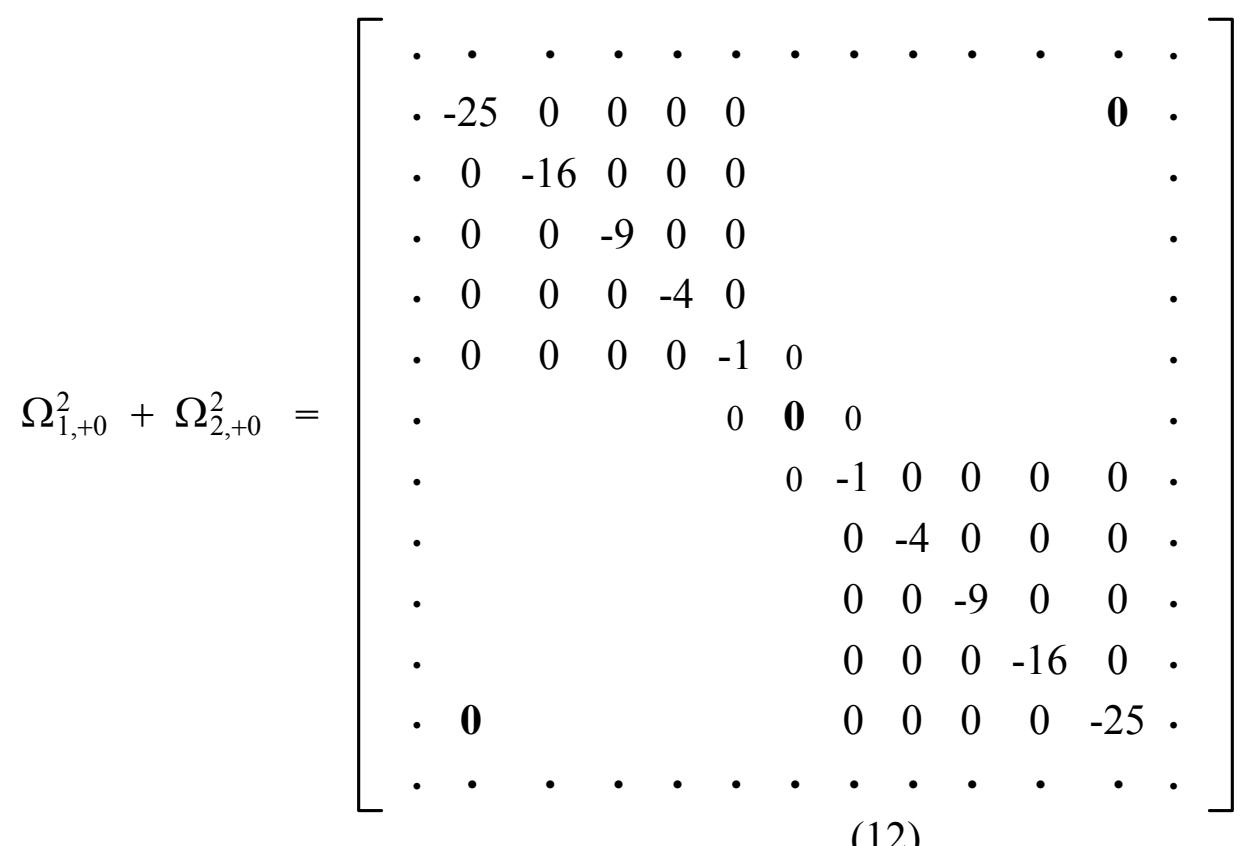

From (8), get

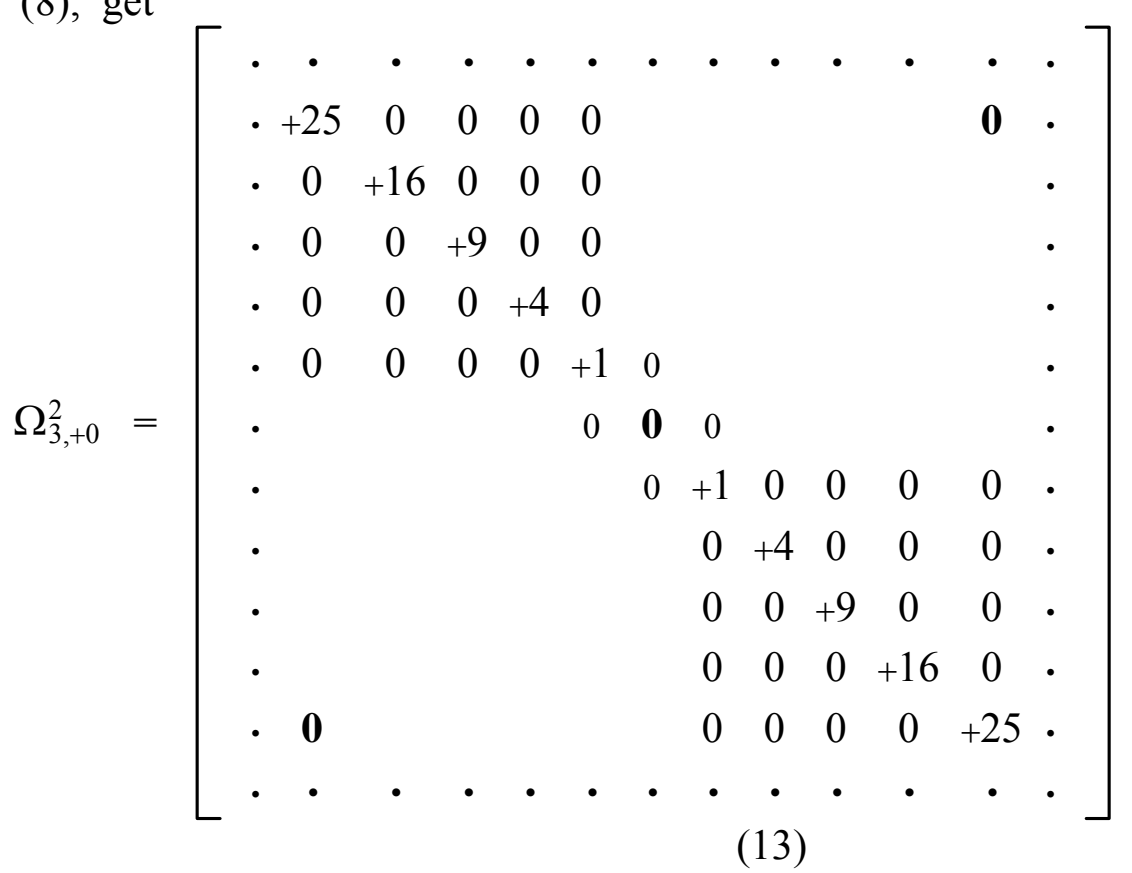


Although the eigenvalues of $\Omega_{1,+0}^{2}+\Omega_{2,+0}^{2}$ (12) are equal and less than zero, but the eigenvalues of $\Omega_{3}^{2}$ (13) are equal and greater than zero. Further the total square operator $\Omega_{+0}^{2}$ (14) remains to be a zero matrix

$$
\Omega_{+0}^{2}=\Omega_{1,+0}^{2}+\Omega_{1,+0}^{2}+\Omega_{1,+0}^{2}=0(0+1) I_{0}=0 \hbar^{2} I_{0}
$$

where $I_{0}$ ia an infinie dimensional uint matrix.

7.4 Island operators (6), (7), (8) can be written as $\Omega_{j,+0}$ as below. $(j=1,2,3)$

$$
\Omega_{j,+0}=\xi_{j,+0}=\left[\begin{array}{ccccc}
\Gamma_{j,+0}^{\mathbf{U}} \neq 0 & 0 & 0 & \\
0 & S_{j,+0}=S_{j, 0}=0 & 0 & \\
0 & & 0 & \Gamma_{j,+0}^{\mathbf{D}} \neq 0
\end{array}\right]
$$

$\Gamma_{j,+0}^{\mathbf{U}}: \quad$ Up Background Spin Angular Momentum

$S_{j,+0}$ : Intrinsic Spin Angular Momentum

$\Gamma_{j,+0}^{\mathbf{D}}:$ Down Background Spin Angular Momentum

\section{note}

Island operators $\Omega_{j,+0}$ possess the highest symmetry in the frame of $\mathbf{C S H}$, which can be seem through (6), (7), (8).

The principal diagonal matrix elements of $\Omega_{1,+0}^{2}(10)$ and $\Omega_{2,+0}^{2}(11)$ are the same, whereas the off-diagonal matrix elements take the contrary sign.

The values of the third component $\Omega_{3,+0}^{2}$ (13) are always to be larger than or equal to those of total square matrix $\Omega_{+0}^{2}(14)$ !

If postulating $\Omega_{+0}^{2}(14)$ to be the conservation vacuum angular momentum, what does the transitions among the different eigenvalues of $\Omega_{3,+0}$ (8) mean? 


\section{Spin $\hbar / 2$ CSH $\{3 / 4,+1 / 2,(+)\}, \Omega_{j,+1 / 2}$}

Symbol $\{3 / 4,+1 / 2,(+)\} \equiv\left\{\xi^{2}=3 / 4, n=+1 / 2, \quad(+)=n_{+}=n\right\}$

$$
\text { Here } \quad \Omega_{j,+1 / 2} \equiv \Omega_{j, n=n_{+}=+1 / 2}^{(+)=n_{+}} \equiv \xi_{j,+1 / 2}^{(+)}
$$

The following are the concrete expressions of $\Omega_{j,+1 / 2}$ base on (6-1),(6-2),(6-3) Island operator $\Omega_{1,+1 / 2}=$

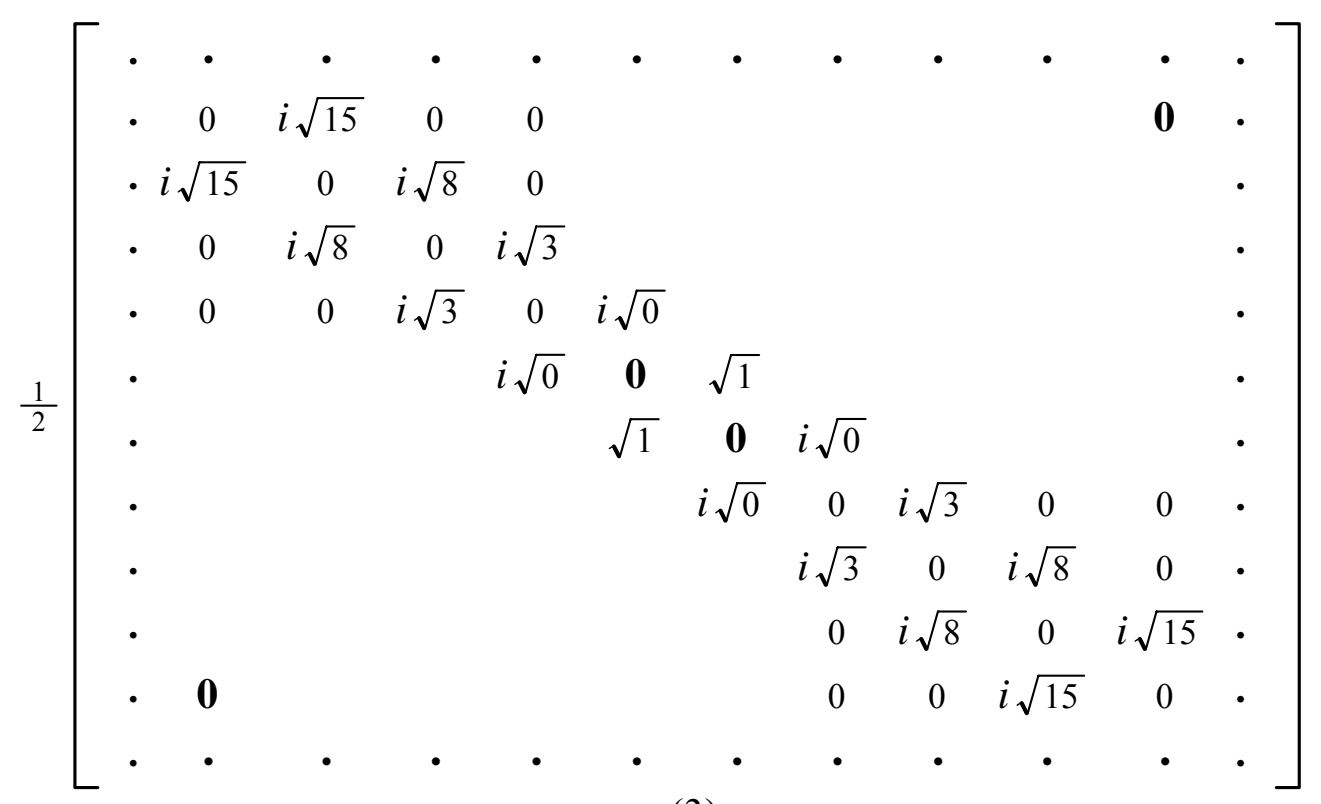

Island operator $\Omega_{2,+1 / 2}=$

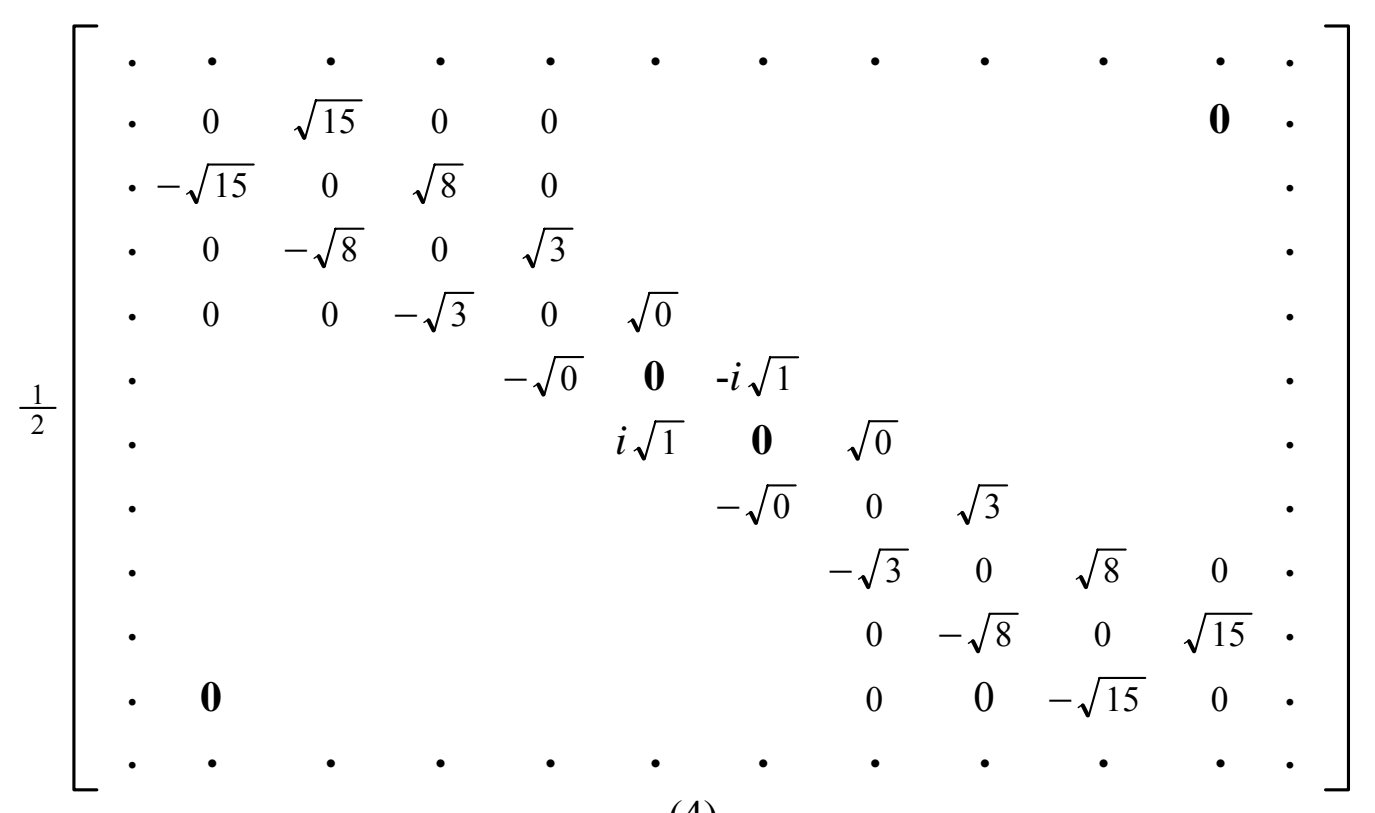

(4) 


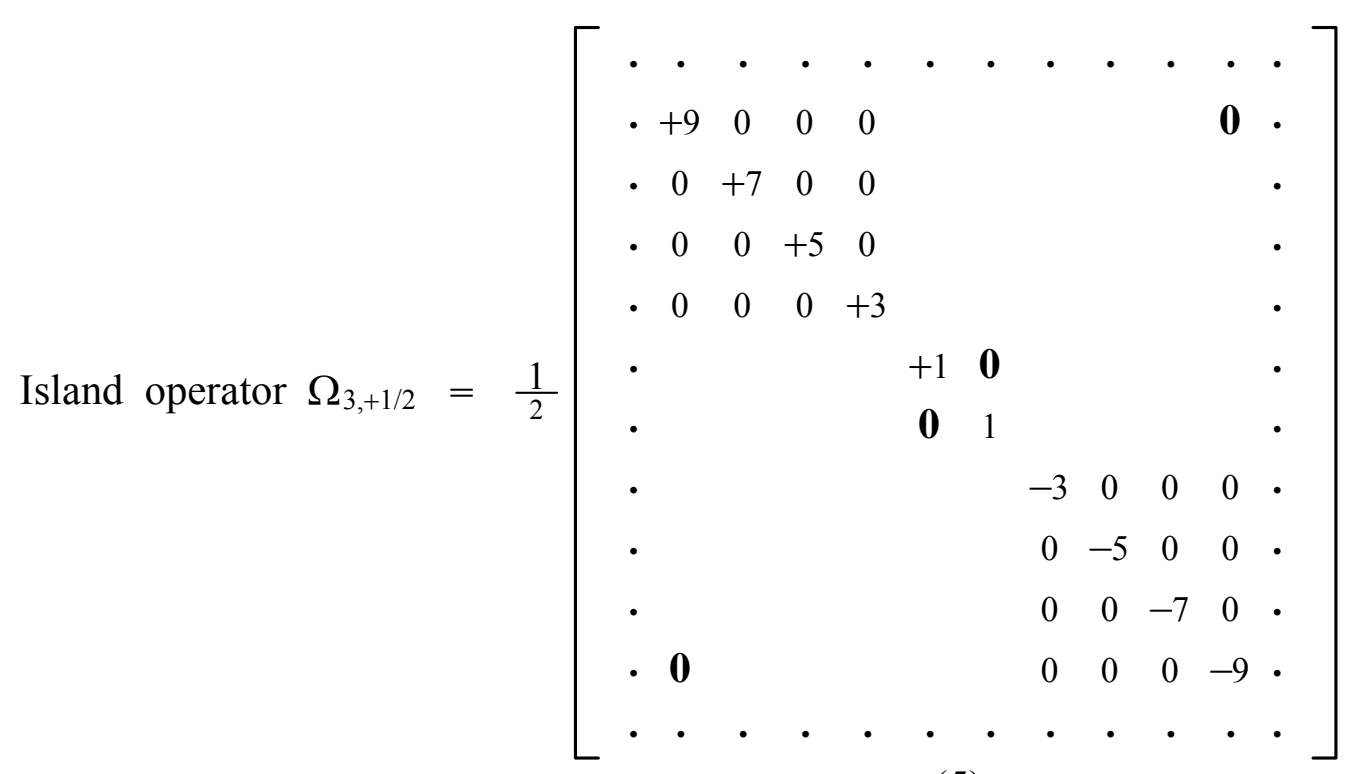

(5)

The eigenvalues, from top left to down right, of Island operators $\Omega_{3,+1 / 2}$ (5) are arranged from positive infinite to negative infinite.

(3),(4),(5) obey angular momentum commutation relation

$$
\Omega_{j,+1 / 2} \Omega_{k,+1 / 2}-\Omega_{k,+1 / 2} \Omega_{j,+1 / 2}=i \Omega_{l,+1 / 2}, j, k . l=1,2,3
$$

Evaluation of $\Omega_{1,+1 / 2}^{2}, \Omega_{2,+1 / 2}^{2}$ and $\Omega_{1,+1 / 2}^{2}+\Omega_{2,+1 / 2}^{2}$

$$
\begin{aligned}
& \Omega_{1,+1 / 2}^{2}=
\end{aligned}
$$

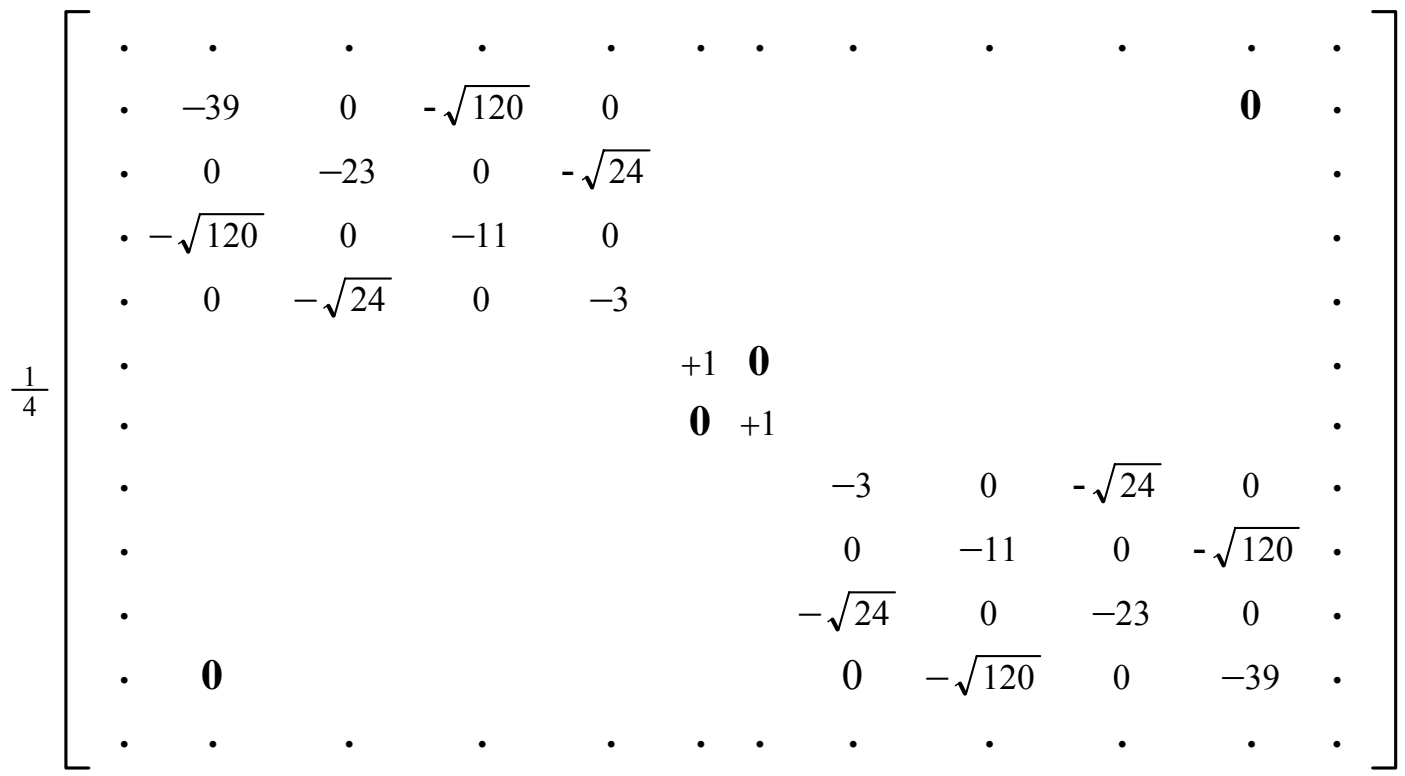




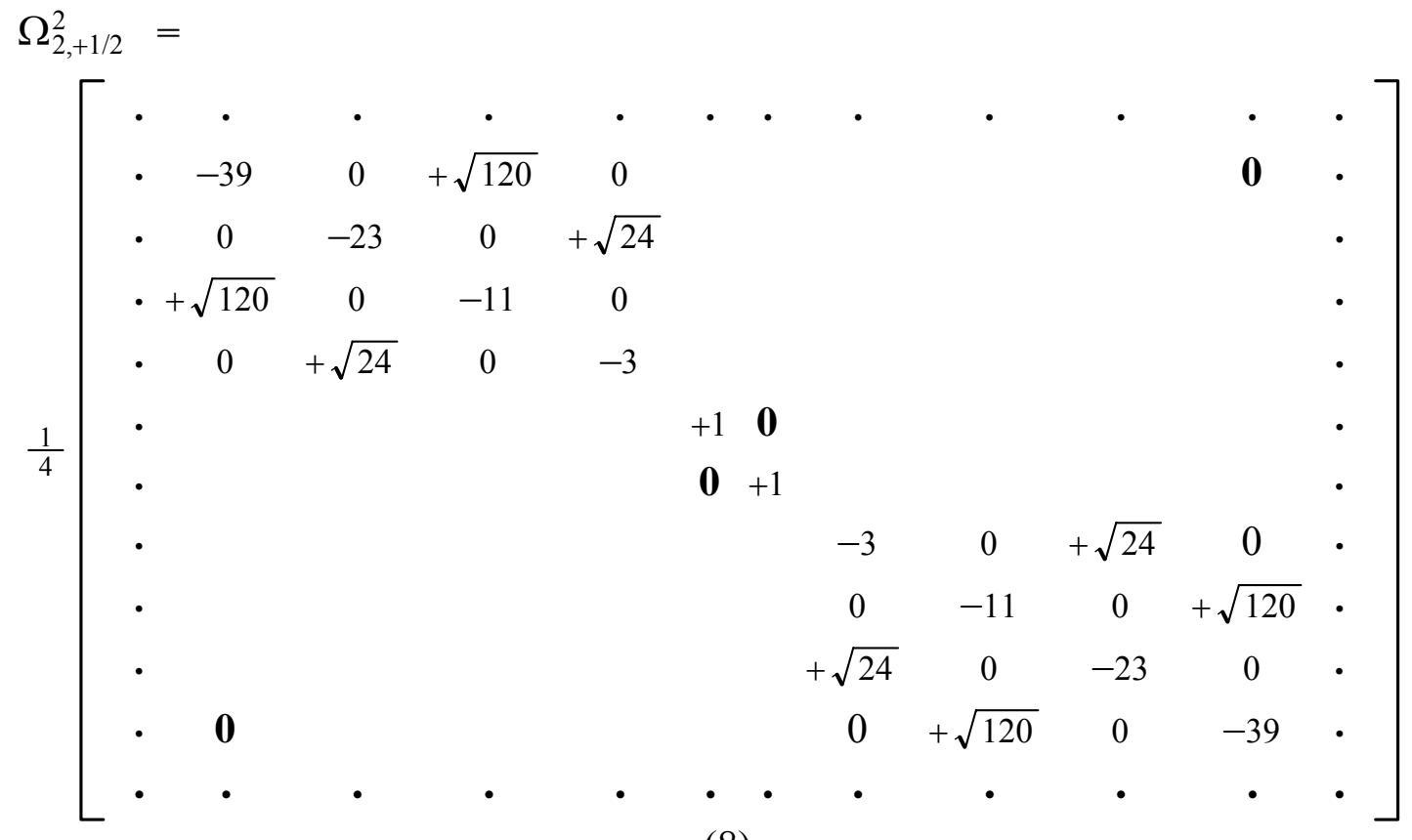

(8)

then obtain

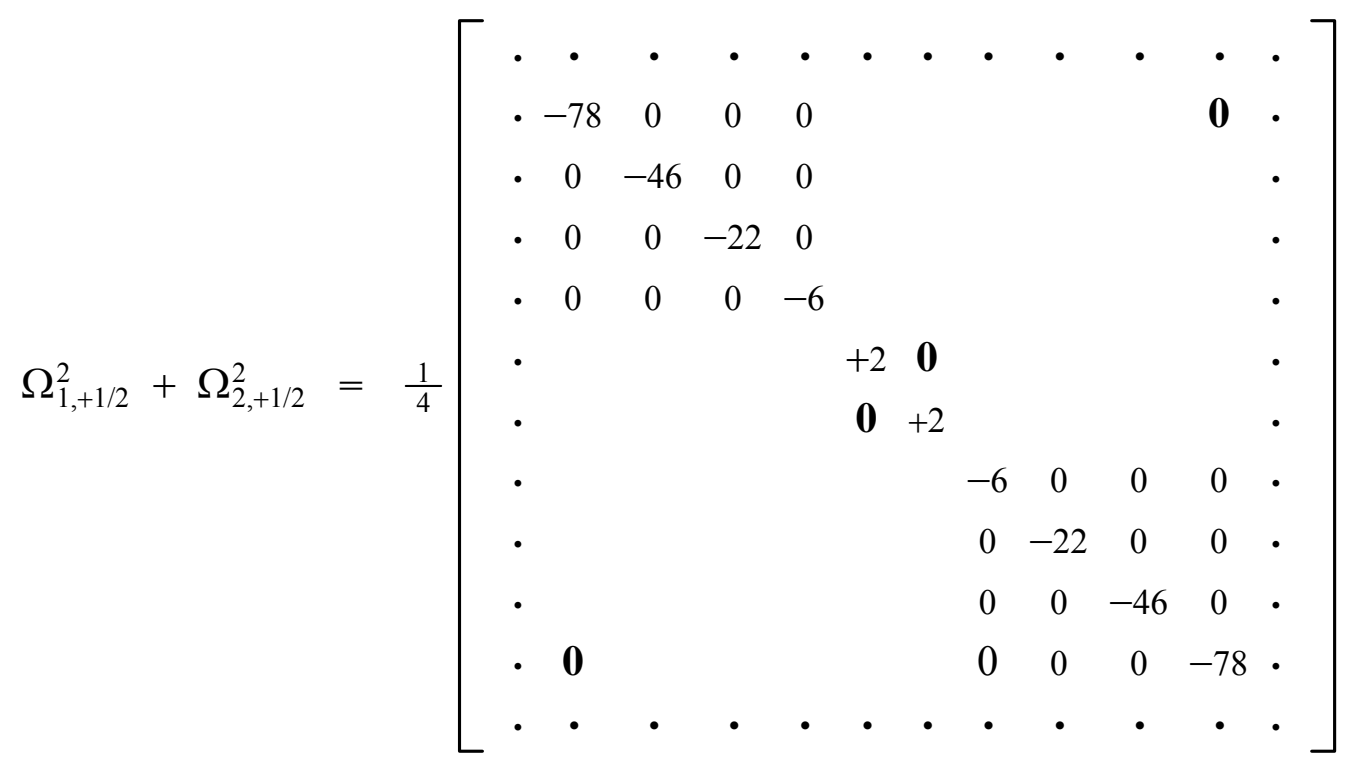




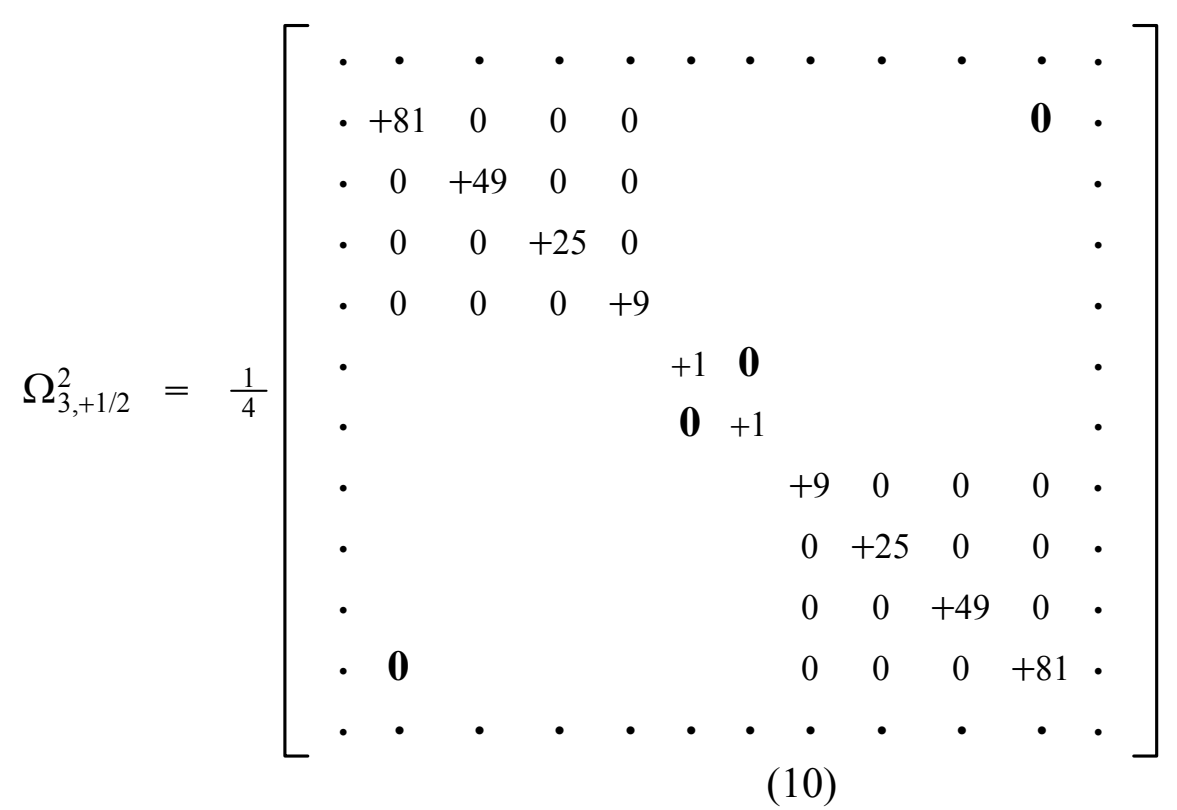

\section{note}

Although the eigenvalues of $\Omega_{1,+1 / 2}^{2}+\Omega_{2,+1 / 2}^{2}$ (9) approach to negative infinite (from the matrix center where the eigenvalues are $+1 / 2$ ) in the direction of top left and down right, the eigenvalues of $\Omega_{3}^{2}(10)$ are greater than $+1 / 4$. Further the eigenvalues of total square operator $\Omega_{+1 / 2}^{2}$ (11) remains to be a finite number $\frac{3}{4} \hbar^{2}$.

$$
\Omega_{+1 / 2}^{2}=\Omega_{1,+1 / 2}^{2}+\Omega_{2,+1 / 2}^{2}+\Omega_{3,+1 / 2}^{2}=\frac{3}{4} \hbar^{2}=\frac{1}{2}\left(\frac{1}{2} \pm 1\right) \hbar^{2} I_{0}
$$


9 Spin $1 \hbar / 3$ CSH $\{4 / 9,+1 / 3,(+)\}, \Delta_{j,+1 / 3}$

Symbol $\{4 / 9,+1 / 3,(+)\} \equiv\left\{\xi^{2}=4 / 9, n=+1 / 3, \quad(+)=n_{+}=n\right\}$

$$
\text { Here }
$$

$$
\Delta_{j,+1 / 3} \equiv \Delta_{j, n=n_{+}=+1 / 3}^{(+)=n_{+}} \equiv \xi_{j,+1 / 3}^{(+)}
$$

Ocean Operator: $\Delta_{1,+1 / 3}=\left[\begin{array}{cc}\Pi_{1,+4 / 3}^{\mathrm{U}} & 0 \\ 0 & \Pi_{1,+1 / 3}^{\mathbf{D}}\end{array}\right]=\frac{1}{\sqrt{6}} \cdot$

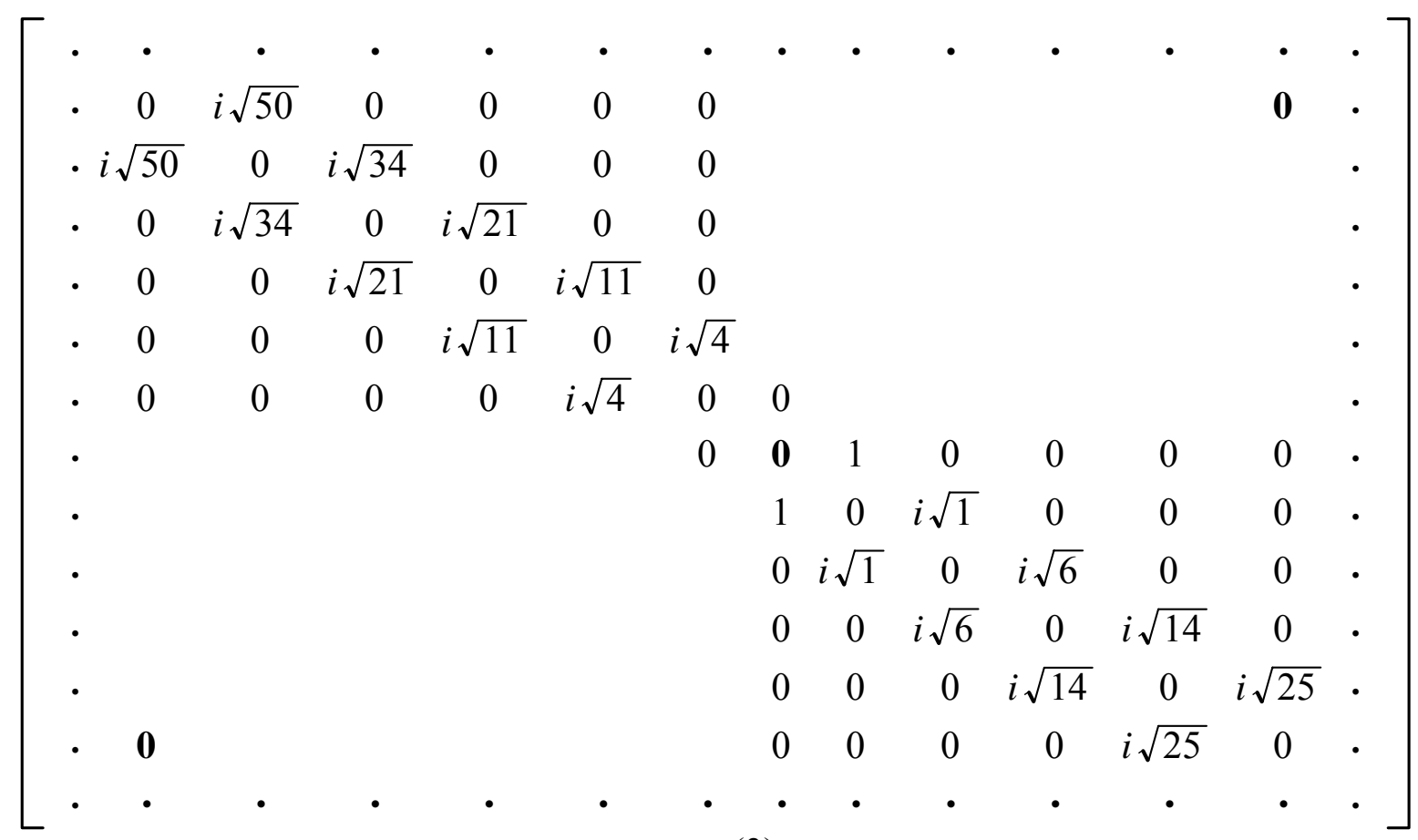

(3) 
Ocean Operator: $\Delta_{2,+1 / 3}=\left[\begin{array}{cc}\Pi_{2,+4 / 3}^{\mathbf{U}} & 0 \\ 0 & \Pi_{2,+1 / 3}^{\mathbf{D}}\end{array}\right]=\frac{1}{\sqrt{6}} \cdot$

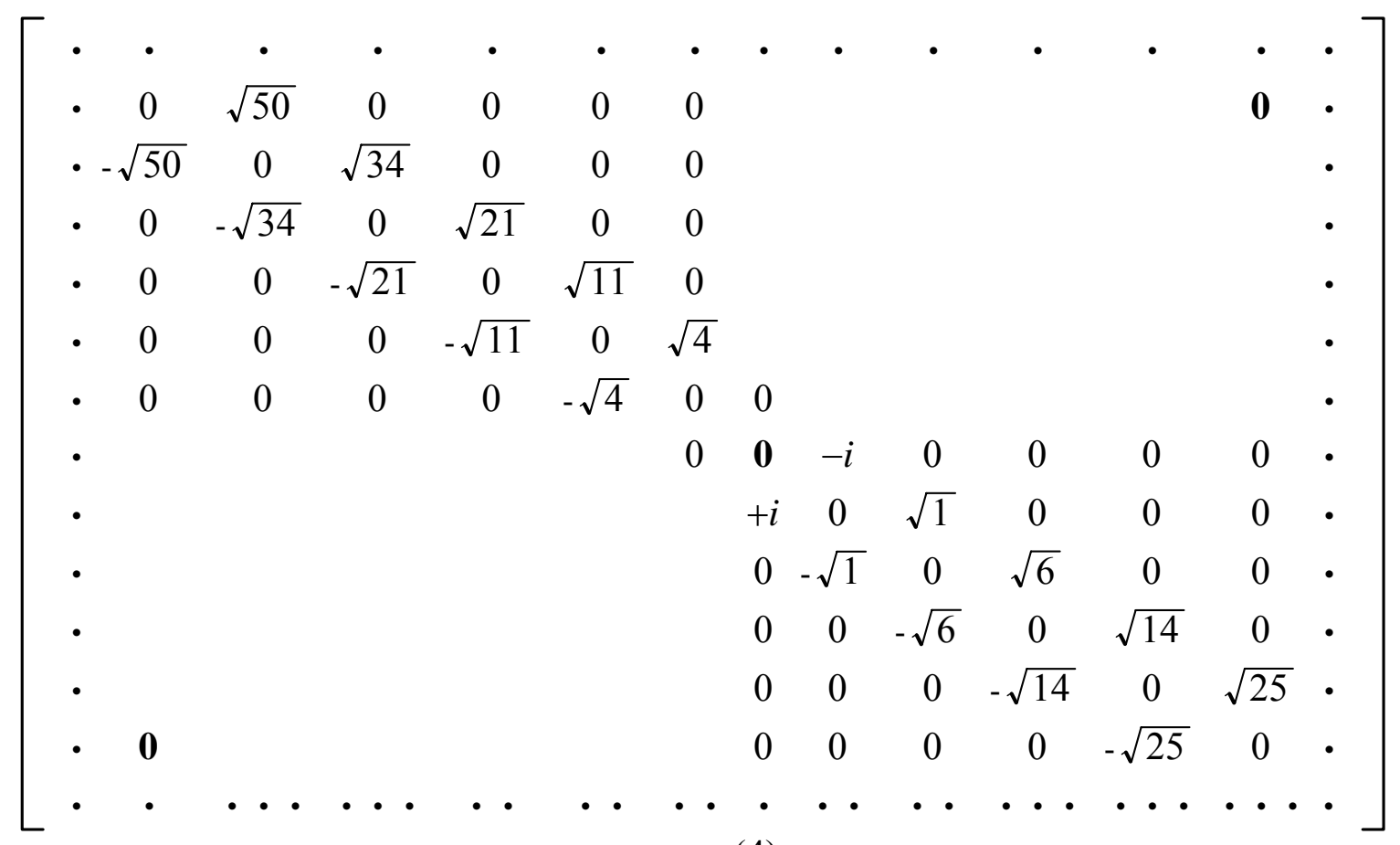

(4)

Ocean Operator: $\Delta_{3,+1 / 3}=\left[\begin{array}{cc}\Pi_{3,+4 / 3}^{\mathbf{U}} & 0 \\ 0 & \Pi_{3,+1 / 3}^{\mathbf{D}}\end{array}\right]$

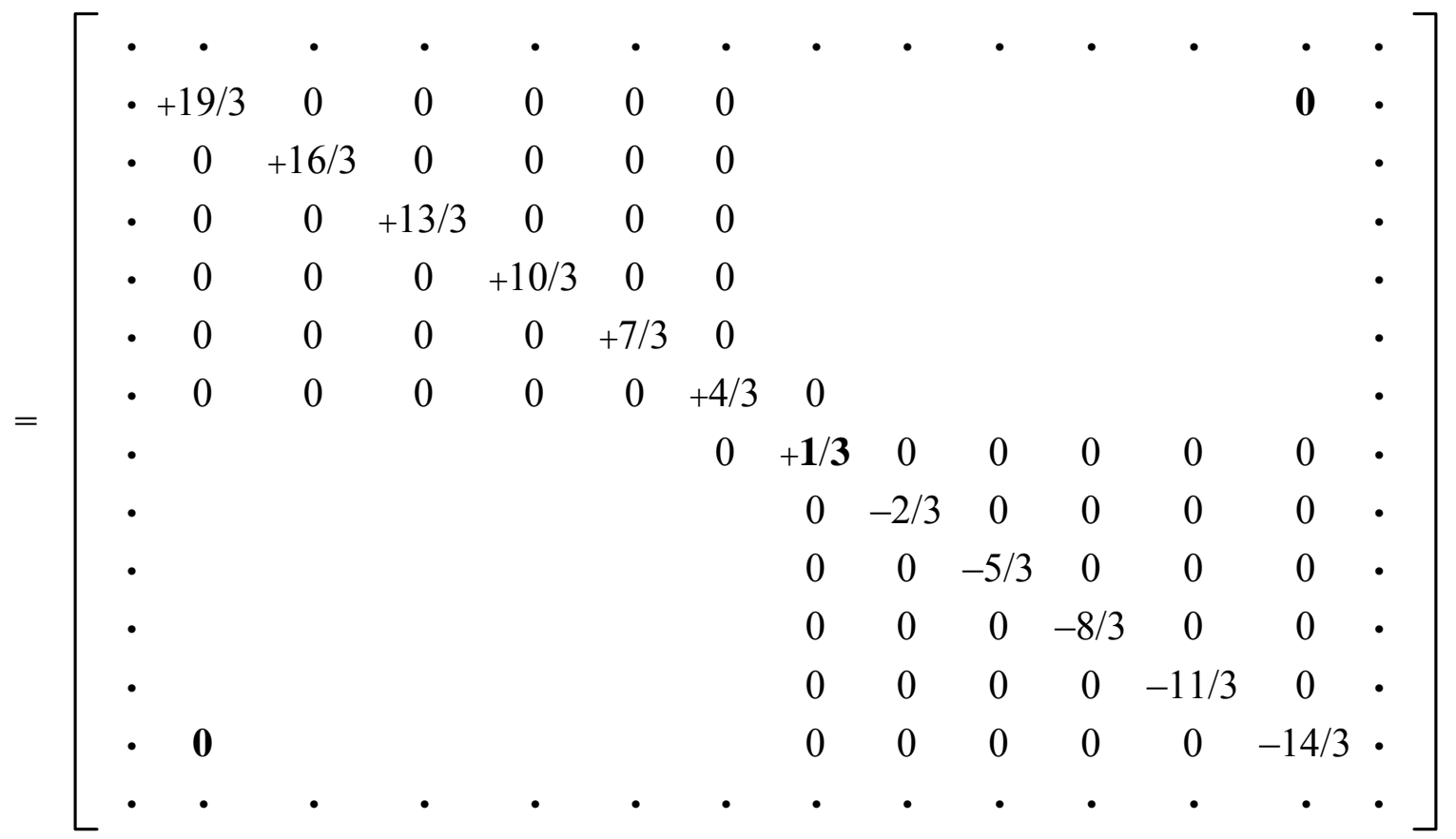

(5) 
Obtain $\Delta_{3,+1 / 3}^{2}$

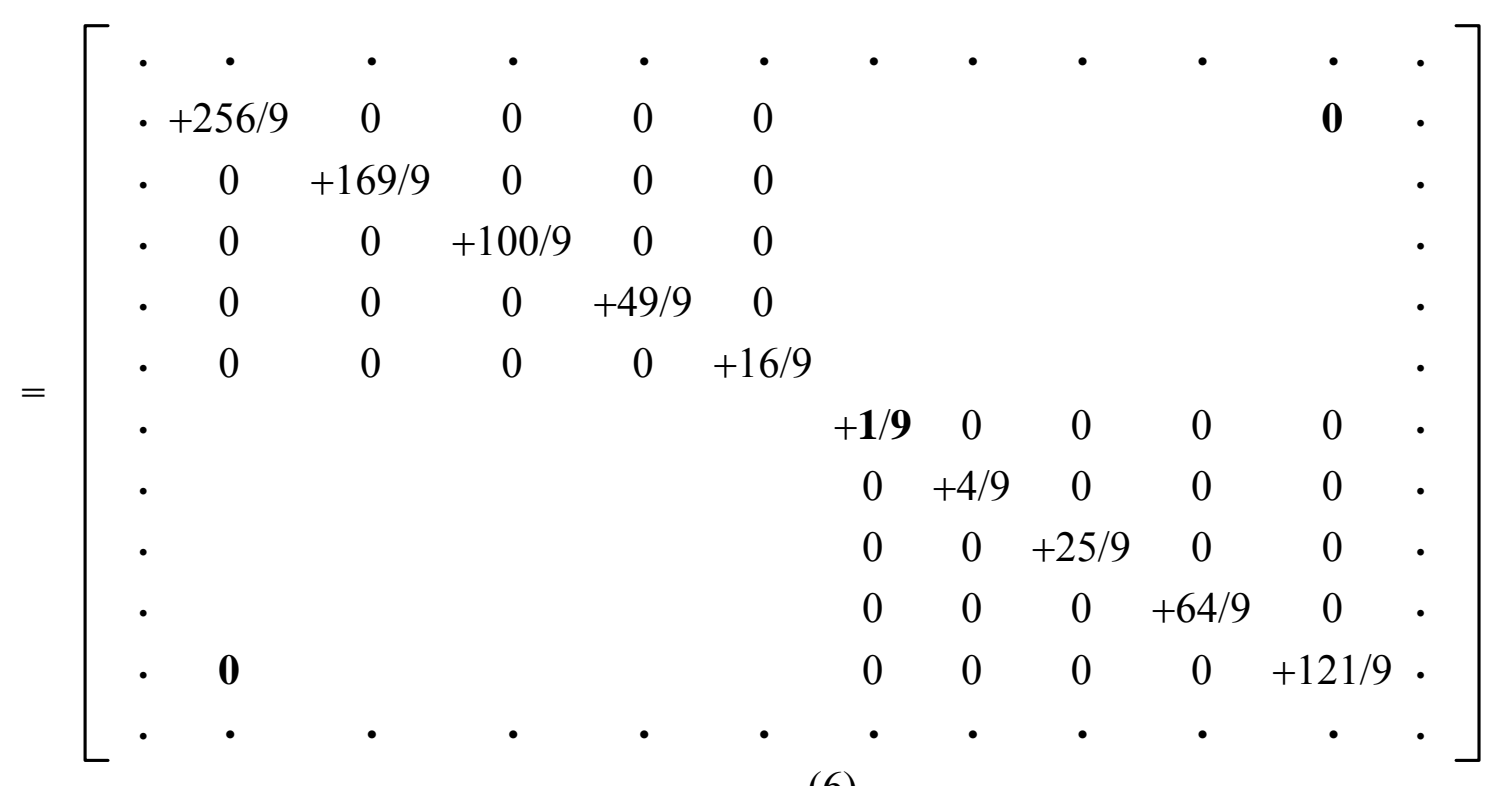

(3),(4),(5) obey angular momentum commutation relation

$$
\Delta_{j,+1 / 3}^{2} \Delta_{k,+1 / 3}^{2}-\Delta_{k,+1 / 3}^{2} \Delta_{j,+1 / 3}^{2}=i \Delta_{l,+1 / 3}^{2}, \quad j, k . l=1,2,3
$$

We have

$\Delta_{1,+1 / 3}^{2}=\frac{1}{6}$.

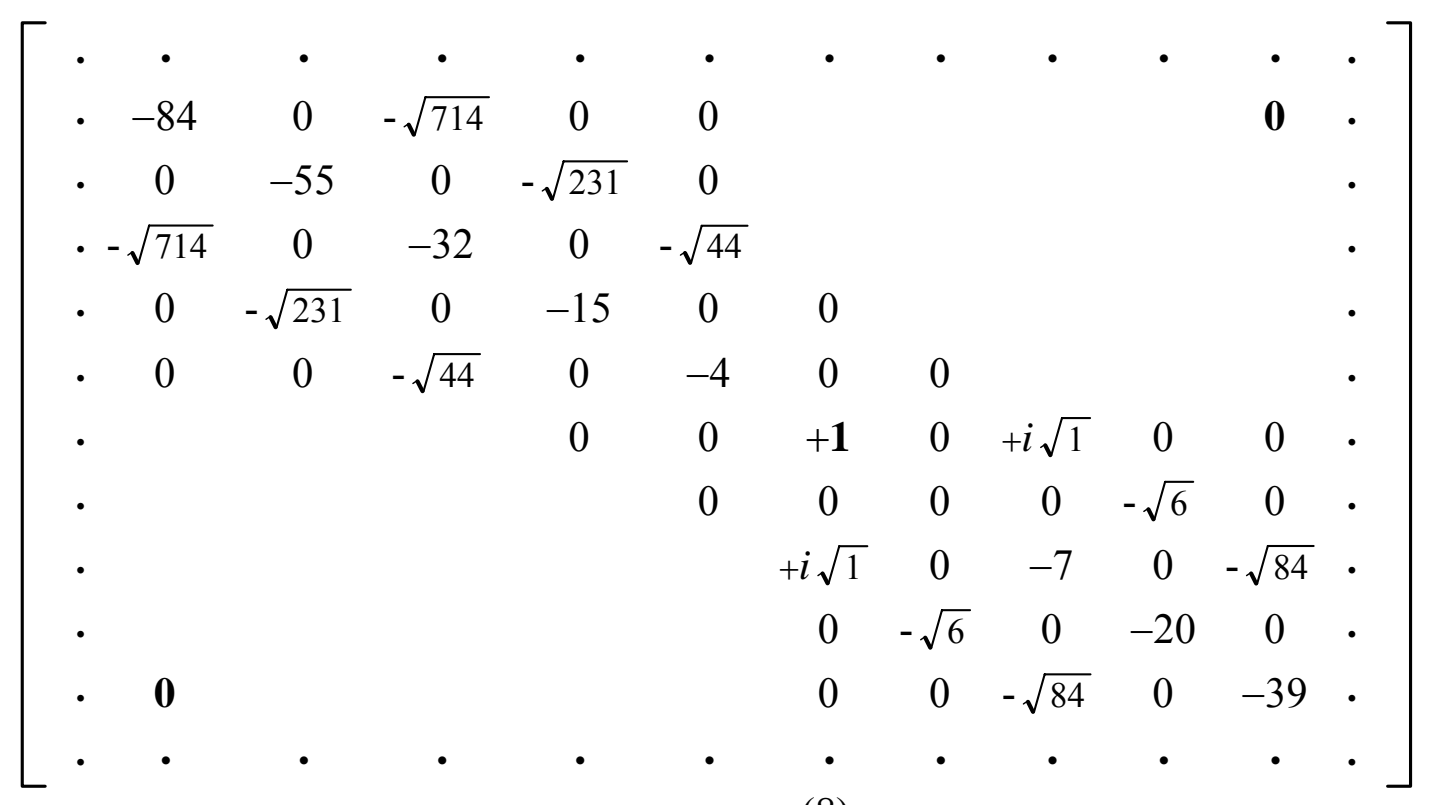

(8) 
$\Delta_{2,+1 / 3}^{2}=\frac{1}{6} \cdot$

$\left[\begin{array}{cccccccccccc}\cdot & \cdot & \cdot & \cdot & \cdot & \cdot & \cdot & \cdot & \cdot & \cdot & \cdot & \cdot \\ \cdot & -84 & 0 & +\sqrt{714} & 0 & 0 & & & & & 0 & \cdot \\ \cdot & 0 & -55 & 0 & +\sqrt{231} & 0 & & & & & & \cdot \\ \cdot+\sqrt{714} & 0 & -32 & 0 & +\sqrt{44} & & & & & & \cdot \\ \cdot & 0 & +\sqrt{231} & 0 & -15 & 0 & 0 & & & & & \cdot \\ \cdot & 0 & 0 & +\sqrt{44} & 0 & -4 & 0 & 0 & & & & \cdot \\ \cdot & & & & 0 & 0 & +\mathbf{1} & 0 & -i \sqrt{1} & 0 & 0 & \cdot \\ \cdot & & & & & 0 & 0 & 0 & 0 & +\sqrt{6} & 0 & \cdot \\ \cdot & & & & & & -i \sqrt{1} & 0 & -7 & 0 & +\sqrt{84} & \cdot \\ \cdot & & & & & & 0 & +\sqrt{6} & 0 & -20 & 0 & \cdot \\ \cdot & 0 & & & & & 0 & 0 & +\sqrt{84} & 0 & -39 & \cdot \\ \cdot & \cdot & \cdot & \cdot & \cdot & \cdot & \cdot & \cdot & \cdot & \cdot & \cdot & \cdot\end{array}\right]$

(9)

Obtain $\Delta_{1,+1 / 3}^{2}+\Delta_{2,+1 / 3}^{2}=$

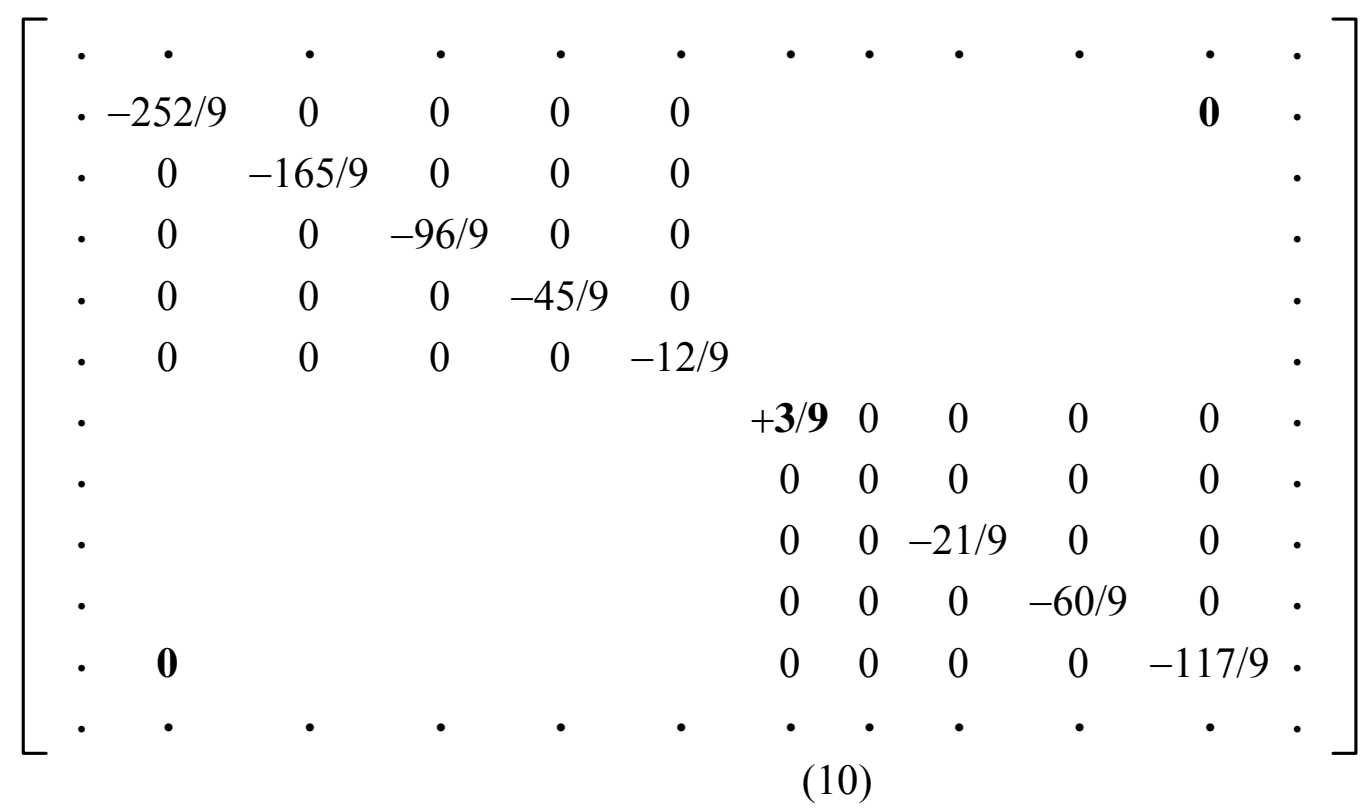

Hence

$$
\Delta_{+1 / 3}^{2}=\Delta_{1,+1 / 3}^{2}+\Delta_{2,+1 / 3}^{2}+\Delta_{3,+1 / 3}^{2}=\frac{4}{9} I_{0}=\frac{1}{3}\left(\frac{1}{3} \pm 1\right) I_{0}
$$




\section{note}

Let us go back to $\Delta_{3,+1 / 3}(5)$. first note that $\Delta_{3,+1 / 3}$ is an infinite dimensional diagonal matrix, from top left to down right, its eigenvalues are arranged from positive infinite to negative infinite. And its diagonal elements construct an arithmetic series, the difference between every two neighbour matrix elements of $\Delta_{3,+1 / 3}$ are always integral number +1 .

Each matrix element on the principal diagonal of $\Delta_{3,+1 / 3}^{2}(6)$ is the positive real number. The least value is $1 / 9$, that lies at the center of $\Delta_{3,+1 / 3}^{2}$. Upward to top left side and downward to down right side of $\Delta_{3,+1 / 3}^{2}$, the eigenvalues of $\Delta_{3,+1 / 3}^{2}$ vary toward positive infinite.

$\Delta_{1, \pm 1 / 3}^{2}$ (8) and $\Delta_{2, \pm 1 / 3}^{2}$ (9) are non-Hermitian matrices , but the sum $\Delta_{1,+1 / 3}^{2}+\Delta_{2,+1 / 3}^{2}$ (10) of them is a Hermitian diagonal matrix, except $+3 / 9$ and 0 , the rest of principal diagonal elements of (10) are all negative. Obviously, this result comes from the non-Hermiticity of matrices $\Delta_{1,+1 / 3}$ and $\Delta_{2,+1 / 3}$.

Fortunately, the increasing speed of diagonal values of matrix elements of $\Delta_{3,+1 / 3}^{2}$ (6), toward positive infinite, is slightly faster than that of $\Delta_{1,+1 / 3}^{2}+\Delta_{2,+1 / 3}^{2}$ (10) toward negative, that assures the total square spin angular momentum $\Delta_{+1 / 3}^{2}(11)$ to be a positive infinite dimensional diagonal matrix.

There are only two diagonal matrix blocks for Ocean Operator.

The difference between every two adjacent elements of main diagonal of $\Omega_{3,+1 / 3}$ (5) are always integral number 1 , to a certainty, is same as those of $\Omega_{3,+0}(7-8)$ and, $\Omega_{3,+1 / 2}(8-5)$ mentioned before. it is an essential regularity associated with CSH $\xi_{3}\left(n_{ \pm}=n\right)$. 


\section{Non-Hermitian Momentum $\overrightarrow{\mathbb{P}}$ Phase Factor of Fractional Statistics}

\subsection{Non-Hermitian Momentum $\overrightarrow{\mathbb{P}}$}

Momentum and angular momentum are the most fundamental concepts in quantum mechanics, which describe the linear motion and rotational motion of the particles in physics.

After the disscussion of positive definite non-Hermitian self-adjoint angular momentum $\overrightarrow{\mathbb{L}}$, in this paragraph we turn to positive definite non-Hermitian self-adjoint momentum $\overrightarrow{\mathbb{P}}$.

Hermitian Momentum $\vec{P}$ is defined as

$$
\begin{aligned}
& P_{1}=-i \sin \theta \cos \varphi \partial_{r}-i \frac{1}{r} \cos \theta \cos \varphi \partial_{\theta}+i \frac{\sin \varphi}{r \sin \theta} \partial_{\varphi} \\
& P_{2}=-i \sin \theta \sin \varphi \partial_{r}-i \frac{1}{r} \cos \theta \sin \varphi \partial_{\theta}-i \frac{\cos \varphi}{r \sin \theta} \partial_{\varphi} \\
& P_{3}=-i \cos \theta \partial_{r}+i \frac{1}{r} \sin \theta \partial_{\theta}
\end{aligned}
$$

In spherical coordinates, we have the radial metric coefficient $\mathrm{f}(r)=r^{2}$, then the total metric coefficient $\alpha$ of space is extended to three coordindate functions as follows (2)

$$
\alpha=\mathrm{f}(r) \mathrm{g}(\theta) \mathrm{h}(\varphi) ; \quad \mathrm{f}(r)=r^{2}, \quad \mathrm{~g}(\theta)=\sin ^{1+4 m_{0}} \theta, \quad \mathrm{h}(\varphi)=\kappa+2 \eta \mathbb{T}_{1}
$$

Then take the Positive Definite Non-Hermitian Adjoint Operation of momentum (1.1),(1.2),(1.3)

$$
\begin{aligned}
& \left(P_{1}\right)^{\oplus}=+i \sin \theta \cos \varphi \partial_{r}^{\oplus}+i \frac{1}{r} \cos \varphi \partial_{\theta}^{\oplus} \cos \theta-i \frac{1}{r \sin \theta} \partial_{\varphi}^{\oplus} \sin \varphi \\
& \left(P_{2}\right)^{\oplus}=+i \sin \theta \sin \varphi \partial_{r}^{\oplus}+i \frac{1}{r} \sin \varphi \partial_{\theta}^{\oplus} \cos \theta+\frac{1}{r \sin \theta} \partial_{\varphi}^{\oplus} \cos \varphi \\
& \left(P_{3}\right)^{\oplus}=+i \cos \theta \partial_{r}^{\oplus}-i \frac{1}{r} \partial_{\theta}^{\oplus} \sin \theta
\end{aligned}
$$

Substitution of (2) into the adjoint representation of derivative operator (1-21) separately, yields the adjoint representins of derivative operator $\partial_{r}, \partial_{\theta}, \partial_{\varphi}$ as follows

$$
\begin{aligned}
& \partial_{r}^{\oplus}=-\partial_{r}-\frac{2}{r} \\
& \partial_{\theta}^{\oplus}=-\partial_{\theta}-\left(1+4 m_{0}\right) \cot \theta \\
& \partial_{\varphi}^{\oplus}=-\partial_{\varphi}-2 \eta \mathbb{T}_{2}
\end{aligned}
$$


On using the (4),(5),(6), therefore

$$
\begin{aligned}
& \left(P_{1}\right)^{\oplus}=-i \sin \theta \cos \varphi \partial_{r}-i \frac{1}{r} \cos \theta \cos \varphi\left(\partial_{\theta}+4 m_{0} \cot \theta\right)-\frac{\sin \varphi}{r \sin \theta}\left(l_{3}-i 2 \eta \mathbb{T}_{2}\right) \\
& \left(P_{2}\right)^{\oplus}=-i \sin \theta \sin \varphi \partial_{r}-i \frac{1}{r} \cos \theta \sin \varphi\left(\partial_{\theta}+4 m_{0} \cot \theta\right)+\frac{\cos \varphi}{r \sin \theta}\left(l_{3}-i 2 \eta \mathbb{T}_{2}\right) \\
& \left(P_{3}\right)^{\oplus}=-i \cos \theta \partial_{r}+i \frac{1}{r} \sin \theta\left(\partial_{\theta}+4 m_{0} \cot \theta\right)
\end{aligned}
$$

Because of

$$
\begin{aligned}
& \left(P_{1}\right)^{\oplus} \neq P_{1} \\
& \left(P_{2}\right)^{\oplus} \neq P_{2} \\
& \left(P_{3}\right)^{\oplus} \neq P_{3}
\end{aligned}
$$

Consequently, the so-called well-definited momentum operator in space (2), the positive definite non-Hermitian self-adjoint operator $\overrightarrow{\mathbb{P}}$ is introduced by following definition

$$
\overrightarrow{\mathbb{P}}=\frac{1}{2}\left\{(\vec{P})^{\oplus}+\vec{P}\right\}
$$

Then we have

$$
\begin{aligned}
& \mathbb{P}_{1}=-i \sin \theta \cos \varphi \partial_{r}-i \frac{1}{r} \cos \theta \cos \varphi\left(\partial_{\theta}+2 m_{0} \cot \theta\right)-\frac{1}{r} \frac{\sin \varphi}{\sin \theta} \mathbb{L}_{3} \\
& \mathbb{P}_{2}=-i \sin \theta \sin \varphi \partial_{r}-i \frac{1}{r} \cos \theta \sin \varphi\left(\partial_{\theta}+2 m_{0} \cot \theta\right)+\frac{1}{r} \frac{\cos \varphi}{\sin \theta} \mathbb{L}_{3} \\
& \mathbb{P}_{3}=-i \cos \theta \partial_{r}+i \frac{1}{r} \sin \theta\left(\partial_{\theta}+2 m_{0} \cot \theta\right)
\end{aligned}
$$

After carefully evaluation, momentum square operator is written by

$$
\mathbb{P}^{2}=\mathbb{P}_{1}^{2}+\mathbb{P}_{2}^{2}+\mathbb{P}_{3}^{2}=P_{r}^{2}+\frac{1}{r^{2}} \mathbb{L}^{2}
$$

Note where

$$
\begin{aligned}
P_{r} & =-i \partial_{r}-i \frac{1}{r} \\
\mathbb{L}^{2} & =-\left\{\partial_{\theta}^{2}+\left(1+4 m_{0}\right) \cot \theta \partial_{\theta}-(\sin \theta)^{-2}\left(\mathbb{L}_{3}^{2}-4 m_{0}^{2}\right)-4 m_{0}^{2}-2 m_{0}\right\}
\end{aligned}
$$

Here $\mathbb{L}^{2}$ is what we have obtained in (2-19) namely, non-Heemitian orbital angular momentum square operator.

10.2 By means of the orthonormal bases $\vec{e}_{r}, \vec{e}_{\theta}, \vec{e}_{\varphi}$ in spherical coordinates

$$
\vec{e}_{r}=\left(\begin{array}{c}
\sin \theta \cos \varphi \\
\sin \theta \sin \varphi \\
\cos \theta
\end{array}\right), \vec{e}_{\theta}=\left(\begin{array}{c}
\cos \theta \cos \varphi \\
\cos \theta \sin \varphi \\
-\sin \theta
\end{array}\right), \quad \vec{e}_{\varphi}=\left(\begin{array}{c}
-\sin \varphi \\
+\cos \varphi \\
0
\end{array}\right)
$$


Using (14) to rewrite (10.1),(10.2),(10.3), we have (15)

So

$$
\overrightarrow{\mathbb{P}}=-i \vec{e}_{r} \partial_{r}-i \frac{1}{r} \vec{e}_{\theta}\left(\partial_{\theta}+2 m_{0} \cot \theta\right)+\frac{1}{r \sin \theta} \vec{e}_{\varphi} \mathbb{L}_{3}
$$

Position operator $\vec{r}$

$$
\vec{r}=r \vec{e}
$$

On account of basis rules

$$
\begin{aligned}
\vec{e}_{r} \times \vec{e}_{\theta} & =\vec{e}_{\varphi}, \vec{e}_{\theta} \times \vec{e}_{\varphi}=\vec{e}_{r}, \vec{e}_{\varphi} \times \vec{e}_{r}=\vec{e}_{\theta} \\
\left(\vec{e}_{r}\right)^{2} & =\left(\vec{e}_{\theta}\right)^{2}=\left(\vec{e}_{r}\right)^{2}=1
\end{aligned}
$$

We conclude cross product $\vec{r} \times \overrightarrow{\mathbb{P}}$ of position operator $\vec{r}$ (16) with momentum operator $\overrightarrow{\mathbb{P}}(15)$, obtain

$$
\begin{aligned}
& \vec{r} \times \overrightarrow{\mathbb{P}} \\
= & r \vec{e}_{r} \times\left\{-i \vec{e}_{r} \partial_{r}-i \frac{1}{r} \vec{e}_{\theta}\left(\partial_{\theta}+2 m_{0} \cot \theta\right)+\frac{1}{r \sin \theta} \vec{e}_{\varphi} \mathbb{L}_{3}\right\} \\
= & 0-i \frac{1}{r} \vec{e}_{\varphi}\left(\partial_{\theta}+2 m_{0} \cot \theta\right)-\frac{1}{\sin \theta} \vec{e}_{\theta} \mathbb{L}_{3}
\end{aligned}
$$

and three components of (18) are

$$
\begin{aligned}
& (\vec{r} \times \overrightarrow{\mathbb{P}})_{1}=+i \sin \varphi\left(\partial_{\theta}+2 m_{0} \cot \theta\right)-\cot \theta \cos \varphi \mathbb{L}_{3} \\
& (\vec{r} \times \overrightarrow{\mathbb{P}})_{2}=-i \cos \varphi\left(\partial_{\theta}+2 m_{0} \cot \theta\right)-\cot \theta \sin \varphi \mathbb{L}_{3} \\
& (\vec{r} \times \overrightarrow{\mathbb{P}})_{3}=-i \partial_{\varphi}-i \eta \mathbb{T}_{2}
\end{aligned}
$$

Comparing (20.1), (20.2), (20.3) with (2-13), (2-14), (2-15), we see that the definition $\vec{r} \times \overrightarrow{\mathbb{P}}(18)$ is namely positive definite non-Hermitian self-adjoint orbital angular momentum previously obtained.

$$
\begin{aligned}
\vec{r} \times \overrightarrow{\mathbb{P}} & \equiv \overrightarrow{\mathbb{L}} \\
\overrightarrow{\mathbb{L}} & =-i\left(\begin{array}{c}
-\sin \varphi \\
+\cos \varphi \\
0
\end{array}\right)\left(\partial_{\theta}+2 m_{0} \cot \theta\right)-\frac{1}{\sin \theta}\left(\begin{array}{c}
\cos \theta \cos \varphi \\
\cos \theta \sin \varphi \\
-\sin \theta
\end{array}\right) \mathbb{L}_{3}
\end{aligned}
$$

Next we will use non-Hermitian momentum operator $\overrightarrow{\mathbb{P}}$ to give some interesting and heuristic ideas which related to gauge invariance in $\alpha$ space (2) and the phase factor of fractional statistics of particle wavefunctions. 
10.3 The Gauge Invariance in Space $\mathbf{f}(r) \mathbf{g}(\theta) \mathbf{h}(\varphi)$

a) Non-Hermitian momentum $\overrightarrow{\mathbb{P}}(15)$ can be expressed by derivative $\vec{\mho}$.

$$
\overrightarrow{\mathbb{P}}=-i \vec{\zeta}
$$

Where

$$
\begin{aligned}
\vec{\mho} & =\vec{e}_{r} \partial_{r}+\frac{1}{r} \vec{e}_{\theta}\left(\partial_{\theta}+2 m_{0} \cot \theta\right)+i \frac{1}{r \sin \theta} \vec{e}_{\varphi} \mathbb{L}_{3} \\
& =\vec{\partial}+\frac{1}{r} \vec{e}_{\theta} 2 m_{0} \cot \theta+\frac{1}{r \sin \theta} \vec{e}_{\varphi} \eta \mathbb{T}_{2}(\varphi) \\
\vec{\mho} & =\vec{\partial}+\vec{\triangle}
\end{aligned}
$$

Where

$$
\begin{aligned}
& \vec{\partial}=-i \vec{e}_{r} \partial_{r}-i \frac{1}{r} \vec{e}_{\theta} \partial_{\theta}+\frac{1}{r \sin \theta} \vec{e}_{\varphi} l_{3} \\
& \vec{\triangle}=\frac{1}{r} \vec{e}_{\theta} 2 m_{0} \cot \theta+\frac{1}{r \sin \theta} \vec{e}_{\varphi} \eta \mathbb{T}_{2}(\varphi)
\end{aligned}
$$

If no confusion, we use symbol $\vec{\nabla}=\vec{\partial}$. The components of $\vec{\triangle}$ as follows

$$
\begin{aligned}
\triangle_{r} & =0, \quad \triangle_{\theta}=2 m_{0} \frac{1}{r} \cot \theta, \quad \triangle_{\varphi}=\eta \frac{1}{r \sin \theta} \mathbb{T}_{2}(\varphi) \\
\sin \theta \triangle_{\varphi} & =\frac{1}{r} \eta \mathbb{T}_{2}(\varphi) \\
r \triangle_{\varphi} & =\frac{1}{\sin \theta} \eta \mathbb{T}_{2}(\varphi) \\
r \triangle_{\theta} & =2 m_{0} \cot \theta
\end{aligned}
$$

Using the above results to evaluate $\vec{\nabla} \times \vec{\triangle}$, obtaim

$$
\begin{aligned}
\vec{\nabla} \times \vec{\triangle}= & \frac{1}{r \sin \theta}\left[\partial_{\theta}\left(\sin \theta \triangle_{\varphi}\right)-\partial_{\varphi}\left(\triangle_{\theta}\right)\right] \vec{e}_{r} \\
& +\frac{1}{r}\left[\frac{1}{\sin \theta} \partial_{\varphi}\left(\triangle_{r}\right)-\partial_{r}\left(r \triangle_{\varphi}\right)\right] \vec{e}_{\theta} \\
& +\frac{1}{r}\left[\partial_{r}\left(r \triangle_{\theta}\right)-\partial_{\theta}\left(\triangle_{r}\right)\right] \vec{e}_{\varphi} \\
= & \frac{1}{r \sin \theta}\left[\partial_{\theta}\left(\frac{1}{r} \eta \mathbb{T}_{2}(\varphi)-\partial_{\varphi}\left(2 m_{0} \frac{1}{r} \cot \theta\right)\right] \vec{e}_{r}\right. \\
& +\frac{1}{r}\left[\frac{1}{\sin \theta} \partial_{\varphi}(0)-\partial_{r}\left(\frac{1}{\sin \theta} \eta \mathbb{T}_{2}(\varphi)\right)\right] \vec{e}_{\theta} \\
& +\frac{1}{r}\left[\partial_{r}\left(2 m_{0} \cot \theta\right)-\partial_{\theta}(0)\right] \vec{e}_{\varphi} \\
= & 0
\end{aligned}
$$

further

$$
\begin{aligned}
& \vec{\nabla} \times \vec{\triangle}=0 \\
& \vec{\mho} \times \vec{\triangle}=(\vec{\nabla}+\vec{\triangle}) \times \vec{\triangle}=0
\end{aligned}
$$

(31) and (32) show that $\vec{\triangle}$ is irrotational field respect to $\vec{\nabla}$ and derivative $\vec{\mho}$. 
b) In space (2), Schrödinger equation and minimal coupling theorem are defined

$$
\left\{\left(\overrightarrow{\mathbb{P}}-\frac{\mathrm{e}}{\mathrm{c}} \overrightarrow{\mathbb{A}}\right)^{2}+e \phi\right\} \Psi=i \hbar \partial_{t} \Psi
$$

Using (25), have

$$
\begin{aligned}
\left\{-\frac{\hbar^{2}}{2 \mathrm{~m}}\left(\vec{\mho}-i \frac{\mathrm{e}}{\hbar \mathrm{c}} \overrightarrow{\mathbb{A}}\right)^{2}+e \phi\right\} \Psi & =i \hbar \partial_{t} \Psi \\
\left\{-\frac{\hbar^{2}}{2 \mathrm{~m}}(\overrightarrow{\mathbb{D}})^{2}+e \phi\right\} \Psi & =i \hbar \partial_{t} \Psi
\end{aligned}
$$

where

$$
\overrightarrow{\mathbb{D}} \equiv \vec{\mho}-i \frac{\mathrm{e}}{\hbar \mathrm{c}} \overrightarrow{\mathbb{A}}
$$

$\overrightarrow{\mathbb{D}}$ is called the covariant derivative of wave function $\Psi$ respect to the gauge vector potential $\overrightarrow{\mathbb{A}}$ in space (2).

By means of gauge transformation (37),(38)

$$
\begin{aligned}
\Psi(x) & \Rightarrow \Psi^{\prime}(x)=\exp \left[i \frac{\mathrm{e}}{\hbar \mathrm{c}} \lambda(x)\right] \Psi(x) \\
\mathbb{A}_{j}(x) \Rightarrow \mathbb{A}_{j}^{\prime}(x) & =\mathbb{A}_{j}(x)+\left[\partial_{j} \lambda(x)\right]
\end{aligned}
$$

here $i \frac{\mathrm{e}}{\hbar c} \lambda(x)$ is phase factor of wave function $\Psi(x)$ as follow

$$
\lambda(x)=\int_{x_{0}[C]}^{x} \overrightarrow{\mathbb{A}} \cdot \overrightarrow{D l}
$$

where $\overrightarrow{D l}$ is differential length [cf.(60)]

We make following gauge transformations of covariant derivative $\mathbb{D}_{j}$ and wavefunction $\Psi$.

$$
\begin{aligned}
\mathbb{D}_{j} \Psi(x) & =\left\{\mho_{j}-i \frac{\mathrm{e}}{\hbar \mathrm{c}} \mathbb{A}_{j}\right\} \Psi(x)=\left\{\partial_{j}+\triangle_{j}-i \frac{\mathrm{e}}{\hbar \mathrm{c}} \mathbb{A}_{j}\right\} \Psi(x) \\
& \Downarrow \\
\mathbb{D}_{j}^{\prime} \Psi^{\prime}(\vec{x}) & =\left\{\mho_{j}-i \frac{\mathrm{e}}{\hbar \mathrm{c}}\left(\mathbb{A}_{j}(x)+\left[\partial_{j} \lambda(x)\right]\right)\right\} \cdot \exp \left[i \frac{\mathrm{e}}{\hbar \mathrm{c}} \lambda(x)\right] \Psi(x) \\
& =\left\{\partial_{j}+\triangle_{j}-i \frac{\mathrm{e}}{\hbar \mathrm{c}}\left(\mathbb{A}_{j}(x)+\left[\partial_{j} \lambda(x)\right]\right)\right\} \cdot \exp \left[i \frac{\mathrm{e}}{\hbar \mathrm{c}} \lambda(x)\right] \Psi(x) \\
& =\exp \left[i \frac{\mathrm{e}}{\hbar \mathrm{c}} \lambda(x)\right]\left\{\frac{\partial_{j}+i \frac{\mathrm{e}}{\hbar \mathrm{c}}\left[\partial_{j} \lambda(x)\right]}{+\triangle_{j}-i \frac{\mathrm{e}}{\hbar \mathrm{c}}\left(\mathbb{A}_{j}(x)+\left[\partial_{j} \lambda(x)\right]\right\} \Psi(x)}\right. \\
& =\exp \left[i \frac{\mathrm{e}}{\hbar \mathrm{c}} \lambda(x)\right]\left\{\partial+\Delta-i \frac{\mathrm{e}}{\hbar \mathrm{c}} \mathbb{A}_{j}(x)\right\} \Psi(x) \\
& =\exp \left[i \frac{\mathrm{e}}{\hbar \mathrm{c}} \lambda(x)\right]\left\{\mho_{j}-i \frac{\mathrm{e}}{\hbar \mathrm{c}} \mathbb{A}_{j}(x)\right\} \Psi(x) \\
\mathbb{D}_{j}^{\prime} \Psi^{\prime}(x) & =\exp \left[i \frac{\mathrm{e}}{\hbar \mathrm{c}} \lambda(x)\right]\left\{\mho_{j}-i \frac{\mathrm{e}}{\hbar \mathrm{c}} \mathbb{A}_{j}\right\} \Psi(x) \\
\mathbb{D}_{j}^{\prime} \Psi^{\prime}(x) & =\exp \left[i \frac{\mathrm{e}}{\hbar \mathrm{c}} \lambda(x)\right] \mathbb{D}_{j} \Psi(x)
\end{aligned}
$$


Using (41),(42), Further

$$
\begin{aligned}
& \mathbb{D}_{j}^{2} \Psi(x)=\left\{\mho_{j}-i \frac{\mathrm{e}}{\hbar \mathrm{c}} \mathbb{A}_{j}\right\}^{2} \Psi(x)=\left\{\partial_{j}+\Delta_{j}-i \frac{\mathrm{e}}{\hbar \mathrm{c}} \mathbb{A}_{j}\right\}^{2} \Psi(x) \\
& \Downarrow \\
& \mathbb{D}_{j}^{\prime 2} \Psi^{\prime}(x)=\left\{\mho_{j}-i \frac{\mathrm{e}}{\hbar \mathrm{c}}\left(\mathbb{A}_{j}(x)+\left[\partial_{j} \lambda(x)\right]\right)\right\}^{2} \cdot \exp \left[i \frac{\mathrm{e}}{\hbar \mathrm{c}} \lambda(x)\right] \Psi(x) \\
& =\left\{\partial_{j}+\triangle_{j}-i \frac{\mathrm{e}}{\hbar \mathrm{c}}\left(\mathbb{A}_{j}(x)+\left[\partial_{j} \lambda(x)\right]\right)\right\}^{2} \cdot \exp \left[i \frac{\mathrm{e}}{\hbar \mathrm{c}} \lambda(x)\right] \Psi(x) \\
& =\left\{\partial_{j}+\triangle_{j}-i \frac{\mathrm{e}}{\hbar \mathrm{c}}\left(\mathbb{A}_{j}(x)+\left[\partial_{j} \lambda(x)\right]\right)\right\} \cdot \\
& \left\{\partial_{j}+\triangle_{j}-i \frac{\mathrm{e}}{\hbar \mathrm{c}}\left(\mathbb{A}_{j}(x)+\left[\partial_{j} \lambda(x)\right]\right)\right\} \cdot \exp \left[i \frac{\mathrm{e}}{\hbar \mathrm{c}} \lambda(x)\right] \Psi(x) \\
& =\left\{\partial_{j}+\triangle_{j}-i \frac{\mathrm{e}}{\hbar \mathrm{c}}\left(\mathbb{A}_{j}(x)+\left[\partial_{j} \lambda(x)\right]\right)\right\} \cdot
\end{aligned}
$$

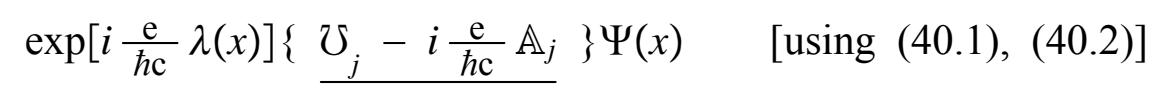

$$
\begin{aligned}
& =\exp \left[i \frac{\mathrm{e}}{\hbar \mathrm{c}} \lambda(x)\right]\left\{\underline { \{ \mho _ { j } - i \frac { \mathrm { e } } { \hbar \mathrm { c } } \mathbb { A } _ { j } \} } \cdot \left\{\underline{\left.\mho_{j}-i \frac{\mathrm{e}}{\hbar \mathrm{c}} \mathbb{A}_{j}\right\}} \Psi(x)\right.\right. \\
& \mathbb{D}_{j}^{\prime} \Psi^{\prime}(x)=\exp \left[i \frac{\mathrm{e}}{\hbar \mathrm{c}} \lambda(x)\right] \mathbb{D}_{j}^{2} \Psi(x)
\end{aligned}
$$

Then using (44) and (35), we have

$$
\begin{aligned}
\left\{-\frac{\hbar^{2}}{2 \mathrm{~m}}(\overrightarrow{\mathbb{D}})^{\prime 2}+e \phi\right\} \Psi^{\prime} & =\exp \left[i \frac{\mathrm{e}}{\hbar \mathrm{c}} \lambda(x)\right] \cdot\left\{-\frac{\hbar^{2}}{2 \mathrm{~m}}(\overrightarrow{\mathbb{D}})^{2}+e \phi\right\} \Psi \\
& =\exp \left[i \frac{\mathrm{e}}{\hbar \mathrm{c}} \lambda(x)\right] \cdot i \hbar \partial_{t} \Psi \\
i \hbar \partial_{t} \Psi^{\prime} & =i \hbar \partial_{t}\left\{\exp \left[i \frac{\mathrm{e}}{\hbar \mathrm{c}} \lambda(x)\right]\right\} \Psi=i \hbar \partial_{t} \Psi^{\prime}
\end{aligned}
$$

So the gauge invariance of Schrödinger equation in space (2) is demonstrated by the above expatiation.

\subsection{Phase Factor of Fractional Statistics}

Then focus our attentation on the phase factor of fractional statistics by using line integral on (42) for any closed counterclockwise loop $C(\varphi)$, encircling the origin, which is the most fascinating phenomenon of line path integral (67).

c) As a matter of convenience, in the next paragraph use $e=h=c=1$. Now we will consider with a special gauge vector potential $\overrightarrow{\mathbb{A}}$ of wave function $\Psi(x)$ in space (2) as

$$
\overrightarrow{\mathbb{A}}=\frac{1}{2 r \sin \theta} \vec{e}_{\varphi}
$$

we see $\overrightarrow{\mathbb{A}}$ is an irrotational field

$$
\begin{aligned}
\vec{\nabla} \times \overrightarrow{\mathbb{A}}= & \frac{1}{r \sin \theta}\left[\partial_{\theta}\left(\sin \theta \cdot \frac{1}{2 r \sin \theta}\right)-\partial_{\varphi}(0)\right] \vec{e}_{r} \\
& +\frac{1}{r}\left[\frac{1}{\sin \theta} \partial_{\varphi}(0)-\partial_{r}\left(r \cdot \frac{1}{2 r \sin \theta}\right)\right] \vec{e}_{\theta} \\
& +\frac{1}{r}\left[\partial_{r}(r \cdot 0)-\partial_{\theta}(0)\right] \vec{e}_{\varphi}(46) \\
\vec{\nabla} \times \overrightarrow{\mathbb{A}}= & 0
\end{aligned}
$$


In space (2), differential length is defined as

$$
\overrightarrow{D l}=\vec{e}_{r} d r+\vec{e}_{\theta} r \sin ^{4 m_{0}} \theta d \theta+\vec{e}_{\varphi} r \sin \theta h(\varphi) d \varphi
$$

Using (39), evaluating factor $\lambda(x)$ integral

$$
\begin{aligned}
\lambda(x) & =\int_{x_{0}[C]}^{x} \overrightarrow{\mathbb{A}} \cdot \overrightarrow{D l} \\
& =\oint_{C} \frac{1}{2 r \sin \theta} \vec{e}_{\varphi} \cdot \vec{e}_{\varphi} r \sin \theta \mathrm{h}(\varphi) d \varphi \\
& =\frac{1}{2} \oint_{C} \mathrm{~h}(\varphi) d \varphi=\frac{1}{2} \oint_{C} d \varphi\left\{\kappa+2 \eta \mathbb{T}_{1}(\varphi)\right\} \\
\lambda(x) & =\pi \kappa I_{0}=\pi\left(1+4 m_{0}\right) I_{0}=\pi(1+2 n) I_{0}
\end{aligned}
$$

(52) shows the contour integral of gauge vector $\overrightarrow{\mathbb{A}}$ is directly connected with the spin quantum numbers $n$ of particles of CSH! without any phenomenological postulation.

d) For clearer, resume physical units, gauge potential (48) turns to (54)

$$
\begin{aligned}
& \overrightarrow{\mathbb{A}}=\vec{e}_{\varphi} \frac{1}{2 r \sin \theta} \Phi \\
& \Phi=\frac{\hbar \mathrm{c}}{\mathrm{e}}=\Phi_{0} / 2 \pi
\end{aligned}
$$

where $\Phi_{0}$ is fundamental magnetic flux.

According to (37), the period of phase factor of wavefunction are written as

$$
\begin{aligned}
\exp \left[i \frac{\mathrm{e}}{\hbar \mathrm{c}} \lambda(x)\right] & =\exp \left[i \frac{\mathrm{e}}{\hbar \mathrm{c}} \int_{C(\varphi)}^{x} \overrightarrow{\mathbb{A}} \cdot \overrightarrow{D l}\right. \\
& =\exp \left[i \frac{\mathrm{e}}{\hbar \mathrm{c}} \Phi \int_{C(\varphi)}^{x} \vec{e}_{\varphi} \frac{1}{2 r \sin \theta} \cdot \overrightarrow{D l}\right.
\end{aligned}
$$
have

take $\Phi$ to be fundamental magnetic flux $\Phi_{0}$, and use (37),(39),(53),(57), we

$$
\begin{aligned}
\Psi(x) & \Rightarrow \Psi(x) \exp \left[i \oint_{C} \frac{1}{2 r \sin \theta} \vec{e}_{\varphi} \cdot \vec{e}_{\varphi} r \sin \theta \mathrm{h}(\varphi) d \varphi\right] \\
& =\Psi(x) \exp \left[i \pi(1+2 n) I_{0}\right]
\end{aligned}
$$


e) Using (59), make classfication of spin particles by quantun number $n$.

1) For Bosons:

$$
\begin{array}{r}
n=n_{+}, \text {are integers, such } 0,+1,+2,+3, \ldots, \\
\text { then phase are }+\pi,+3 \pi,+5 \pi,+7 \pi \ldots ; \\
n=n_{-}, \text {are integers, such } 0,-1,-2,-3, \ldots, \\
\text { then phase are }+\pi,-\pi,-3 \pi,-5 \pi \ldots .
\end{array}
$$

2) For Fermions:

$$
\begin{array}{r}
n=n_{+}, \text {are half-integers, such }+1 / 2,+3 / 2,+5 / 2,+7 / 2 \ldots, \\
\text { then phase are }+2 \pi,+4 \pi,+6 \pi,+8 \pi \ldots ; \\
n=n_{-}, \text {are half-integers, such }-1 / 2,-3 / 2,-5 / 2,-7 / 2, \ldots, \\
\text { then phase are }+0 \pi,-2 \pi,-4 \pi,-6 \pi \ldots .
\end{array}
$$

We see all Bosons lie at the negative real coordinate axis and all Fermions lie at the positive real coordinate axis

3) For TKP: $n_{+}$and $n_{-}$are neither integers nor half-integers.

$$
\begin{array}{ll}
n=n_{+}, \text {are }+1 / 3,+2 / 3, \ldots, & \text { then phase are }+5 \pi / 3,+7 \pi / 3(+\pi / 3), \ldots ; \\
n=n_{-}, \text {are }-1 / 3,-2 / 3, \ldots, & \text { then phase are }+\pi / 3,-\pi / 3, \ldots \\
n=n_{+}=+1 / 4, & \text { then phase is }+3 \pi / 2 ; \\
n=n_{-}=-1 / 4, & \text { then phase is }+\pi / 2 .
\end{array}
$$

We can choose different vector potential $\overrightarrow{\mathbb{A}}$, for different physical pictures in space (2), then we will have different represents of (59) or (37).

\subsection{The Third Kind of Particles, TKP are not Anyons cite: [5]}

1) The generators of Anyons do not satisfy the commutation rules of angular momentum, so Anyons are not real spin particles; The generators of TKP obey angular momentum commutation relations, they are true spin particles.

2) Anyons are classified according to braind group $B_{N}$; TKP are the extension of groups $S O(3)$ and $S U(2)$.

3) Anyons exist only in two dimensional system; TKP are exist in three dimensional system.

4) Anyons do not depend on space metric, that be formulated from topological quantum field theory; The physical concepts of TKP arise from angular momentum, which are tightly connected with the three-dimensional space construction. 


\section{Conclusion}

Now back to chapter $\mathbf{0}$, in conventional quantum mechanics, the eigenvalues $\mu$ of total square operator $J^{2}$ of spin angular momentum are expressed by $(0-23)$ and the representations of matrix dimensionality $D$ of spin angular momentum are related to the values of the spin particles as shown with formula (0-24).

\section{Note}

It is seen, that due to the eigenvalues $\lambda$ of the third component matrix $J_{3}$ could extend to $\pm \infty$,

formulae (0-23) and (0-24) are untenable !

as follow

1) For an example, in conventional quantum mechanics, $\lambda_{\max }$ is integer and half-integer in the dimensionality formula $D(0-24)$. We see, the less the value of $\lambda_{\max }$, the small the matrix dimensionality of spin angular momentum. From spin $1 \hbar$, to spin $\hbar / 2$, to spin $0 \hbar$, the dimensionality is 3 , to, 2 , to 1 .

What will happen ? if $\lambda_{\max }$ continues to decrease in interval $(\hbar / 2,0 \hbar)$.

If $\lambda_{\max }=\operatorname{spin} \hbar / 3, \quad$ spin $\hbar / 4, \quad$ spin $\hbar / 5, \quad$ spin $\hbar / 6, \ldots$

Then $D=2 \lambda_{\max }+1=2 / 3+1,2 / 4+1,2 / 5+1,2 / 6+1, \ldots$ $=5 / 3,6 / 4,7 / 5,8 / 6, \ldots$ dimensionality $D$ is fractional !

This means that if formula (0-24) were still valid, you should construct so-called fractional-matrix ! which is imcompatible with the skeleton frame of the present math, at least. So there are only two kinds of spin particles in conventional quantum mechanics.

Whereas, after the exposition of chapter $\mathbf{5}$ and chapter $\mathbf{6}$, and later three examples of typical spin particles of CSP ( chapters 7, 8,9), it is shown that in the system of Chaos Spin Hierarchy, the dimensionalities $D$ of spin $0 \hbar \hbar / 2, \hbar / 3$ extend to infinite!

Actually, in Chaos Spin Hierarchy (in chapter 6), all the members of spin angular momentum, $\xi_{1, n}^{( \pm)}(6-1),(6-5), \xi_{2, n}^{( \pm)}(6-2),(6-6), \xi_{3, n}^{( \pm)}(6-3),(6-7)$ and $\left(\xi_{n}^{( \pm)}\right)^{2}$ $(6-4),(6-8)$ are expressed by infinite matrices, which are indenpendent of the spin values $\lambda$ of the particles.

Further the axiom (0-24) should be abandoned. If we want to still hold down the $(0-24)$, after all, $D=2 \lambda_{\max }+1= \pm \infty$, in this sense, that seems to somewhat "reasonable". 
2) When $\lambda$ extends to $\lambda=\lambda_{\max }= \pm \infty$, further formula (0-23) that concerns about the eigenvalue $\mu$ of total square operator $J^{2}$ of spin angular momentum, is invalid too.

Actually, in Chaos Spin Hierarchy, ther are two dimensionality formula (6-4) and (6-8) which highly resemble $(0-23)$

$$
\mu=\frac{\lambda_{\max }}{(0-23)}\left(\underline{\lambda_{\max }}+1\right)=k / 2(k / 2+1) \Rightarrow n(n \pm 1)=n_{ \pm}\left(n_{ \pm} \pm 1\right)=\left(\xi_{n}^{( \pm)}\right)^{2}
$$

But their derivations are quite different:

a) In (0-23), the eigenvalue $\mu$ of total square operator $J^{2}$ is symboled by $k / 2(k / 2+1)$ that be derived from $\underline{\lambda}_{\max }\left(\underline{\lambda}_{\max }+1\right)$. Here $\underline{\lambda_{\max }}$ is the maximum of $J_{3}$ in conventional mechanics, corresponds to quantum number $m$.

b) (6-4), (6-8) represented by quantum number $n_{+}=+2 m_{0}(4-7), n_{-}=-2 m_{0}(4-8)$. Here $2 m_{0}$ is a parameter that depicts the curvature of space $\alpha=\mathrm{h}(\varphi) \mathrm{g}(\theta)(2-5)$, which initially appears in the eigenvalue expressions $m \pm 2 m_{0}$ of non-Hermitian angular momentum $\mathbb{L}_{3}$.

This paper shows how Non-Hermitian angular momentum. comes to what TKP is today

\section{Chaos Spin Hierarchy (CSH)}

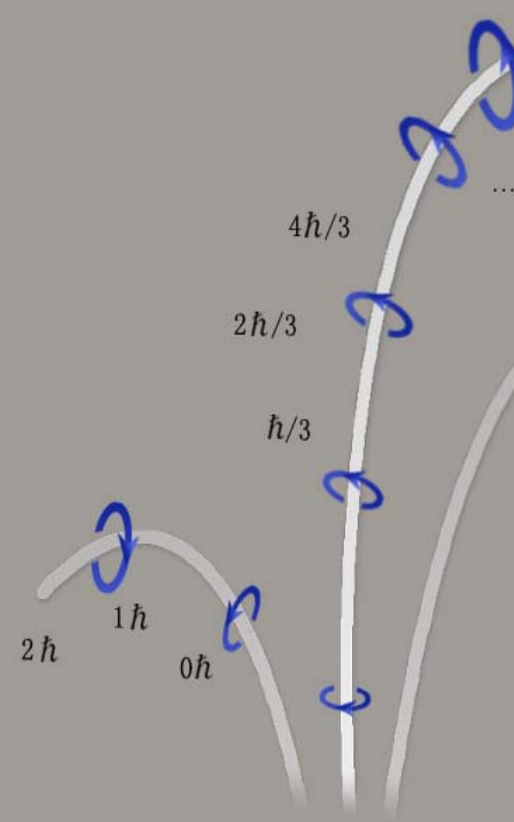

TKP:

Formulae

$\xi_{j, \mathrm{n}}^{(+)}:(6-1),(6-2),(6-3), \quad(6-4)$

$\xi_{\mathrm{j}, \mathrm{n}}^{(-)}:(6-5),(6-6),(6-7), \quad(6-8)$ 
Author places some hope on the adoption above, to give a good deal of enlightenment for both students and researchers, and wish the formulations of TKP is compatible with the axiomatic of quantum mechanics known as yet, further an idea in the uses of future physics.

In this paper, some fundamental research, which results in non-Hermitian angular momentum (both orbit and spin) of author's work for past years, are given cite: [3],[4].

TKP methodology is applied to Energy Harmonic Oscillator Hierarchy (EHOH) and infinite dimensional matrices of Lorentz Group, much of which have never been published in public journals before cite: [3].

This article mainly comes from author's English-Lectures that designed to serve the needs of workshop and seminar in China.

\section{ACKNOWLEDGEMENT}

Author thanks Yong-Shi Wu, Professor of University of Utah USA, for his helps and encouragements

\section{REFERENCES}

[1] Bender C M, Boettcher S, F.G Scholtz, H.B Geyer, F. Hahne, A.Mostafazadeh, C. Figueira de Morisson Faria, Wu Jund and A.Fring, H.F.Jones, M.Znojil.

[2] A.Mostafazadeh 2002 J.Math.Phys. 43205 2814, 3944

[3] ShaoXu Ren, Advanced Non-Euclidean Quantum Mechanics ISBN 978-7-80703-585-4 (2006);

Frontier Science ISSN 1673-8128. vol 2, (2008); vol 5, (2011);

The Third Kind of Particles ISBN 978-7-900500-91-5 (2011);

The Third Kind of Particles ISBN 978-988-15598-9-0 (2012);

The Third Kind of Particles ISBN 978-3-659-17892-4 (2012);

Faster Than Velocity Of Light ISBN 978-988-12266-2-4 (2013) .

[4] ShaoXu Ren, Scientific Programm of the ICAP 2006-20th International Conference on Atom Physics, Innsbruck, Austria.

[5] Leinaas, J.M.; J. Myrheim (11 January 1977). On the theory of identical particles. Il Nuovo Cimento B 37 (1): 1-23.

Wilczek, Frank (4 October 1982). Quantum Mechanics of Fractional-Spin Particles. Physical Review Letters 49 (14): 957-959. 


\section{Appendix: Infinitesimal Rotation of TKP}

A1) Infinitesimal Rotation of Particle of Spin $0 h$ CSH

Infinitesimal rotation operator $\mathbb{W}_{1,+0}=1 / \sqrt{2}$.

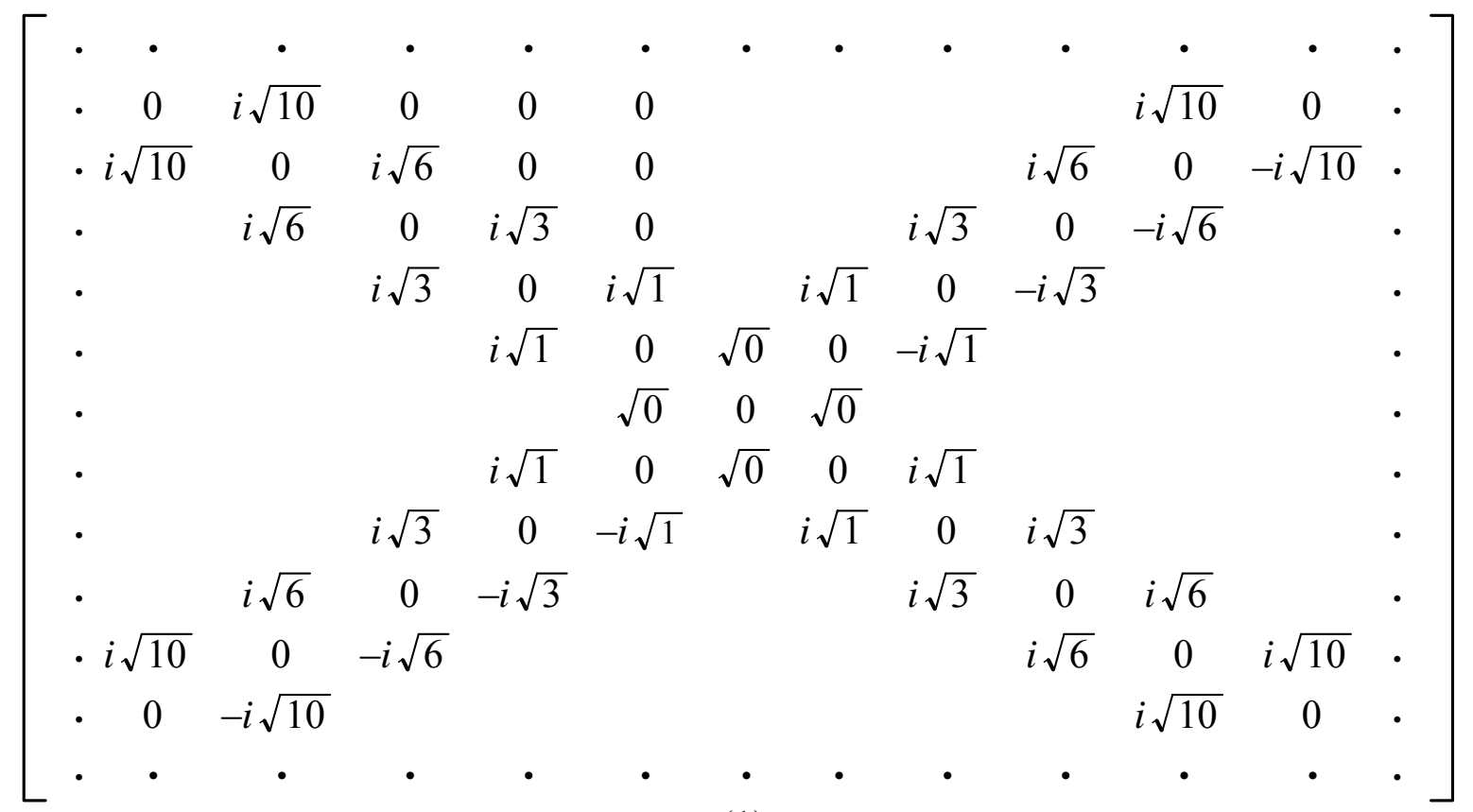

(1)

Infinitesimal rotation operator $\mathbb{W}_{2,+0}=1 / \sqrt{2} \cdot$

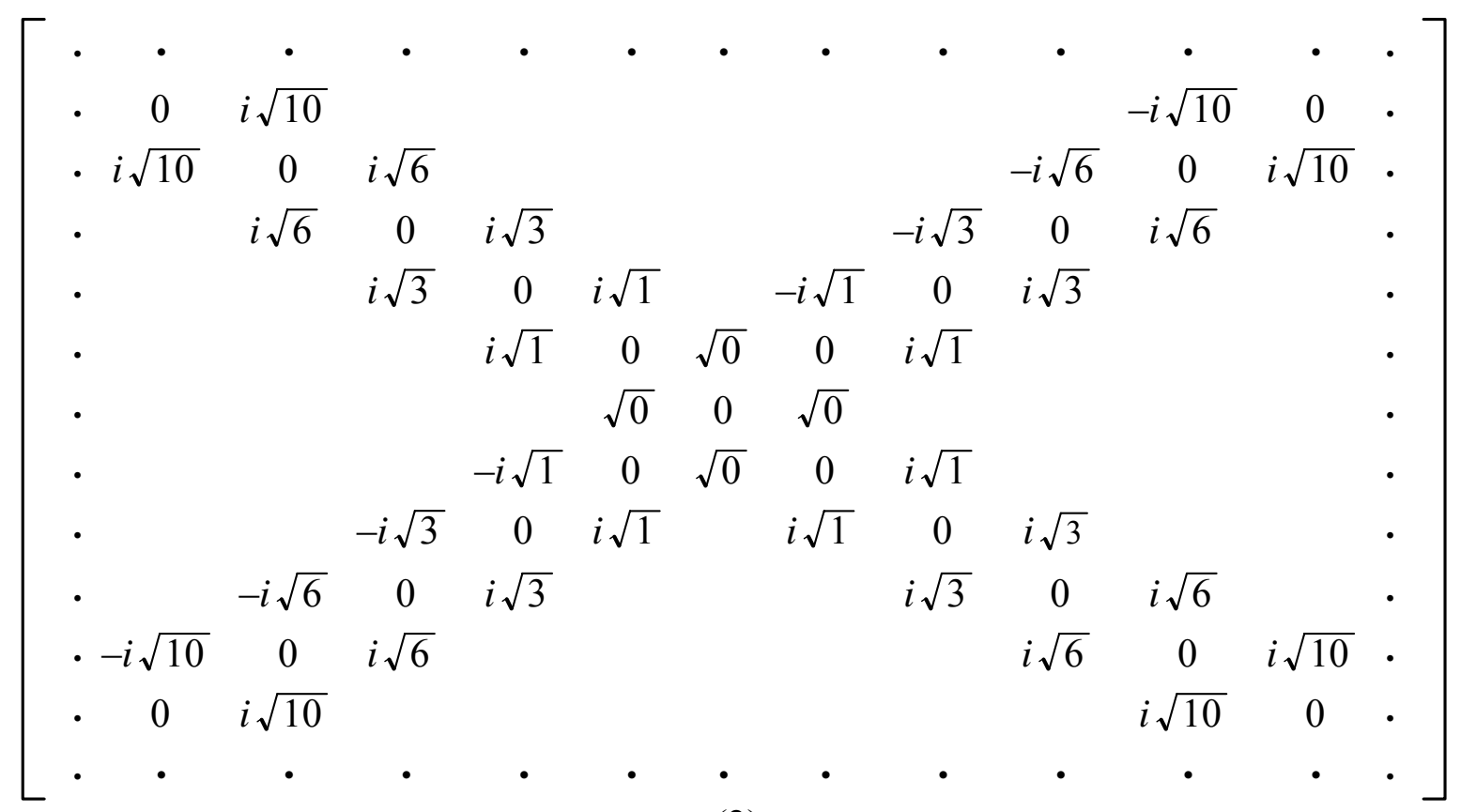

(2) 
A2) Infinitesimal Rotation of Particle of Spin $1 \hbar$ CSH cite: [3]

Infinitesimal rotation operator $\mathbb{W}_{1,+1}=1 / \sqrt{2}$.

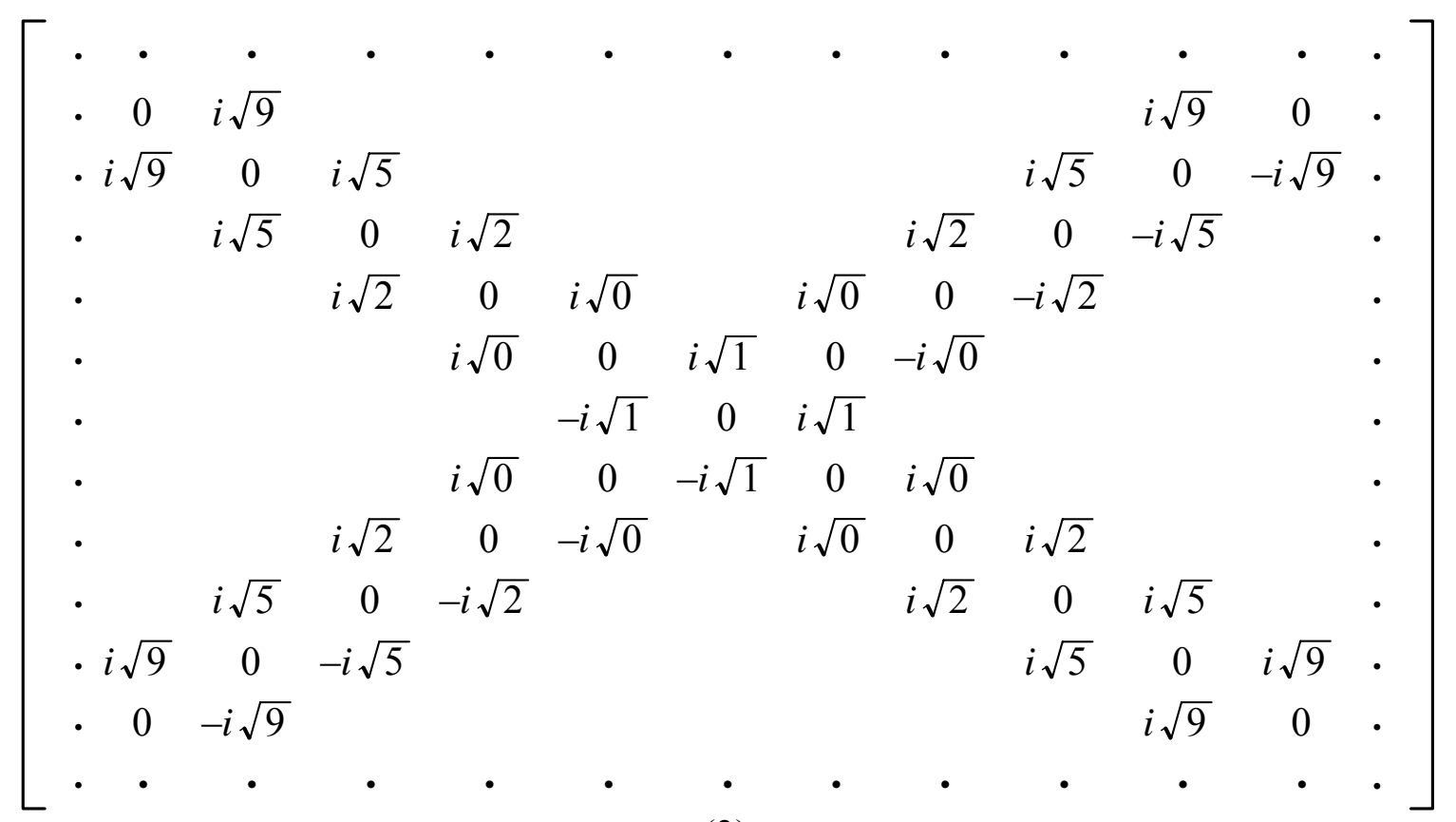

(3)

Infinitesimal rotation operator $\mathbb{W}_{2,+1}=1 / \sqrt{2}$.

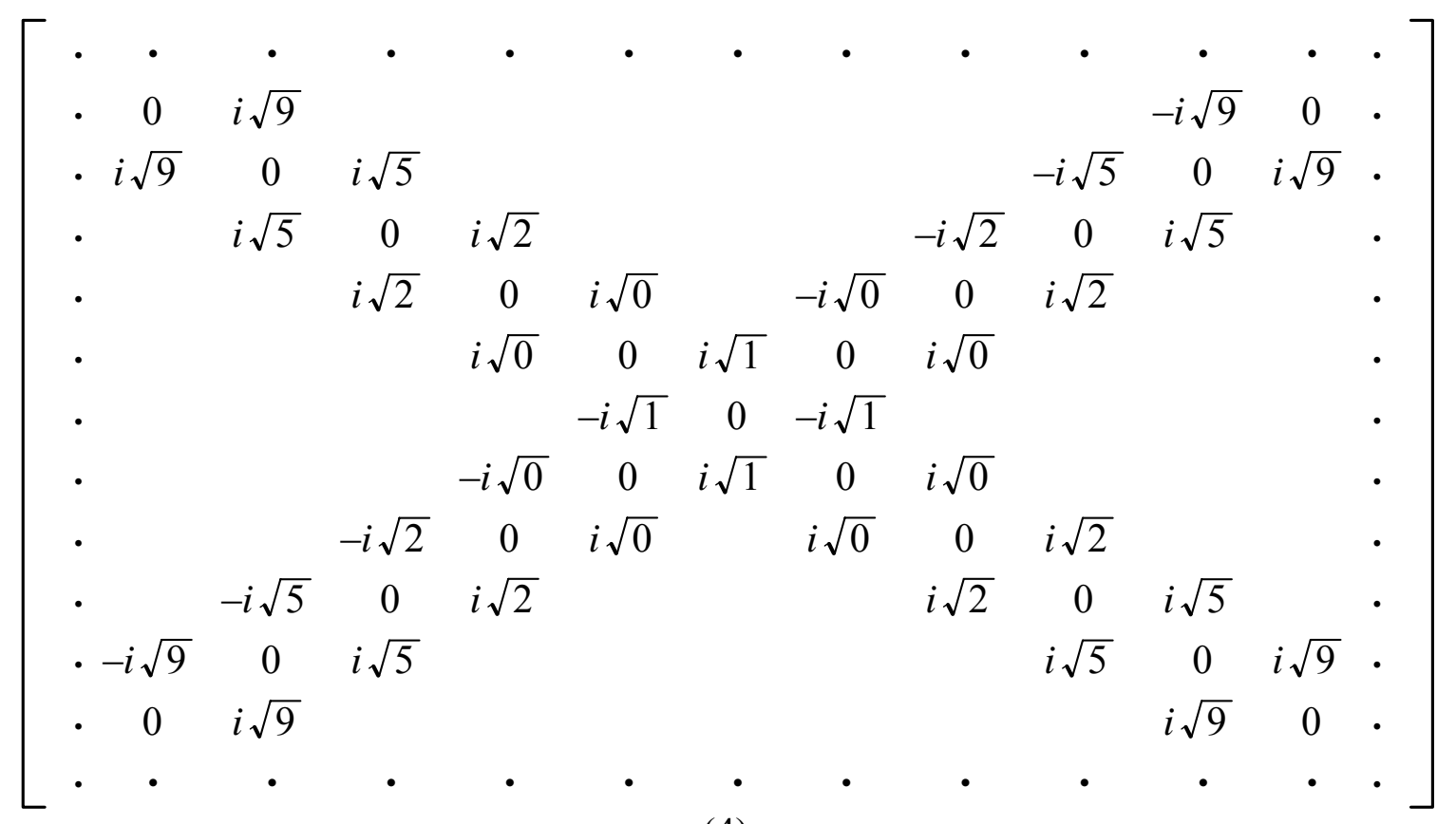

(4) 
A3) Particle of Spin 0 $\hbar$ CSH and Particle of Spin $1 \hbar$ CSH have the same math figure in their third Infinitesimal Rotation operators, that is

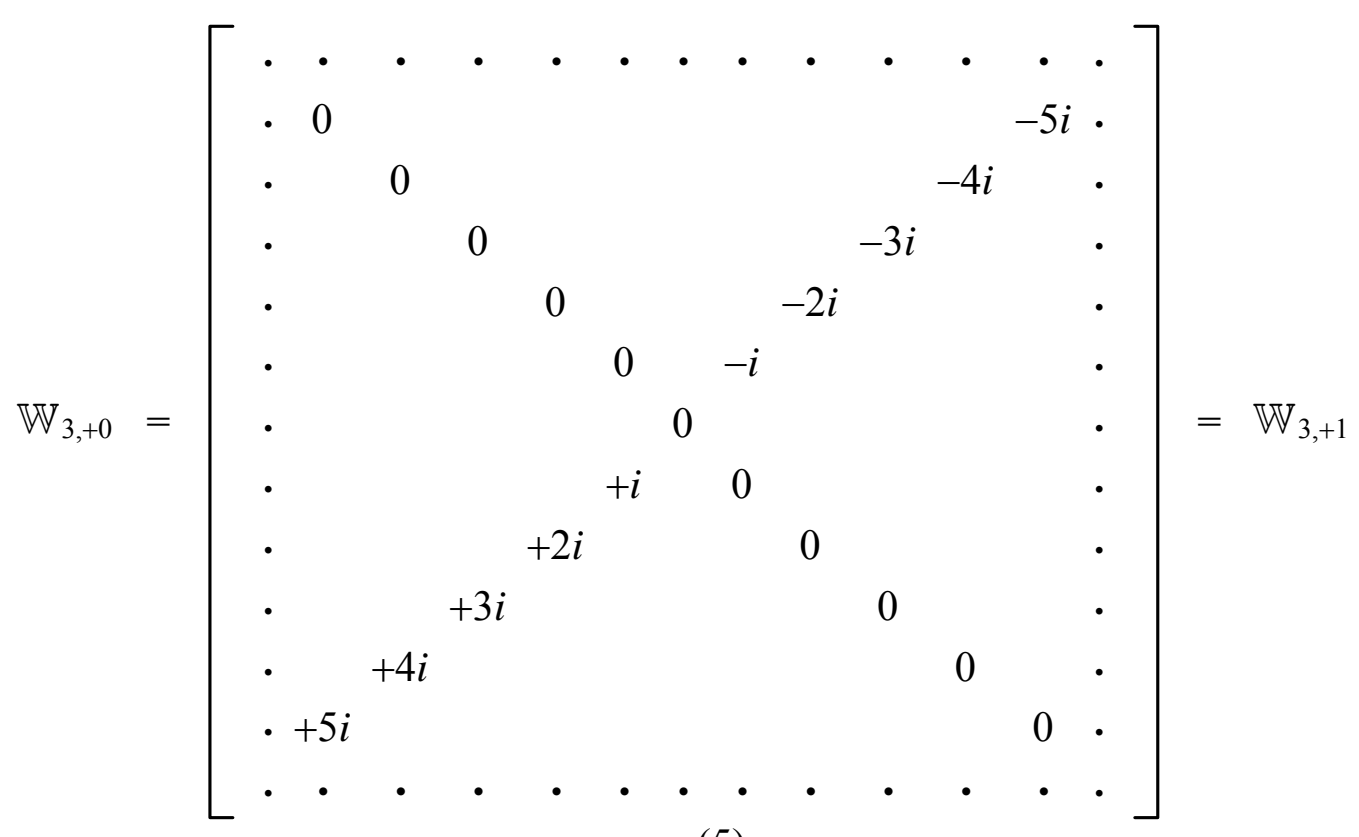

(5)

Operators $\mathbb{W}_{j,+n}$ obey angular momentum commutation rules

$$
\mathbb{W}_{j,+n} \mathbb{W}_{k,+n}-\mathbb{W}_{k,+n} \mathbb{W}_{j,+n}=i \hbar \mathbb{W}_{l,+n}
$$

Here space indexes $j, k, l=1,2,3$ are circulative;

particle spin quantum numbers $n=0,1$

A4) Operators $S_{j}, F_{j}, W_{j}$ are $3 \times 3$ matrices.

$$
\begin{aligned}
& S_{1}=\left[\begin{array}{ccc}
0 & 0 & 0 \\
0 & 0 & -i \\
0 & i & 0
\end{array}\right], \quad S_{2}=\left[\begin{array}{ccc}
0 & 0 & i \\
0 & 0 & 0 \\
-i & 0 & 0
\end{array}\right], \quad S_{3}=\left[\begin{array}{ccc}
0 & -i & 0 \\
+i & 0 & 0 \\
0 & 0 & 0
\end{array}\right] \\
& F_{1}=\left[\begin{array}{ccc}
0 & i & 0 \\
-i & 0 & 0 \\
0 & 0 & 0
\end{array}\right], \quad F_{2}=\left[\begin{array}{ccc}
0 & 0 & 0 \\
0 & 0 & -i \\
0 & i & 0
\end{array}\right], \quad F_{3}=\left[\begin{array}{ccc}
0 & 0 & -i \\
0 & 0 & 0 \\
i & 0 & 0
\end{array}\right] \\
& S_{j} S_{k}-S_{k} S_{j}=i \hbar S_{l} \\
& F_{j} F_{k}-F_{k} F_{j}=i \hbar F_{l} \\
& W_{1}=\frac{1}{\sqrt{2}}\left[\begin{array}{ccc}
0 & i & 0 \\
-i & 0 & i \\
0 & -i & 0
\end{array}\right], \quad W_{2}=\frac{1}{\sqrt{2}}\left[\begin{array}{ccc}
0 & i & 0 \\
-i & 0 & -i \\
0 & i & 0
\end{array}\right], \quad W_{3}=\left[\begin{array}{ccc}
0 & 0 & -i \\
0 & 0 & 0 \\
i & 0 & 0
\end{array}\right] \\
& W_{j} W_{k}-W_{k} W_{j}=i \hbar W_{l}
\end{aligned}
$$


In case of Spin $1 \hbar \mathbf{C S H}$, we select $W_{j}$ as the matrix center parts of $\mathbb{W}_{j,+1}$, due to the most symmetrical design of the math harmony of Infinitesimal rotation of TKP. Operators $F_{j}, W_{j}$ will be chosen as the center of $\mathbb{W}_{j,+n}$ in other physical pictures. 
Scientific Research Publishing (SCIRP) is one of the largest Open Access journal publishers. It is currently publishing more than 200 open access, online, peer-reviewed journals covering a wide range of academic disciplines. SCIRP serves the worldwide academic communities and contributes to the progress and application of science with its publication.

Other selected journals from SCIRP are listed as below. Submit your manuscript to us via either submit@scirp.org or Online Submission Portal.
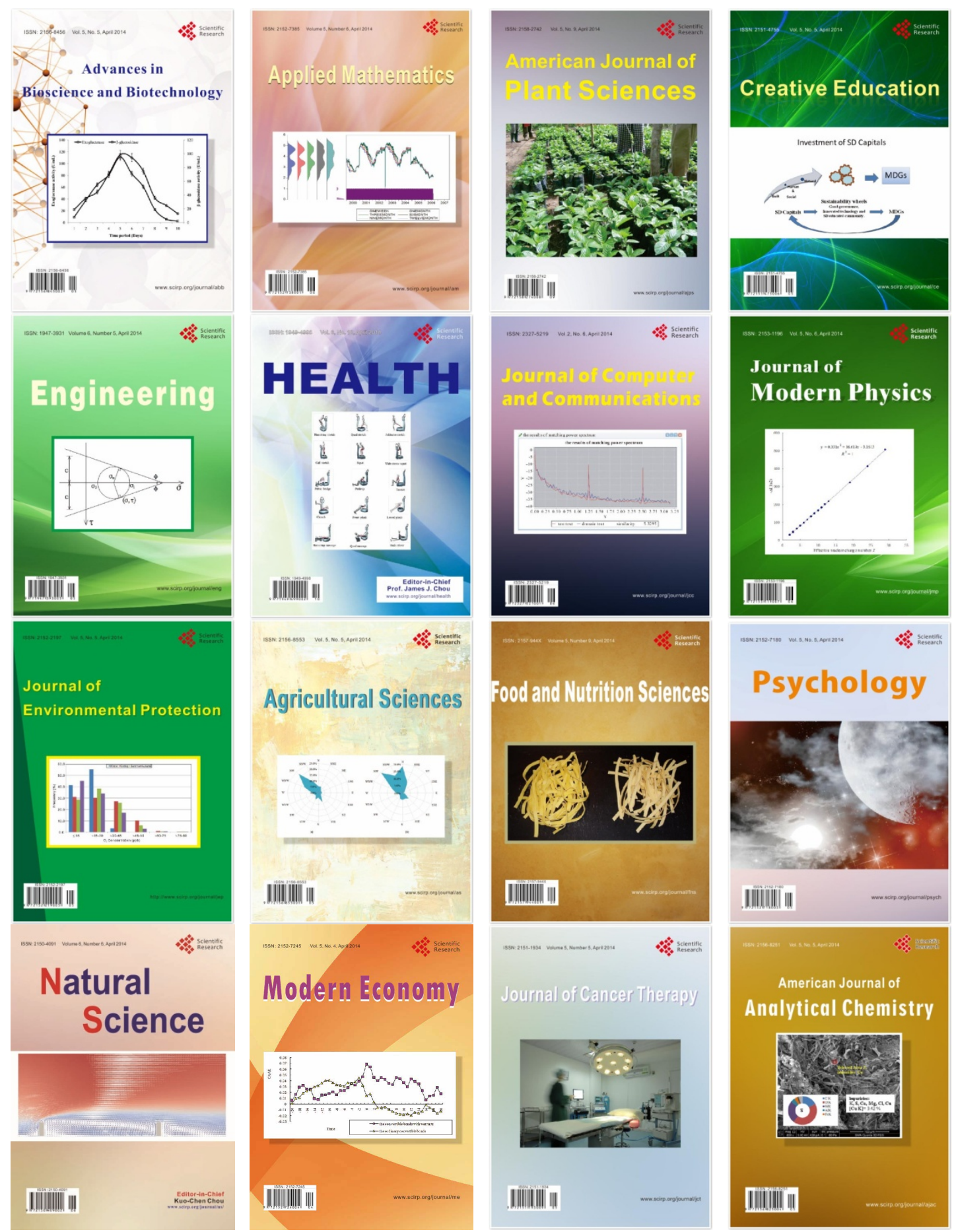